\title{
1997 INEEL National Emission Standard for Hazardous Air Pollutants - Radionuclides
}

\section{Annual Report June 1998}
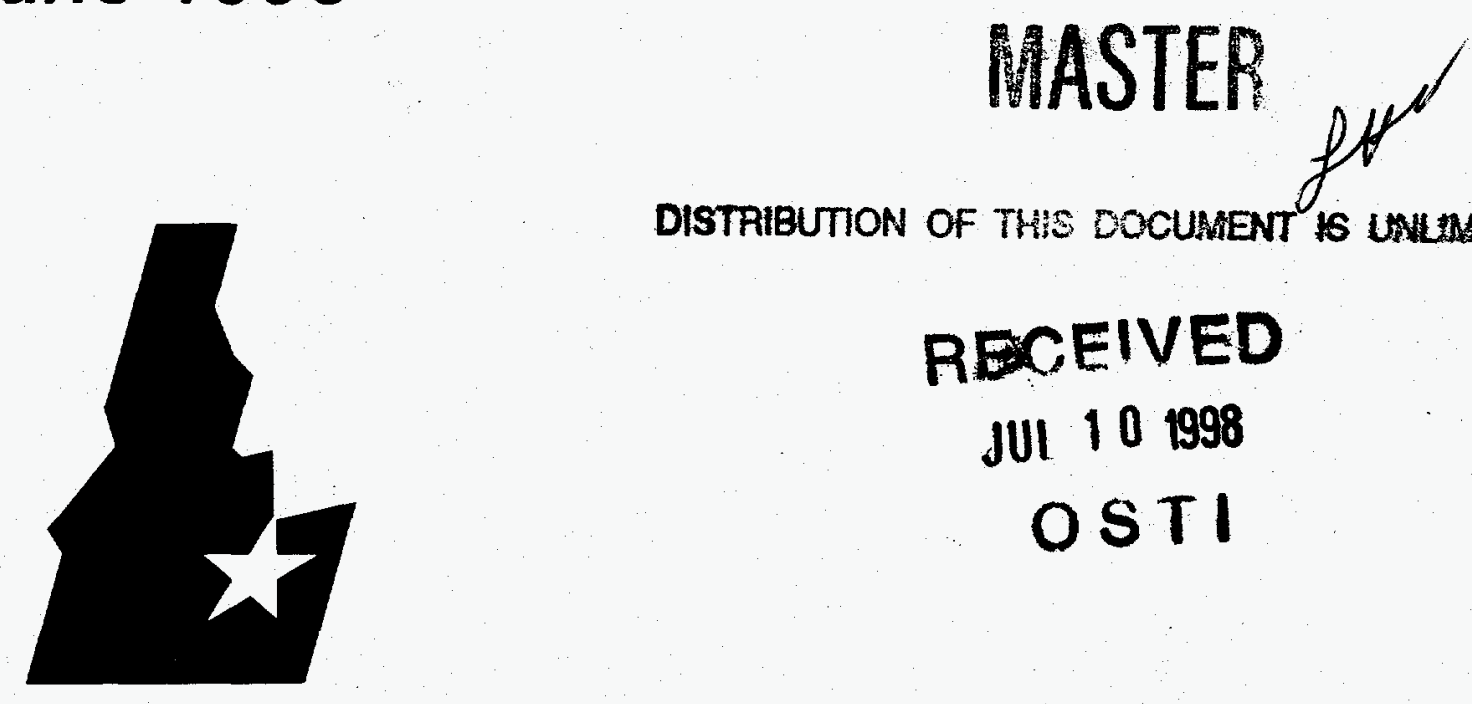

DISTAIBUTION OF THSS DOCUMENT IS UNLMMTES

$$
\text { RECEIVED }
$$

JUI 101998

OSTI

Idaho National Engineering Laboratory

\section{U.S. Department of Energy $\cdot$ Idaho Operations Office}

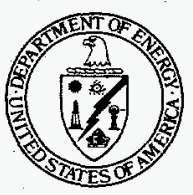




\section{Idaho National}

Engineering and Environmental Laboratory

(INEEL)

National Emission Standards

for Hazardous Air Pollutants

(NESHAPs) - Radionuclides

Annual Report

June 1998

U.S. Department of Energy

Idaho Operations Office 


\section{DISCLAIMER}

This report was prepared as an account of work sponsored by an agency of the United States Government. Neither the United States Government nor any agency thereof, nor any of their employees, makes any warranty, express or implied, or assumes any legal liability or responsibility for the accuracy, completeness, or usefulness of any information, apparatus, product, or process disclosed, or represents that its use would not infringe privately owned rights. Reference herein to any specific commercial product, process, or service by trade name, trademark, manufacturer, or otherwise does not necessarily constitute or imply its endorsement, recommendation, or favoring by the United States Government or any agency thereof. The views and opinions of authors expressed herein do not necessarily state or reflect those of the United States Government or any agency thereof. 


\section{DISCLAIMER}

Portions of this document may be illegible electronic image products. Images are produced from the best available original document. 


\section{U. S. DEPARTMENT OF ENERGY \\ RADIONUCLIDE AIR EMISSIONS ANNUAL REPORT \\ (under Subpart H of 40 CFR 61) \\ CALENDAR YEAR 1997}

Site Name: Idaho National Engineering and Environmental Laboratory

\section{Operations Office Information}

Office:

Address:

Contact:

Phone:
Department of Energy - Idaho Operations Office

785 DOE Place

Idaho Falls, Idaho 83401

John Medema

(208) $526-1407$

Site Information:

For information regarding facilities on the INEEL, contact the Idaho Operations Office above. 



\section{TABLE OF CONTENTS}

ACRONYMS vii

EXECUTIVE SUMMARY ix

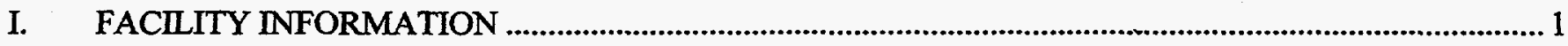

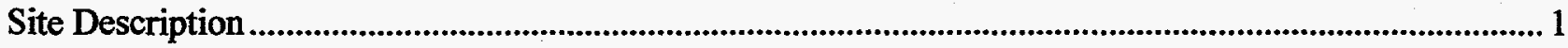

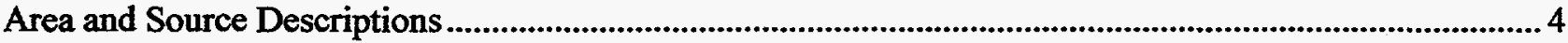

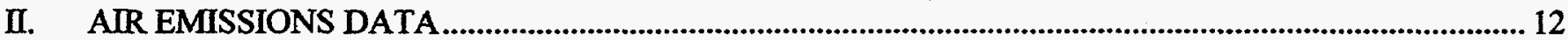

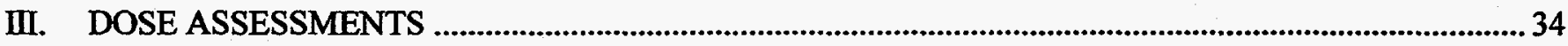

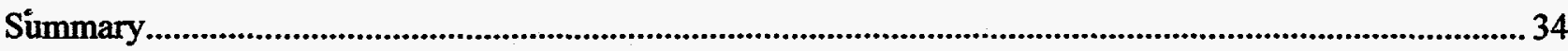

Description of Dose Model and Summary of Input Parameters ..................................................................38

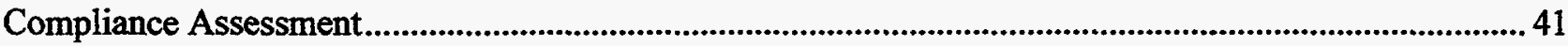

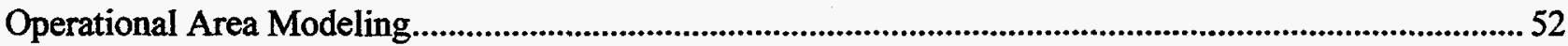

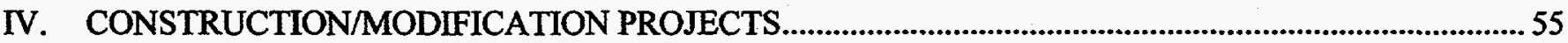

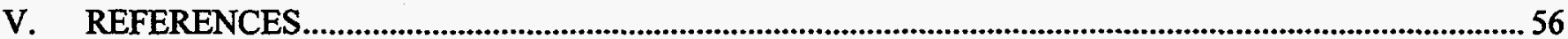

\section{APPENDICES}

A. Naval Reactors Facility Radionuclide Air Emissions Report ….......................................................

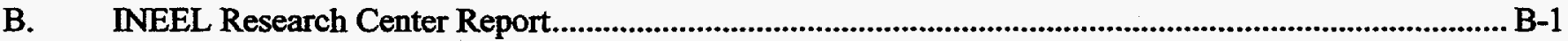

C. 1997 Meteorology Data For CAP-88 Computer Code .............................................................................. C-1

D. Input Parameter Values For CAP-88 Computer Code .............................................................................. D-1

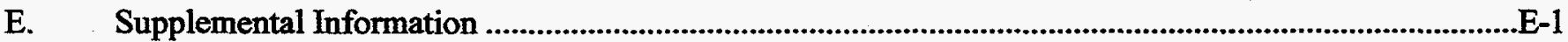

\section{FIGURES}

1. Idaho National Engineering And Environmental Laboratory And Major Facilities...................................2

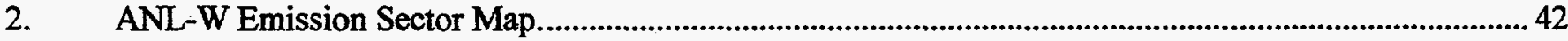

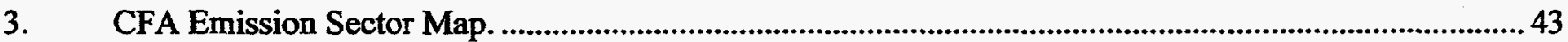

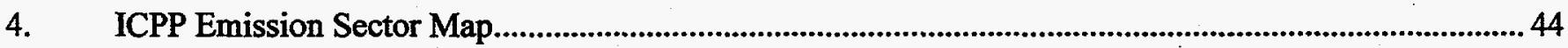

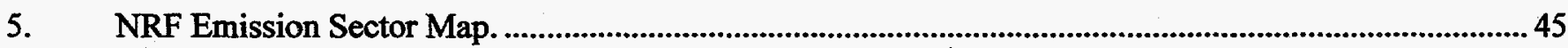

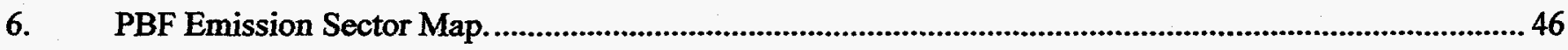

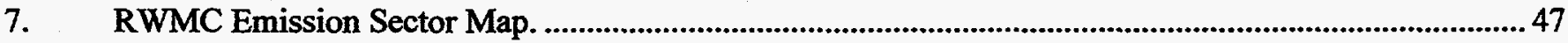

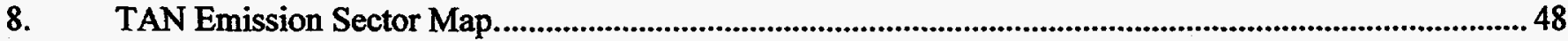

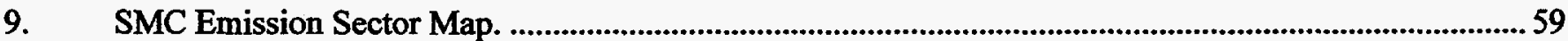

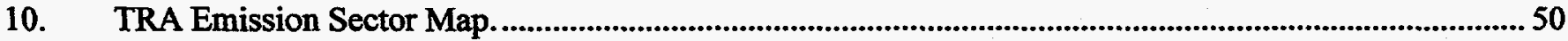




\section{TABLES}

II-1 . ANL-W Radiological Air Emission Sources Which Had Emissions In CY 1997 ................................... 14

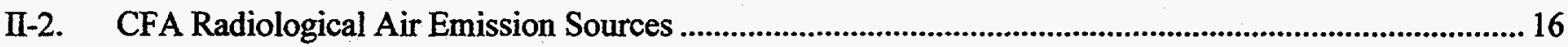

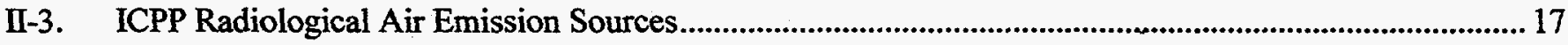

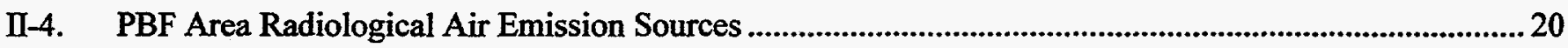

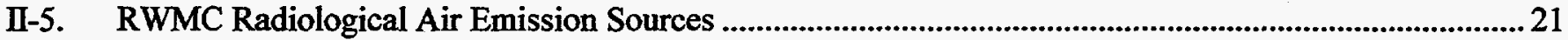

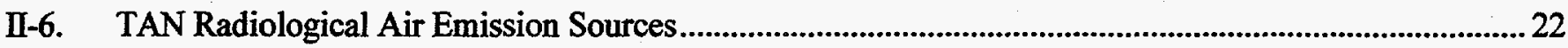

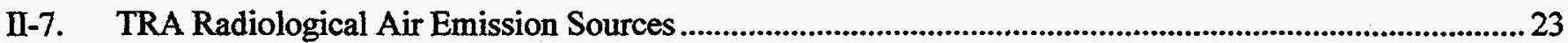

II-8. Point Source Radionuclides (Continuously Compliance Monitored Sources) From INEEL

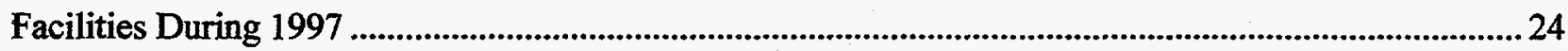

II-9. Point Source Radionuclides (All Other Release Points) From The INEEL Facilities During 1997 ......... 26

II-10. Non-Point Source Radionuclides (Diffuse Emission Sources) From The INEEL Facilities

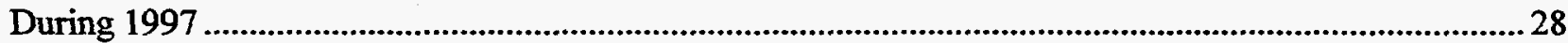

III-1. Summary of 1997 Effective Dose Equivalents To The MEI From Continuously Compliance Monitored

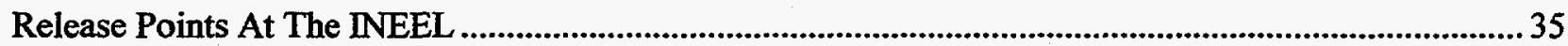

III-2. Summary of 1997 Effective Dose Equivalents From Other Release Points At The INEEL ...................36

III-3. Summary of 1997 Effective Dose Equivalents From Diffuse Sources At The INEEL ............................. 37

III-4. Sources Of Wind Data For 1997 CAP-88 Atmospheric Dispersion Modeling Of Releases

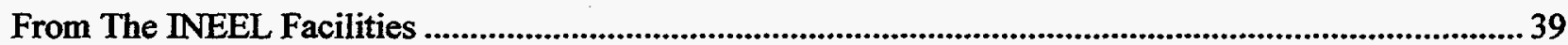

III-5. INEEL Stack Data for Releases Modeled As Stack Releases ................................................................40

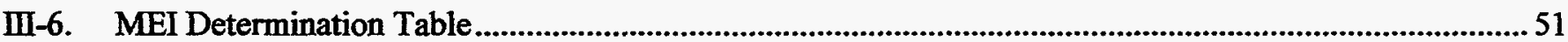

\section{APPENDICES TABLES}

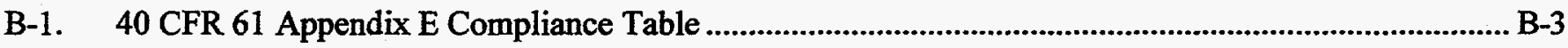

C-1. STAR File Data Used For ANL Ground-level releases ....................................................................

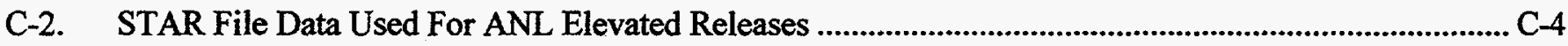

C-3. STAR File Data Used For CFA (and RWMC) Ground-level releases .............................................. C-5

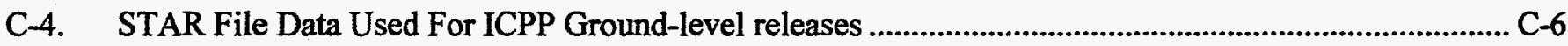

C-5. STAR File Data Used For ICPP Elevated Releases ...........................................................................

C-6. STAR File Data Used For NRF Ground-level releases ........................................................................

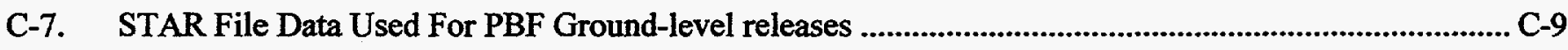

C-8. STAR File Data Used For TAN Ground-level releases .....................................................................

C-9. STAR File Data Used For TAN Elevated Releases ...........................................................................

C-10. STAR File Data Used For TRA Ground Level and Elevated Releases ................................................ C-12

D-1. Input Parameter Values For CAP-88 Computer Code........................................................................... 


\section{ACRONYMS}

$\begin{array}{ll}\text { ANL } & \text { Argonne National Laboratory } \\ \text { ANL-W } & \text { Argonne National Laboratory - West } \\ \text { ATR } & \text { Advanced Test Reactor } \\ \text { CAM } & \text { constant air monitor } \\ \text { CEDE } & \text { committed effective dose equivalent } \\ \text { CFA } & \text { Central Facilities Area } \\ \text { CFR } & \text { Code of Federal Regulations } \\ \text { CY } & \text { calendar year } \\ \text { DOE } & \text { Department of Energy } \\ \text { DOE-CH } & \text { Department of Energy, Chicago Operations Office } \\ \text { DOE-ID } & \text { Department of Energy, Idaho Operations Office } \\ \text { DU } & \text { depleted uranium } \\ \text { DVF } & \text { Drum Venting Facility } \\ \text { EBR-II } & \text { Experimental Breeder Reactor-II } \\ \text { ECF } & \text { Expended Core Facility } \\ \text { EDE } & \text { effective dose equivalent } \\ \text { EPA } & \text { Environmental Protection Agency } \\ \text { FASB } & \text { Fuel Assembly and Storage Building } \\ \text { FAST } & \text { Fluorinel and Storage Facility } \\ \text { FCF } & \text { Fuel Conditioning Facility } \\ \text { FHU } & \text { fuel handling unit } \\ \text { FMF } & \text { Fuel Manufacturing Facility } \\ \text { Foundation } & \text { Environmental Science and Research Foundation } \\ \text { HEPA } & \text { high-efficiency particulate air } \\ \text { HEU } & \text { Highly Enriched Uranium } \\ \text { HFEF } & \text { Hot Fuel Examination Facility } \\ \text { HLLWE } & \text { High-Level Liquid Waste Evaporator } \\ \text { HLW } & \text { high-level waste } \\ \text { HP } & \text { health physics } \\ \text { HPIL } & \text { Health Physics Instrumentation Laboratory } \\ \text { HRF } & \text { heat recovery fan } \\ \text { HVAC } & \text { heating, ventilating, and air conditioning } \\ \text { ICPP } & \text { Idaho Chemical Processing Plant } \\ \text { NNEEL } & \text { Idaho National Engineering and Environmental Laboratory } \\ \text { IRC } & \text { INEEL Research Center } \\ \text { L\&O } & \text { Laboratory and Office } \\ \text { LET\&D } & \text { Liquid Effluent Treatment and Disposal } \\ \text { LMTTCO } & \text { Lockheed Martin Idaho Technologies Company } \\ \text { MDF } & \text { Materials Development Facility } \\ \text { MEI } & \text { maximally exposed individual } \\ \text { MTR } & \text { Material Test Reactor } \\ \text { NCRP } & \text { National Council on Radiation Protection } \\ \text { NDA } & \text { nondestructive assay } \\ \text { NESHAPs } & \text { National Emission Standards for Hazardous Air Pollutants }\end{array}$




\section{ACRONYMS (continued)}

\begin{tabular}{|c|c|}
\hline NOAA & National Oceanic and Atmospheric Administration \\
\hline NRC & Nuclear Regulatory Commission \\
\hline NRF & Naval Reactors Facility \\
\hline NWCF & New Waste Calcining Facility \\
\hline PBF & Power Burst Facility \\
\hline PEW & Process Equipment Waste \\
\hline PHP & Plasma Hearth Process \\
\hline PNR & Pittsburgh Naval Reactors \\
\hline PRF & Process Reclamation Facility \\
\hline QA & quality assurance \\
\hline PRF & Process Reclamation Facility \\
\hline RAL & Remote Analytical Laboratory \\
\hline RESL & Radiological and Environmental Sciences Laboratory \\
\hline RLWTF & Radioactive Liquid Waste Treatment Facility \\
\hline RPSSA & Radioactive Parts Security Storage Area \\
\hline RWMC & Radioactive Waste Management Complex \\
\hline RWMIS & Radioactive Waste Management Information System \\
\hline SCMS & Sodium Components Maintenance Shop \\
\hline SDA & Subsurface Disposal Area \\
\hline SMC & Specific Manufacturing Capability \\
\hline SNF & spent nuclear fuel \\
\hline STAR & Stability wind rose (STability ARray file) \\
\hline SWEPP & Stored Waste Examination Pilot Plant \\
\hline TAN & Test Area North \\
\hline TRA & Test Reactor Area \\
\hline TREAT & Transient Reactor Test Facility \\
\hline TSA & Transuranic Storage Area \\
\hline TSF & Technical Support Facility \\
\hline WEC & Westinghouse Electric Company \\
\hline WERF & Waste Experimental Reduction Facility \\
\hline WIPP & Waste Isolation Pilot Plant \\
\hline WROC & Waste Reduction Operations Complex \\
\hline WSF & Waste Storage Facility \\
\hline ZPPR & Zero Power Physics Reactor \\
\hline
\end{tabular}




\section{EXECUTIVE SUMMARY}

Under Section 61.94 of Title 40, Code of Federal Regulations (CFR), Part 61, Subpart H, "National Emission Standards for Emissions of Radionuclides Other Than Radon From Department of Energy Facilities," each Department of Energy (DOE) facility must submit an annual report documenting compliance. This report addresses the Section 61.94 reporting requirements for operations at the Idaho National Engineering and Environmental Laboratory (INEEL) for calendar year (CY) 1997. The Idaho Operations Office of the DOE is the primary contact concerning compliance with the National Emission Standards for Hazardous Air Pollutants (NESHAPs) at the INEEL.

For CY 1997, airborne radionuclide emissions from the INEEL operations were calculated to result in a maximum individual dose to a member of the public of 2.77E-02 mrem (2.77E-07 Sievert). This effective dose equivalent (EDE) is well below the 40 CFR 61, Subpart $H$, regulatory standard of 10 mrem per year (1.0E-04 Sievert per year).

This report was prepared using the format suggested by DOE Headquarters. Section I provides an overview of the INEEL facilities and a brief description of the radioactive materials and processes at the facilities. Section II identifies radioactive air effluent release points (i.e., vents and stacks) and diffuse sources at the INEEL and actual releases during 1997. Section II also describes the effluent control systems for each potential release point. Section III provides the methodology and EDE calculations for 1997 INEEL radioactive emissions. Section IV provides information regarding construction or modification activities that occurred during 1997. Appendix A contains information specific to the Naval Reactors Facility (NRF) located within the INEEL reservation. However, the EDE information for NRF is reported in the EDE for the INEEL in order to demonstrate INEEL compliance with the 40 CFR 61, Subpart H, dose standard of 10 mrem per year (1.0E-04 Sievert per year). Appendix B contains information specific to the INEEL Research Center (IRC) because the IRC is not part of the INEEL's contiguous site (per the NESHAP's definition of "facility"). Appendices C and D provide supporting documentation used as input parameters for the CAP-88 computer code. Data, calculations, model runs, and other documentation used to develop this report are maintained on file at the INEEL in accordance with 40 CFR 61.95 . 


\section{FACILITY INFORMATION}

\section{Site Description}

The Idaho National Engineering and Environmental Laboratory (INEEL) of the Department of Energy (DOE) was established by the federal government in 1949 to conduct research and further the development of nuclear reactors and related equipment. Major DOE Programs at the INEEL include test irradiation services, waste management, light-water-cooled reactor safety testing and research, operation of research reactors, and environmental restoration. The INEEL is operated for DOE by various contractors. Major contractors at the INEEL include Lockheed Martin Idaho Technologies Company (LMICO), Westinghouse Electric Company (WEC), and Argonne National Laboratory (ANL). These contractors conduct the various INEEL programs under the administration of three DOE field offices: Idaho Operations Office (DOE-ID), Chicago Operations Office (DOE-CH), and the Pittsburgh Naval Reactors Idaho Branch Office (PNR). DOE-ID is the primary contact concerning compliance with the National Emission Standards for Hazardous Air Pollutants (NESHAPs) at the INEEL.

The INEEL encompasses approximately 890 square miles on the upper Snake River Plain in southeastern Idaho (Figure 1). The nearest INEEL boundaries to population centers are approximately 22 miles west of Idaho Falls, Idaho, 23 miles northwest of Blackfoot, Idaho, 44 miles northwest of Pocatello, Idaho, 7 miles east of Arco, Idaho, and 1 mile north of Atomic City, Idaho. In addition, individual farms and ranches are located near the INEEL boundaries. These receptors represent the locations used for demonstration of compliance with the NESHAPs' dose standard of 10 mrem per year effective dose equivalent (EDE). Section III provides information concerning distances from the INEEL emission sources to these locations and population densities from each operational area.

Climatology of the INEEL is characterized as semiarid. The losation of the INEEL in a relatively flat valley surrounded by mountains, coupled with its approximately 5,000-foot altitude, affects its overall climate and day-to-day weather systems. Air masses entering the Snake River Plain from the west have lost most of their moisture to precipitation before passing over the INEEL. Therefore, annual precipitation at the INEEL is light. The orientation of the Snake River Plain and bordering mountain ranges channel the winds so that a southwest wind predominates over the INEEL. The second most frequent winds are from the northeast. 


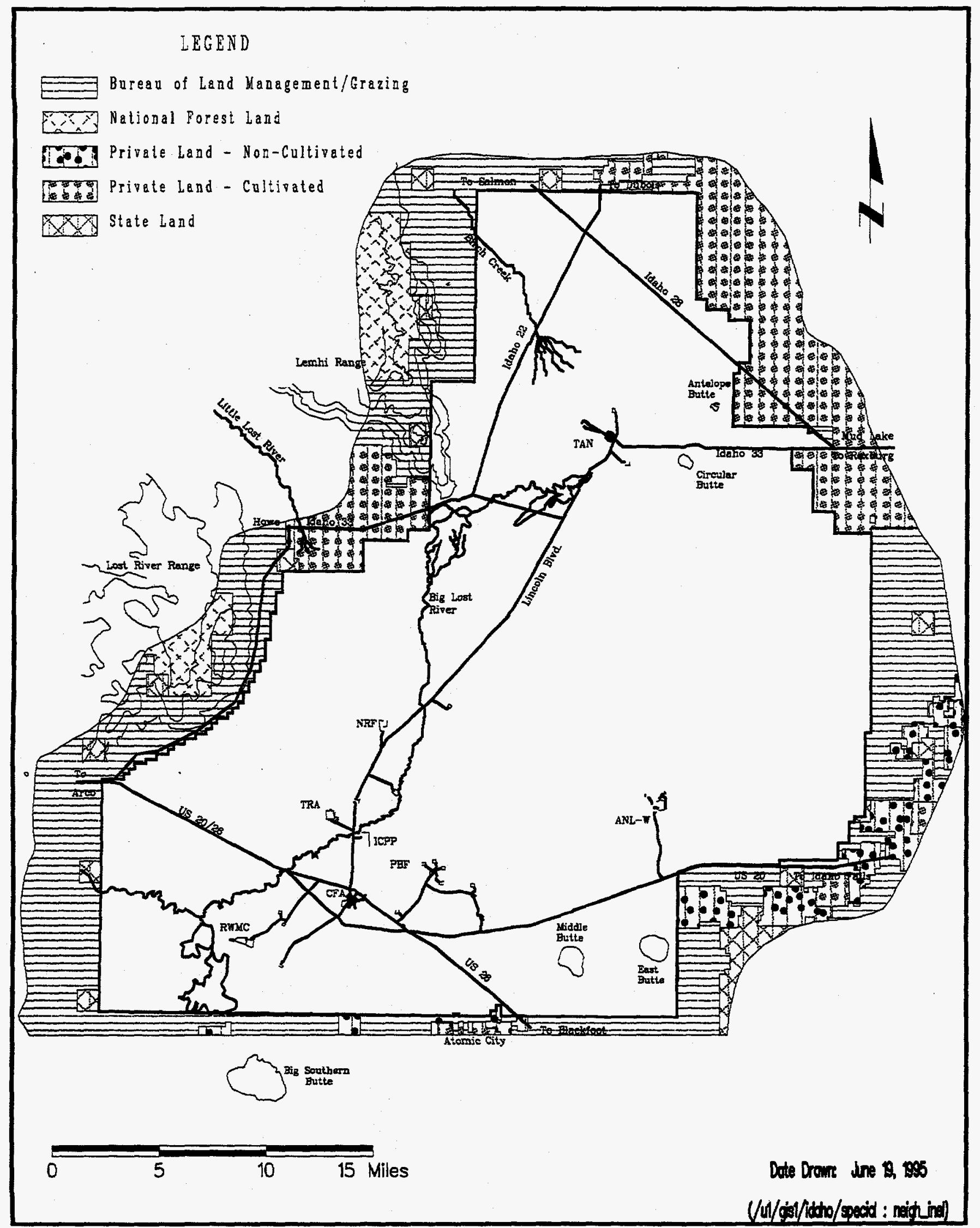

Figure 1. Idaho National Engineering and Environmental Laboratory and Major Facilities. 
The annual average air temperature at the INEEL [measured at the Central Facilities Area (CFA)] is $42.0^{\circ} \mathrm{F}$ $\left(5.6^{\circ} \mathrm{C}\right)$ with extremes of $-47^{\circ} \mathrm{F}\left(-44^{\circ} \mathrm{C}\right)$ and $101^{\circ} \mathrm{F}\left(38^{\circ} \mathrm{C}\right)$. The average annual windspeed at CFA $(20-\mathrm{ft}$ level) is $7.5 \mathrm{mph}$ (3.4 meters per second). The minimum average monthly windspeed of $5.1 \mathrm{mph}$ ( 2.3 meters per second) occurs in December, and the maximum average monthly windspeed of $9.3 \mathrm{mph}$ (4.2 meters per second) occurs in April and May. Calm conditions prevail approximately $11 \%$ of the time (Clawson et al. 1989).

Average annual precipitation over the INEEL (as measured at CFA) is approximately 9 inches. Yearly totals have ranged from 4.5 to 14.4 inches. A portion of this precipitation is received as snowfall, which averages 28 inches per year (Clawson et al. 1989).

Topography of the INEEL is representative of the upper Snake River Plain as a whole. The INEEL surface is a combination of basalt, eolian, and alluvial sedimentary deposits. Vegetation and wildlife on the INEEL are typical of a cool, desert shrub biome.

The INEEL is subject to 40 CFR 61, Subpart H, "National Emission Standards for Emissions of Radionuclides Other Than Radon From Department of Energy Facilities." While the INEEL consists of a variety of operations spread over the 890-square-mile reservation, for purposes of compliance demonstration with the 40 CFR 61, Subpart $H$, dose standard of 10 mrem per year, the INEEL is defined as one facility. The final rule promulgating 40 CFR 61, issued December 15, 1989, specified "... that all buildings, structures and operations within one contiguous site shall be considered a single facility."

Operations that potentially emit radionuclides are located at various areas on the REEL and can be grouped into eight distinct locations:

- Argonne National Laboratory - West (ANL-W)

- Central Facilities Area (CFA)

- Idaho Chemical Processing Plant (ICPP)

- $\quad$ Naval Reactors Facility (NRF).

- Power Burst Facility (PBF) area, including the Waste Reduction Operations Complex (WROC)

- Radioactive Waste Management Complex (RWMC)

- Test Area North (TAN), which encompasses the Specific Manufacturing Capability (SMC) operation

- $\quad$ Test Reactor Area (TRA). 
Figure 1 shows the location of these areas at the INEEL. A more detailed description of each of these areas, excluding the NRF, is provided below. The NRF area description is in Appendix A.

\section{Area and Source Descriptions}

\section{Argonne National Laboratory - West}

The ANL-W site is located on the southeastern corner of the INEEL in Bingham County, Idaho. ANL- W is operated for the DOE by the University of Chicago through the DOE-CH. ANL-W is a research facility that has contributed significantly to knowledge advancements in liquid metal fast breeder reactor technology and extreme condition fuels and reactor materials behavior. The present mission of ANL-W involves research related to spent nuclear fuel and waste treatment technologies, and reactor shutdown activities.

The Experimental Breeder Reactor-II (EBR-II) defueling was completed in calendar year (CY) 1996. The reactor building is still being ventilated and monitored. The continuous monitoring is supplemented with postfiltration sampling and analysis for particulates. CY 1997 ancillary emissions associated with EBR-II operations included limited cover gas pressure releases for maintenance purposes and characterization of potential emissions from a suspect waste tank and fume hood. Characterization of these ancillary emissions for CY 1997 reporting purposes relied on active sampling and conservative industry standard release calculation methodologies.

The Fuel Conditioning Facility (FCF) conducted operations in CY 1997 with EBR-II spent nuclear fuel. The facility is constantly ventilated. The ventilation stream of FCF is filtered and combined with the filtered and previously monitored EBR-II ventilation stream. These combined streams are collectively continuously monitored for all potentially-present radiological emissions. The continuous monitoring is supplemented with sampling and analysis for particulates, radioiodines, and typically gaseous radionuclides. Emissions from FCF during CY 1997 are reported herein.

The Hot Fuel Examination Facility (HFEF), completed and operating since 1975, houses two large shielded hot cells, one each with air and inert atmospheres. Currently, HFEF examination capabilities are being used to characterize mixed wastes within the DOE complex. HFEF emissions are filtered and continuously monitored for all types of potential radiological emissions. The continuous monitoring is supplemented with continuous sampling and subsequent analysis for particulates and radioiodines (both of which typically yield collections below detection limits) and monthly hot cell grab sampling for typically gaseous radionuclides. Particulate emissions from HFEF during CY 1997 are reported herein. Longer-lived gaseous fission product $\mathrm{Kr}-85$ exists in the HFEF inert atmosphere cell and was released in minor amounts as a result of keeping the inert atmosphere cell at a negative pressure. Finally, the small quantity of activation product Ar-41 released from HFEF during CY 1997 is also reported herein. 
The Fuel Manufacturing Facility (FMF) began operation in 1986 as a specialized facility built to cast uranium based fuel for the DOE-complex sodium cooled reactors. Since the shutdown of the EBR-II reactor, FMF has been employed in casting operations in support of waste form development activities as well as Highly Enriched Uranium (HEU) recovery operations. Spent fuel treatment technologies will also be tested at FMF as part of the ongoing ANL-W mission. The FMF is constantly ventilated with its emissions being sampled and analyzed for particulate characterization, the CY 1997 results of which are reported herein.

The Transient Reactor Test Facility (TREAT) reactor did not operate in CY 1997. The building ventilation system was operated only a few hours a month to keep the fan bearings lubricated. The bench-scale Plasma Hearth Process (PHP) was tested during 1997 using nonhazardous test materials but is now shut down. It is not known if the PHP will be restarted. The ventilation system is sampled and analyzed for particulates whenever the fans are operated or when the PHP is in operation. The particulate results reported this year for this stack are from that few hours a month of building ventilation for equipment maintenance purposes.

The Laboratory and Office Building (L\&O) houses the majority of ANL-W's chemical and radiological analytical capabilities. The main hot cell, which vents through the L\&O main stack, was completed in 1964 and is equipped to conduct destructive assays of irradiated fuel and therefore serves as a potential point of release for particulate and longer-lived gaseous fission products. The L\&O main stack is actively monitored and sampled for particulate characterization. A monthly check is also made for $\mathrm{Kr}-85$ release. The nondestructive assay (NDA) wing of the L\&O Building was completed in 1976. Only particulate matter has the potential of being in the NDA ventilation stream, which is filtered and sampled for particulate characterization. The CY 1997 sampling results from both stacks are reported herein.

The Zero Power Physics Reactor (ZPPR) did not operate in CY 1997. Though the reactor is not operating, the building is actively ventilated to minimize the buildup of naturally occurring $\mathrm{Rn}-222$ progeny in the facility, which results from $\mathrm{Rn}-222$ diffusion into the facility from outside soil. The ZPPR facility will also be the site of the electron microscope analyses and other research activities. The ventilation stream is still actively sampled and analyzed for particulate matter, the results of which are reported herein.

The Fuel Assembly and Storage Building (FASB) has been in use since 1972 as a multipurpose facility housing a variety of operations including storage of reactor hardware and blanket subassemblies, assembly of blanket elements and jackets, metallurgical laboratory operations including welding, electron microscope analyses, and special sample preparations involving both irradiated and nonirradiated materials. Spent fuel and waste treatment research are part of the FASB future plans. The FASB is constantly ventilated with its emissions being sampled and analyzed for particulates, the CY 1997 results of which are reported herein.

The Sodium Components Maintenance Shop (SCMS), which began operations in 1977 as a facility for cleansing sodium-laden parts taken from the EBR-II reactor for repair or replacement purposes, continued cleansing 
operations in CY 1997. Emissions from the washing process are sampled and analyzed for particulates with the results reported herein. A series of support tanks containing recycled contaminated ethanol used in the SCMS wash process are not expected to have releases. However, ANL-W very conservatively applies industry standard tank flow through analysis methodologies along with periodic ethanol grab sample analyses to determine worstcase potential emissions from the support tanks.

The Radioactive Liquid Waste Treatment Facility (RLWTF), which began operation in 1982, received and processed 8,381 gallons of aqueous waste from other ANL-W facilities in CY 1997. All emissions were sampled and analyzed for particulates, the results of which are reported herein. All tritium emissions were characterized by liquid batch sampling with the presumption of $100 \%$ release in the form of tritiated water vapor.

\section{Central Facilities Area}

The CFA is located in the south-central section of the INEEL and provides support services for the INEEL. This area includes: (a) DOE Radiological and Environmental Sciences Laboratory (RESL), (b) Environmental Science and Research Foundation (Foundation), (c) maintenance shops, (d) vehicle maintenance facilities, (e) environmental monitoring and calibration laboratories, ( $f$ ) communications and security systems, (g) fire protection, (h) medical services, (i) warehouses, and (j) other support services facilities. With the exception of RESL and the Foundation, CFA is operated for DOE-ID by LMITCO.

Minor releases occur from facilities at CFA where work is routinely conducted with small quantities of radioactive materials. This includes operations at RESL, the Environmental Chemistry Laboratory, and the Health Physics Instrumentation Laboratory (HPIL). RESL provides analytical services for environmental sampling programs. Only small (tracer) quantities of radioactive materials are used at this facility. The HPIL mainly uses sealed sources. Therefore, no routine emissions are associated with these operations.

\section{Idaho Chemical Processing Plant}

The ICPP is located in the south-central portion of the INEEL. The facility was constructed in 1950 and is operated under DOE-ID administration by LMITCO. The ICPP is a multipurpose plant containing approximately 230 buildings and structures.

The mission of the ICPP has changed significantly in light of the decision to discontinue reprocessing of spent nuclear fuel (SNF) in April 1992. Activities having radioactive emissions in 1997 at the ICPP are broken down into the following areas, which are discussed below:

\footnotetext{
- SNF Management

- Waste Management

- Technology Development.
} 
Spent Nuclear Fuel Management. Before 1992, SNF was shipped to the ICPP for temporary storage before reprocessing for uranium recovery. Although fuel reprocessing was discontinued, DOE and Navy fuel continues to arrive for storage. This fuel is transferred to storage until final disposition (e.g., placement in a geologic repository) and conditioning needs and methods have been determined.

Fuel receipt and storage areas include the Fuel Storage Building (CPP-603), the Fluorinel and Storage (FAST) Facility (CPP-666), the Underground Fuel Storage Facility (CPP-749), and the Unirradiated Fuels Storage Facility.(CPP-651). CPP-603 has fuel handling units (FHUs) stored in water pools or in the dry Irradiated Fuel Storage Facility; currently CPP-666 has only wet storage; CPP-749 has only dry storage; and CPP-651 has dry unirradiated fuel storage. All fuel elements are in retrievable storage. No new fuel is being received and transferred into CPP-603 underwater storage basins.

CY 1997 emissions for the FAST facility were based on the FAST Stack monitor. The remaining fuel storage facilities have a constant air monitor (CAM) that is used to determine air emissions, or air emissions are calculated based on process knowledge.

Waste Management. Interim storage space for high-level waste before calcination is provided by eleven 300,000gal underground tanks (WM-180 to -190) and four 18,400-gal tanks (WL-101, WM-100, WM-101, and WM-102) housed in vaults at the north side of the Waste Disposal Building (CPP-604). WM-190 is the designated reserve tank if the other tanks leak. Additional storage can be provided by four 30,000-gal underground tanks (WM-103 to -106$)$ that rest on concrete pads with curbing. These tanks are kept empty and are used only by special DOE authorizations for segregation of wastes from nonroutine campaigns. Emissions from these tanks are monitored via the Main Stack (CPP 708-001).

Calcination activities at the ICPP resumed in 1997. In the calcining process, radioactive liquid mixed with calcium nitrate and sometimes aluminum nitrate is sprayed continuously into a fluidized bed calcining vessel, where the droplets contact hot solid particles. Water evaporates while acids and other compounds decompose. Dissolved solids (i.e., metals and salts) are thus converted to dry, granular, calcined particles. This calcine is stored in stainless-steel bins contained in concrete vaults. Radiological air emissions from the first three bin sets are monitored via the Main Stack (CPP 708-001). Radiological air emissions from the remaining bin sets are negligible and are based on CAM data. Particulate emissions from the calcining off-gas are continuously monitored through the Main Stack per 40 CFR 61 requirements. Emissions from the New Waste Calcining Facility processing cells are continuously compliance monitored on the CPP-659 HVAC stack (CPP-659-33).

Low-level radioactive liquid wastes are collected and concentrated in the High-Level Liquid Waste Evaporator (HLLWE). The concentrate (e.g., bottoms) is then processed as high-level (sodium-bearing) radioactive waste. The overhead products from the HLLWE are processed through the Process Equipment Waste (PEW) Evaporator 
(CPP-604). The concentrate is processed as a high-level radioactive waste. The PEW overheads are processed through the Liquid Effluent Treatment and Disposal (LET\&D) Facility where emissions are accounted for via the ICPP Main Stack.

Minor sources of radioactive emissions from waste management activities include the radiological and hazardous waste accumulation area (CPP-1617), unloading and transfer of low-level radioactive liquids from CPP-1619, the Anti-C/Safety Equipment Handling Facility, and small, miscellaneous emissions from radioactively contaminated buildings and liquids in tanks.

Technology Development. LMITCO technology development efforts support the objectives of safe and efficient interim storage of SNF and radioactive waste, as well as the development of a process or processes to ultimately prepare SNF and radioactive wastes for final disposal.

Most radioactive emissions from these activities are from laboratories and pilot plants in the CPP-602, CPP-627, CPP-637, and CPP-684 buildings. The largest emission among these facilities is from the Remote Analytical Laboratory (RAL), CPP-684.

\section{Power Burst Facility Area}

The PBF area is located in the south-central portion of the INEEL. This area was originally used to support studies of nuclear reactors under normal and off-normal operating conditions. These studies were concluded in 1985 and the PBF area facilities have been modified to accommodate new missions or are no longer in use. The PBF area is operated for the DOE-ID by LMITCO.

The only potential sources of radioactive emissions in the PBF area are the PBF reactor and the Waste Experimental Reduction Facility (WERF). The PBF reactor is currently in a shutdown mode.

The WERF is an experimental facility for research and development of techniques for handling and reducing the volume of radioactive and mixed wastes. Operations at the WERF include metal sizing, compaction, incineration, and stabilization of radioactively contaminated wastes.

Emissions from the PBF area result from WERF operations and periodic maintenance activities at PBF, i.e., cleanup and decontamination activities. Emissions from WERF are extremely small and result from the processing of waste contaminated with fission and activation products. Emissions from the North and East Stacks, which ventilate the incineration and waste handling activities, are continuously monitored. Air emission data for CY 1997 from the PBF area are provided in Section II. 
The RWMC was established in the southwest corner of the INEEL in 1952 to accommodate the radioactive wastes generated by the INEEL operations. In addition to receipt of radioactive wastes generated at the INEEL, the RWMC has also received wastes from other DOE facilities including the Rocky Flats Plant. The RWMC is operated for the DOE-ID by LMTCO.

Areas at the RWMC include the Subsurface Disposal Area (SDA), the Transuranic Storage Area (TSA), the Operations Area and the administrative area. Low-level radioactive wastes are disposed in pits and vaults at the SDA. The TSA includes the Stored Waste Examination Pilot Plant (SWEPP), the Drum Venting Facility (DVF) and the Waste Storage Facility (WSF). Transuranic wastes are stored on an interim basis at the TSA. At the SWEPP facility, transuranic wastes are examined by various nondestructive techniques. A health physics laboratory fume hood is located in the Operations Area. In addition, environmental restoration techniques are being investigated at the RWMC. Emissions from these sources are based on engineering calculations derived from process knowledge with the exception of the DVF, which is based on a CAM.

\section{Test Area North}

The TAN complex is located at the north end of the INEEL. TAN has been used for a variety of projects and currently has two major facilities: SMC facility, and the Technical Support Facility (TSF) TAN Hot Shop. The TAN Hot Shop and the SMC facility are both operated for DOE-ID by LMITCO.

The TSF TAN Hot Shop contains decontamination and hot cell areas. Operations include decontamination of radioactively contaminated equipment and remote examination of radioactive materials.

The SMC Project is a multiphased manufacturing operation that produces an armor package for the U.S. Department of the Army. The SMC Project was assigned to the INEEL in mid-1983. Several existing facilities were modified and new facilities constructed to contain state-of-the-art manufacturing and processing equipment. The SMC Project consists of the Materials Development Facility (MDF), TAN-629 Fabrication and Assembly, TAN-679 Rolling Operation, and support facilities.

The MDF was established to support the identification, evaluation, and development of manufacturing processes for the SMC Project. Operations involve fabrication and assembly work to produce test-size armor assemblies. Standard metal working equipment such as punches, shears, brakes, and lasers are used to fabricate DU material. Other activities include development of tools and fixtures, and preparation and testing of metallurgical specimens. 
The TAN-629 facility, located within the TAN hangar, contains production unit areas and space for offices, support functions, and service areas. TAN-675 is located on the north side of the hangar and houses utilities. TAN-677 is located on the south side for truck receiving and controlled access to the facility.

Production units are semiautomatic systems that perform the same types of operations as MDF, except on an automated basis to produce full-size assemblies at a higher throughput. The depleted uranium (DU) parts are sheared, punched, laser cut, cleaned, and painted. The assemblies are packaged and shipped to their final destination.

The TAN-679 facility consists of a manufacturing area and a Process Reclamation Facility (PRF), TAN-681. TAN-679 operations include a process production line, office area, support functions, and service areas. Depleted uranium (DU) material is processed and subsequently used as feedstock for TAN-629 operations. Processes include a preheat and hot roll operation, shearing to length and width, acid etching, water rinsing, and final inspection.

The PRF operations include the collection, recycling, and disposition of waste material. All contaminated liquid waste streams are collected in storage tanks for treatment/reuse.

Radiological air emissions from the TAN area are associated with the Hot Shop operations and the SMC. Potential emissions from the Hot Shop area include noble gases, iodines, and particulates. Emissions from this facility are operation specific and are not associated with any continuous process. Section II provides data on air emissions from the TAN Hot Shop area for CY 1997.

Radiological air emissions from SMC are associated with processing of DU. Potential emissions are uranium isotopes and associated radioactive progeny. Section II provides data on air emissions from the SMC for CY 1997.

\section{Test Reactor Area}

The TRA is located in the south-central section of the INEEL near the ICPP. Operations at TRA are conducted by LMITCO, under the administration of DOE-ID. One activity, the TRA Hot Cells, are operated by International Isotopes Idaho, Inc. under contract to LMITCO. The TRA has facilities for studying the performance of reactor materials and equipment components under high neutron flux conditions. The major facility at TRA is the Advanced Test Reactor (ATR). Other operations at TRA include hot cell operations, research and development, site remediation, and analytical laboratory services.

Radioactive emissions from the TRA are primarily associated with operation of the ATR. These emissions include noble gases, iodines, and other mixed fission and activation products. Other radioactive emissions are 
associated with hot cell operations, sample analysis, site remediation, and research and development activities. Air emission data for TRA are provided in Section II. 


\section{AIR EMISSIONS DATA}

As discussed in Section I, the INEEL contains a number of operations that have the potential to emit radionuclides. Tables II-1 through II-7 identify all stacks and vents at the INEEL that represent potential radiological air emission sources. Data on NRF emission sources are provided in Appendix A. The tables are organized according to the major INEEL operational areas described in Section I. The tables describe each potential emission point and the effluent controls and their efficiencies, if applicable. The tables also identify emission sources that require monitoring and/or sampling on a continuous basis.

The nearest receptor to the operational area is listed at the top of each table. Information on the INEEL maximally exposed individual (MEI), as well as location from these operational areas to the INEEL MEI, can be found in Section III. Not all potential radionuclide emission points are monitored on a continuous basis at INEEL. Section 61.93(b) of 40 CFR 61, Subpart $H$, provides prescriptive requirements for continuous monitoring of those emission points that have a potential to emit radionuclides in quantities that could result in an EDE to a member of the public in excess of $1 \%$ of the NESHAPs' dose standard of 10 mrem (1.0E-04 Sievert) per year, i.e., 0.1 mrem (1.0E-06 Sievert) per year. In evaluating the potential of a release point to discharge radionuclides into the air for the purposes of this regulation, the estimated radionuclide release rates are based on the discharge of the effluent stream that would result if all pollution control equipment did not exist but the facility operations were otherwise normal [40 CFR 61.93(b)(4)(ii)]. All other potential emission sources require periodic confirmatory measurements to verify the low emissions.

In response to NESHAPs' requirements, the INEEL conducted a study to determine radiological emission points subject to the 40 CFR 61.93(b) monitoring requirements. The methodology and results of the evaluation are presented in the report DOE/ID-10310, NESHAPs 40 CFR 61.93 Monitoring Requirements for Radiological Emission Sources at INEEL, November 1990. A reassessment of all the INEEL emission sources was completed in 1992 at the request of Environmental Protection Agency (EPA) Region X. In addition, the INEEL has implemented a "periodic confirmatory measurements" program in response to these 40 CFR 61.93(b) requirements.

The above evaluations identified six emission points that require continuous monitoring under 40 CFR 61.93(b). The six sources are: ICPP Main Stack (CPP-708-001), New Waste Calcining Facility HVAC Stack (CPP-659033) [Note: This source was redesignated as requiring continuous monitoring under 40 CFR 61.93(b) in 1997], WERF north stack (PER-755-001), WERF east stack (PER-765-001), HFEF main stack (ANL-785-018), and ANL-W 200-ft stack (ANL-764-001). All other potential emission points were determined to have potential EDEs of less than 0.1 mrem (1.0E-06 Sievert) per year (Actual emissions from these points are based on sampling and/or engineering calculations based on process knowledge). 
Tables II-8 and II-9 list the point source radionuclide emissions for CY 1997 associated with each operational area at the INEEL. Table II-8 lists emissions from all continuous compliance monitored sources, and Table II-9 lists the estimated radionuclide emissions from all other point sources. Table II-10 lists the nonpoint source radionuclide emissions for CY 1997 at the INEEL.

In some cases, results shown in Table II- 8 and Table II-9 are reported as gross alpha, gross beta, and gross beta/gamma. In these instances, these emissions were assumed to be plutonium-239 for gross alpha and strontium-90 in equilibrium with yttrium-90 for gross beta and gross beta/gamma. This conservative assumption was based on the fact that these two radionuclides have extremely low concentration values in Table 2 of 40 CFR 61, Appendix E. However, it is not expected that these isotopes are always present due to the nature of the varying operations at these INEEL facilities. 
Table II-1. ANL-W Radiological Air Emission Sources That Had Emissions In CY 1997

\begin{tabular}{|c|c|c|c|c|c|c|}
\hline \multicolumn{7}{|c|}{ 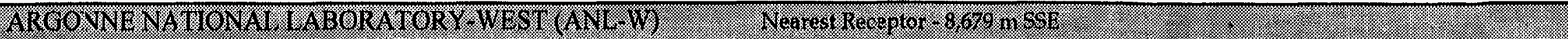 } \\
\hline 4ivis. & 6 & 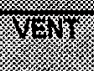 & 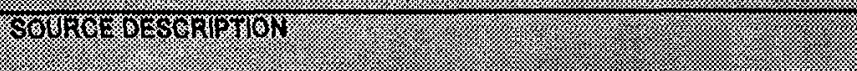 & 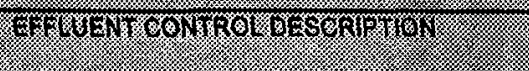 & 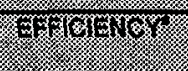 & 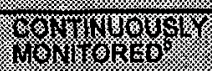 \\
\hline ANL & 704 & 008 & FUEL MANUFACTURING FACILITY (FMF) MAIN STACK & $\begin{array}{l}\text { CASTING AREA - TWO HEPA FILTER } \\
\text { BANKS IN SERIES } \\
\text { GLOVE BOX AREA - TWO HEPA FILTER } \\
\text { BANKS IN SERIES } \\
\text { REMAINDER - HEPA FILTER BANK }\end{array}$ & $.99 .97 \% \mathrm{EACH}$ & \\
\hline$\overline{\text { ANL }}$ & 752 & 004 & MAIN LABORATORY AND OFFICE (L\&O) BUILDING STACK & TWO HEPA FILTER BANKS IN SERIES & $99.97 \%$ EACH & \\
\hline$\overline{\text { ANL }}$ & 752 & 005 & $\begin{array}{l}\text { NONDESTRUCTIVE ASSAY LABORATORY STACK IN THE LABORATORY } \\
\text { AND OFFICE (L\&O) BUILDING }\end{array}$ & $\begin{array}{l}\text { EFL AREA - TWO HEPA FILTER BANKS IN } \\
\text { SERIES } \\
\text { REMAINDER - HEPA FILTER BANK }\end{array}$ & $99.97 \%$ EACH & \\
\hline$\overline{\text { ANL }}$ & 764 & 001 & $\begin{array}{l}\text { FCF AND EBR-II REACTOR EMISSIONS EXHAUST THROUGH THIS 200- } \\
\text { FOOT MAIN STACK }\end{array}$ & $\begin{array}{l}\text { EBR- II- HEPA FILTER BANK } \\
\text { FCF HOT CELL - TWO HEPA FILTER BANKS } \\
\text { IN SERIES } \\
\text { REMAINDER OF FCF - HEPA FILTER BANK }\end{array}$ & 99.97\% EACH & $\bar{x}$ \\
\hline$\overline{\text { ANL }}$ & 766 & 056 & SECONDARY ARGON COVER GAS PURGE VENT & NONE & NONE & \\
\hline$\overline{\text { ANL }}$ & 768 & 105 & $\begin{array}{l}\text { SUSPECT WASTE TANK FROMDECONTAMINATION SHOWER IN } \\
\text { HEALTH PHYSICS AREA OF POWER PLANT }\end{array}$ & HEPA FILTER BANK & $99.97 \%$ & \\
\hline$\overline{\text { ANL }}$ & 768 & 108 & HPAREA FUMEHOOD IN POWER PLANT & HEPA FILTER BANK & $99.97 \%$ & \\
\hline$\overline{\text { ANL. }}$ & 777 & 002 & ZPPR & $\begin{array}{l}\text { REACTOR CELL - TWO HEPA FILTER } \\
\text { BANKS IN SERIES } \\
\text { REMAINDER - HEPA FILTER BANK }\end{array}$ & $99.97 \%$ EACH & \\
\hline$\overline{\text { ANL }}$ & 785 & 018 & HOT FUEL EXAMINATION FACILITY (HFEF) MAIN STACK ${ }^{c}$ & $\begin{array}{l}\text { HOT CELLS - TWO HEPA FILTER BANKS IN } \\
\text { SERIES } \\
\text { REMAINDER - HEPA FILTER BANK }\end{array}$ & $99.97 \%$ EACH & $\bar{x}$ \\
\hline$\overline{\text { ANL }}$ & 787 & 001 & FUEL ASSEMBLY AND STORAGE BUILDING (FASB) MAIN STACK & HEPA FILTER BANK & $99.97 \%$ & 7 \\
\hline$\overline{\text { ANL }}$ & 793 & 001 & SODIUM COMPONENTS MAINTENANCE SHOP (SCMS) MAIN STACK & HEPA FILTER BANK & $99.97 \%$ & \\
\hline$\overline{\text { ANL }}$ & $793 A$ & 027 & ALCOHOL STORAGE TANK VENTS & NONE & NONE & \\
\hline$\overline{\text { ANL }}$ & 798 & 017 & RADIOACTIVE LIQUIO WASTE EVAPORATOR EXHAUST & $\begin{array}{l}\text { EVAPORATORS - TWO HEPA FILTER } \\
\text { BANKS IN SERIES } \\
\text { REMAINDER - HEPA FILTER BANK }\end{array}$ & $99.97 \% \mathrm{EACH}$ & \\
\hline \multicolumn{7}{|c|}{ 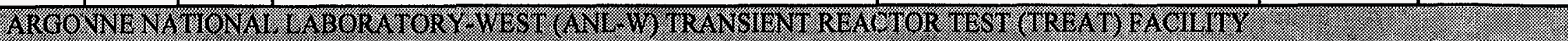 } \\
\hline ANL & 720 & 007 & $\begin{array}{l}\text { TRANSIENT REACTOR TEST FACILITY REACTOR COOLING AIR } \\
\text { EXHAUST }\end{array}$ & TWO BANKS HEPA FILTERS IN SERIES & $99.97 \%$ EACH & \\
\hline
\end{tabular}


Table Il-1. ANL-W Radiological Air Emission Sources which had Emissions in CY 1997 (continued)

a. A single HEPA filter will remove $99.97 \%$ of particulates that are 0.3 microns in diameter or greater.

b. Alr emission sources that require continuous monitoring are based on an unabated emission potential of 0.1 mrem/yr or greater.

b. Air emlssion sources that require continuous monitoring are based on an unabated emission potential of $0.1 \mathrm{mrem} / \mathrm{yr}$ or greater.

(1) Building-Laboratory exhaust system passes through one bank of dioctyl phthalate (DOP)-tested HEPA filters; $42,875 \mathrm{cfm}$

(2) Main Cell exhaust nows through three banks of HEPA nilers, only one bank of Which is DOP-tested; $0-500 \mathrm{cfm}$

d. Three Decon Cell exhaust flows through three stages of HEPA filers, two stages of which are DOP-tested; $3030-3860 \mathrm{cfm}$.

(1) Water Wash vessel exhaust flows through one slage of DOP-lested HEPA filters.

(2) High Bay ventilation is also filtered by a single DOP-tested HEPA filter bank.

(3) Process ventilation is double (DOP-lested) HEPA filtered. 
Table II-2. CFA Radiological Air Emission Sources

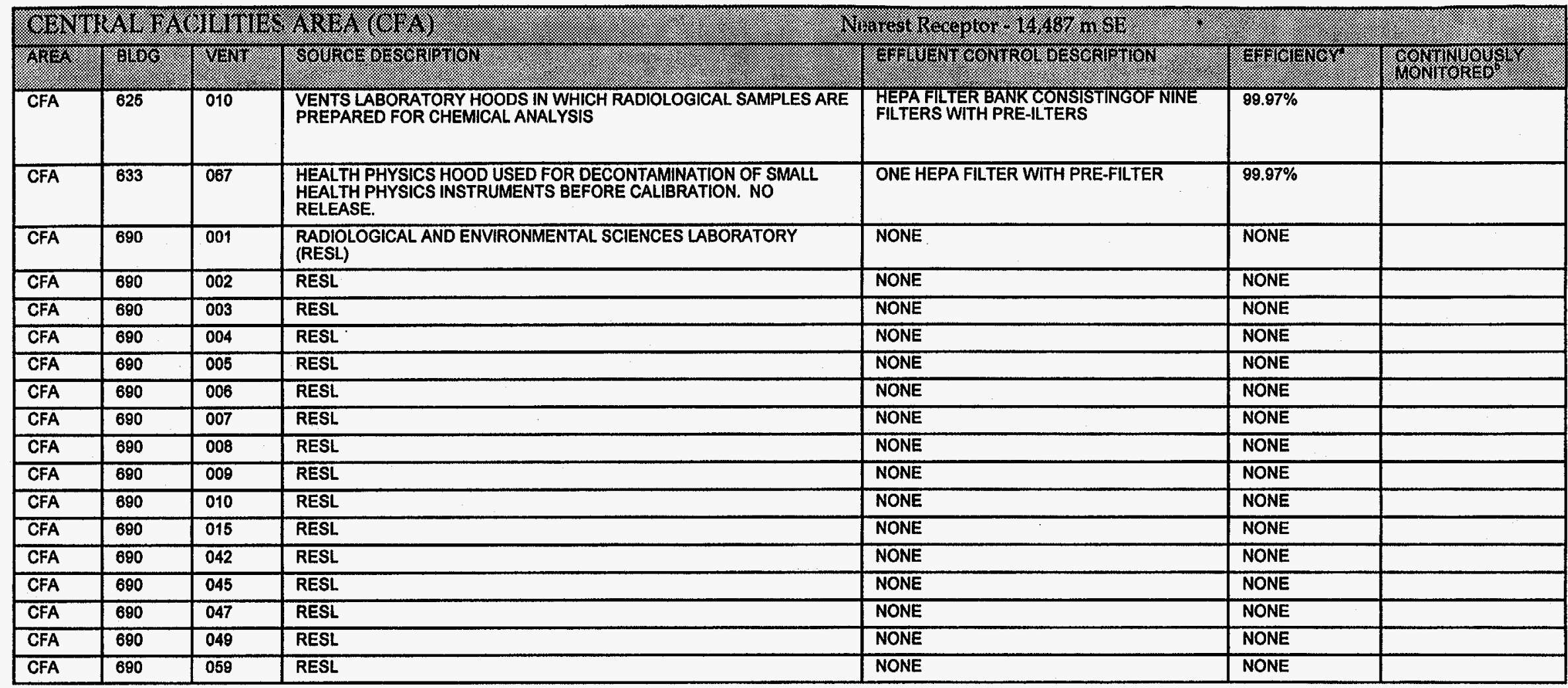

a. A single HEPA filter will remove $99.97 \%$ of particulates that are 0.3 microns in diameter.

b. Air emission sources that require continuous monitoring are based on an unabated emission potential of $0.1 \mathrm{mrem} / \mathrm{yr}$ or greater. 
Table II-3. ICPP Radiological Air Emission Sources

\begin{tabular}{|c|c|c|c|c|c|c|}
\hline \multicolumn{7}{|c|}{ 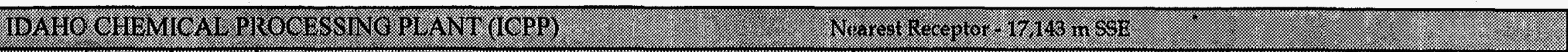 } \\
\hline$\sqrt{18}=8$. & 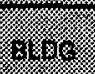 & 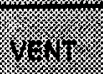 & 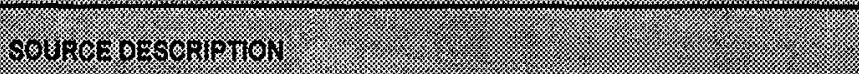 & 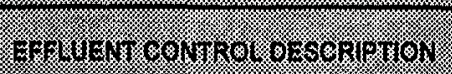 & etrocterier & 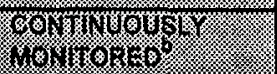 \\
\hline CPP & 601 & 024 & HEXONE STORAGE AND FEED TANKS & NONE & NONE & \\
\hline CPP & $\overline{602}$ & $\overline{012}$ & $\begin{array}{l}\text { MAIN LAB EXHAUST FROM LABORATORY HOODS, GLOVE BOXES AND } \\
\text { DENITRATOR IN BUILDING } 602\end{array}$ & $\begin{array}{l}\text { HEPA FILTER OR } \\
\text { TWO HEPA FILTERS IN SERIES }\end{array}$ & $\begin{array}{l}99.97 \% \\
\text { each filter }\end{array}$ & \\
\hline CPP & $\overline{602}$ & 014 & LABORATORY 224 CAVE IN 602 & HEPA FILTER & $89.97 \%$ & \\
\hline$\overline{C P P}$ & 602 & $\overline{031}$ & PERCHLORIC ACID HOOD EXHAUST IN 602 & DEMISTER & $\overline{\text { NONE }}$ & \\
\hline CPP & $\overline{603}$ & 001 & IFSF & HEPA FILTER & $99.97 \%$ & \\
\hline$\overline{C P P}$ & $\overline{603}$ & $\overline{019}$ & UNDERWATER FUEL STORAGE AREA & NONE & NONE & \\
\hline CPP & 827 & 007 & $\begin{array}{l}\text { VENTS LABORATORY HOODS AND RADIOACTIVE GLOVE BOXES } \\
\text { (SPECIAL ANALYSIS LAB, ACID FUME HOODS) }\end{array}$ & $\begin{array}{l}\text { HEPA FILTER OR } \\
\text { TWO HEPA FILTERS IN SERIES }\end{array}$ & $\begin{array}{l}99.97 \% \\
\text { each filter }\end{array}$ & \\
\hline CPP & 627 & 008 & $\begin{array}{l}\text { SPECIALANALYSIS LABORATORY HOODS, EMISSIONS } \\
\text { SPECTROSCOPY CAVE }\end{array}$ & TWO HEPA FILTERS IN SERIES & $\begin{array}{l}89.97 \% \\
\text { each filter }\end{array}$ & \\
\hline CPP & 627 & 010 & MULTICURIE CELL & HEPA FILTER & $99.97 \%$ & \\
\hline CPP & 627 & 013 & $\begin{array}{l}\text { HOT CHEMISTRY LAB HOODS, GLOVE BOXES - DECON DEVELOPMENT } \\
\text { LAB }\end{array}$ & HEPA FILTER & $99.97 \%$ & \\
\hline CPP & 627 & 016 & $\begin{array}{l}\text { LABORATORY AIR SAMPLING SYSTEM USED FOR WET CHEMISTRY OF } \\
\text { RADIOACTIVE SAMPLES - FASS }\end{array}$ & HEPA FILTER & $99.97 \%$ & \\
\hline CPP & 630 & 011 & LABORATORY HOODS AND OTHER EXHAUSTS FROM LABS IN 630 & TWO HEPA FILTERS IN SERIES & $\begin{array}{l}89.97 \% \\
\text { each filter }\end{array}$ & \\
\hline CPP & 830 & $\overline{012}$ & $\begin{array}{l}\text { LABORATORY HOODS AND EXHAUSTS FROM PART OF BUILDING } 602 \text { - } \\
300 \text { LABORATORIES }\end{array}$ & TWO HEPA FILTERS IN SERIES & $\begin{array}{l}99.97 \% \\
\text { each filter }\end{array}$ & \\
\hline CPP & 637 & 010 & $\begin{array}{l}\text { MAIN EXHAUST FOR ALL FUME HOODS IN LABORATORY SECTION OF } \\
\text { BUILDING (7 LABS, } 22 \text { HOODS) }\end{array}$ & $\begin{array}{l}\text { HEPA FILTER OR } \\
\text { TWO HEPA FILTERS IN SERIES }\end{array}$ & $\begin{array}{l}99.97 \% \\
\text { each filter }\end{array}$ & \\
\hline CPP & 848 & 002 & VENT FOR THE SLUDGE STORAGE TANK (VES-SFE-106) & $\begin{array}{l}\text { HEPA FILTER } \\
\text { (NOT TESTED) }\end{array}$ & $99.97 \%$ & \\
\hline CPP & 659 & $\overline{033}$ & $\begin{array}{l}\text { EXHAUUSTS BUILDING VENTILATION AIR FROM THE CALCINER AREA. } \\
\text { DEBRIS TREATMENT FACILITY CONSISTING OF DECONTAMINATION } \\
\text { OPERATIONS. }\end{array}$ & TWO HEPA FILTERS IN SERIES & $\begin{array}{l}99.97 \% \\
\text { each filter }\end{array}$ & $\bar{x}$ \\
\hline CPP & 859 & 038 & $\begin{array}{l}\text { EXHAUSTS BUILDING VENTILATION AIR FROM THE DECONTAMINATION } \\
\text { AREA }\end{array}$ & TWO HEPA FILTERS IN SERIES & $\begin{array}{l}89.97 \% \\
\text { each filter }\end{array}$ & \\
\hline CPP & 683 & 002 & 683 HOT SHOP EXHAUST & HEPA FILTER & $99.97 \%$ & \\
\hline$\overline{\mathrm{CPP}}$ & 684 & 001 & REMOTE ANALYTICAL LABORATORY (RAL) & TWO HEPA FILTERS IN SERIES & $\begin{array}{l}99.97 \% \\
\text { each filter }\end{array}$ & \\
\hline
\end{tabular}


Table II-3. ICPP Radiological Air Emission Sources (continued)

\begin{tabular}{|c|c|c|c|c|c|c|}
\hline ISY & S. WIII & (3). & 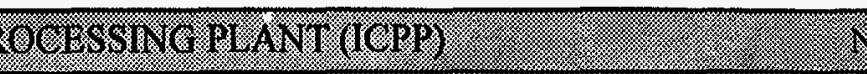 & 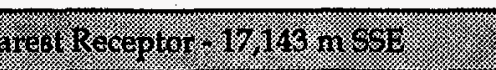 & & \\
\hline Arex & 8006 & $\sqrt{12}+1)$ & 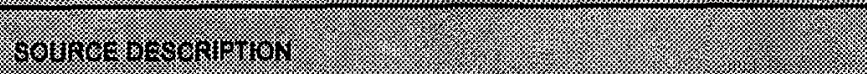 & 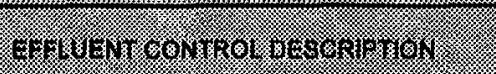 & Eticisicer: & 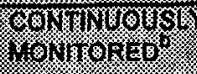 \\
\hline CPP & 694 & 007 & TANK VENTS FOR ORGANIC SOLVENT STORAGE TANK ${ }^{\circ}$ & TWO HEPA FILTERS IN SERIES & $\begin{array}{l}99.97 \% \\
\text { each filter }\end{array}$ & \\
\hline CPP & 694 & 008 & TANK VENTS FOR ORGANIC SOLVENT STORAGE TANK ${ }^{\circ}$ & TWO HEPA FILTERS IN SERIES & $\begin{array}{l}89.97 \% \\
\text { each filter }\end{array}$ & \\
\hline CPP & 684 & 009 & $\begin{array}{l}\text { EXHAUST VENTILATION AIR FROM THE ORGANIC SOLVENT STORAGE } \\
\text { AANK BUILDING }\end{array}$ & $\begin{array}{l}\text { HEPA FILTER } \\
\text { (NOT TESTED) }\end{array}$ & $89.97 \%$ & \\
\hline CPP & 684 & 010 & TANK VENTS FOR ORGANIC SOLVENT STORAGE TANK & TWO HEPA FILTERS IN SERIES & $\begin{array}{l}99.97 \% \\
\text { each filter }\end{array}$ & \\
\hline CPP & 708 & 001 & CPP MAIN STACK INCLUDING THE NEW WASTE CALCINING FACILITY & $\begin{array}{l}\text { HEPA FILTER OR UP TO } \\
\text { THREE HEPA FILTERS IN SERIES }\end{array}$ & $\begin{array}{l}89.97 \% \\
\text { each filter }\end{array}$ & $\bar{x}$ \\
\hline$\overline{\mathrm{CPP}}$ & 732 & 001 & $\begin{array}{l}\text { BIN SET \#1 - VENTS THE VAULT SURROUNDING THE STORAGE BINS } \\
\text { (NOT FLTERED). VAULT AREA NO LONGER A SOURCE. STORAGE BINS } \\
\text { SINGLE HEPA.FILTERED IN POG APS. }\end{array}$ & $\begin{array}{l}\text { NONE (VAULT) } \\
\text { HEPA FILTER (BINS) }\end{array}$ & $99.97 \%$ & \\
\hline CPP & $\overline{742}$ & 001 & $\begin{array}{l}\text { BIN SET \#2 - VENTS THE VAULT SURROUNDING THE STORAGE BINS } \\
\text { (NOT FILTERED). VAULT AREA NO LONGER A SOURCE. STORAGE BINS } \\
\text { SINGLE HEPA-FILTERED IN POG APS. }\end{array}$ & $\begin{array}{l}\text { NONE NAULT) } \\
\text { HEPA FILTER (BINS) }\end{array}$ & $99.97 \%$ & \\
\hline CPP & 746 & 001 & $\begin{array}{l}\text { BIN SET \#3 - VENTS THE VAULT SURROUNDING THE STORAGE BINS } \\
\text { (NOT FILTERED) VAULT AREA NO LONGER A SOURCE. STORAGE BINS } \\
\text { SINGLE HEPA-FLTTERED IN POG APS. }\end{array}$ & $\begin{array}{l}\text { NONE (VAULT) } \\
\text { HEPA FILTER (BINS) }\end{array}$ & $99.97 \%$ & \\
\hline CPP & 749 & 001 & SPENT FUEL STORAGE VAULKTS & $\begin{array}{l}\text { HEPA FILTER } \\
\text { ((NOT TESTED) }\end{array}$ & $99.97 \%$ & \\
\hline CPP & 760 & 002 & $\begin{array}{l}\text { BIN SET \#4 - VENTS THE VAULT SURROUNDING THE STORAGE BINS } \\
\text { (NOT HEPA-FLTTERED) AND THE STORAGE BINS (TWO HEPA FLTTERS } \\
\text { INSTALLED BUT NOT TESTED) }\end{array}$ & $\begin{array}{l}\text { NONE (VAULT) } \\
\text { TWO HEPA FILTERS IN SERIES } \\
\text { INSTALLED BUT NOT TESTED }\end{array}$ & NONE & : \\
\hline CPP & 764 & 002 & VENT FOR VAULT CONTAINING HOT WASTE TANK (NES-SFE-126) & $\begin{array}{l}\text { HEPA FILTER } \\
\text { (NOT TESTED) }\end{array}$ & $89.97 \%$ & \\
\hline$\overline{C P P}$ & 767 & 001 & $\begin{array}{l}\text { FAST STACK. VENTS THE FLUORINEEL AND STORAGE (FAST) } \\
\text { FACILITY. }\end{array}$ & $\begin{array}{l}\text { HEPA FILTER OR TWO HEPA FILTERS IN } \\
\text { SERIES }\end{array}$ & $\begin{array}{l}99.97 \% \\
\text { each filter }\end{array}$ & \\
\hline
\end{tabular}


Table II-3. ICPP Radiological Air Emission Sources (continued)

\begin{tabular}{|c|c|c|c|c|c|c|}
\hline \multicolumn{7}{|c|}{ 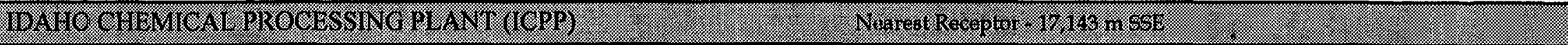 } \\
\hline 4hesing & 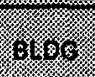 & 2. & 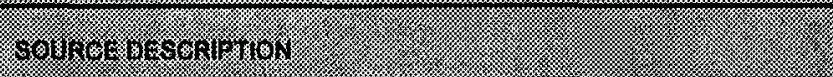 & 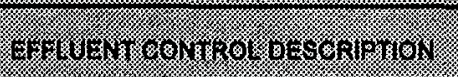 & 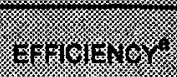 & 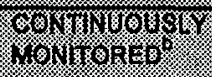 \\
\hline CPP & 791 & 004 & $\begin{array}{l}\text { BIN SET \#6 - VENTS THE VAULT SURROUNDING THE STORAGE BINS } \\
\text { AND THE STORAGE BINS }\end{array}$ & $\begin{array}{l}\text { NONE (VAULT) } \\
\text { TWO HEPA FILTERS IN SERIES (BINS) }\end{array}$ & $\begin{array}{l}99.97 \% \\
\text { each filter }\end{array}$ & \\
\hline CPP & 795 & 004 & BIN SET \#7 - INACTIVE & INACTIVE & & \\
\hline CPP & 1608 & 001 & MANIPULATOR REPAIR CELL & TWO HEPA FILTERS IN SERIES & $\begin{array}{l}98.97 \% \\
\text { each filter }\end{array}$ & \\
\hline CPP & 1611 & $\begin{array}{l}\text { AREA } \\
1\end{array}$ & PERCOLATION POND 2 (NONPOINT SOURCE) & NONE & NONE & \\
\hline$\overline{\mathrm{CPP}}$ & 1612 & $\begin{array}{l}\text { AREA } \\
1\end{array}$ & PERCOLATION POND 1 (NONPOINT SOURCE) & NONE & NONE & \\
\hline CPP & 1817 & 001 & RADIOLOGICAL AND HAZARDOUS WASTE ACCUMULATION AREA & NONE & NONE & \\
\hline CPP & 1818 & 001 & UNLOADING AND TRANSFER OF LOW-LEVEL RADIOACTIVE LIQUIDS & HEPA FILTER & $\begin{array}{l}99.97 \% \\
\text { each filter }\end{array}$ & \\
\hline CPP & 1646 & 001 & ANTI-C/SAFETY EQUIPMENT HANDLING FACILITY & TWO HEPA FILTERS IN SERIES & $\begin{array}{l}89.97 \% \\
\text { each filter }\end{array}$ & \\
\hline
\end{tabular}

a. A single HEPA filter will remove $99.97 \%$ of particulates that are $0.3 \mathrm{~m}$ mcrons in dlameter.

b. Air emission sources that require continuous monitoring are based on an unabated emlssion potential of $0.1 \mathrm{mrem} / \mathrm{yr}$ or greater.

c. Not a source term for 1997 . 
Table Il-4. PBF Area Radiological Air Emission Sources

\begin{tabular}{|c|c|c|c|c|c|c|}
\hline \multicolumn{7}{|c|}{ POW WR BURST FACIITY (PBI) AREA } \\
\hline Riter & 8069 & Tin: & 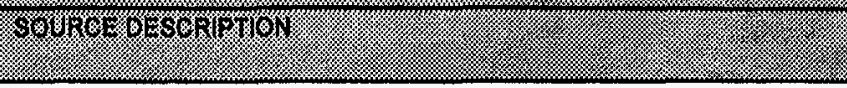 & 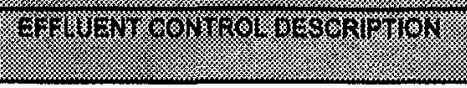 & 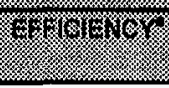 & 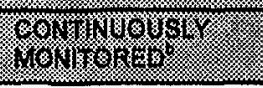 \\
\hline PER & 613 & 021 & $\begin{array}{l}\text { REPACKAGING BOOTH IUSED TO REPACKAGE MIXED WASTE, } \\
\text { CHARACTERIZE MIXED WASTE AND VERIFY WASTE CONTENTS }\end{array}$ & ONE BANK HEPA FILTERS & $99.97 \%$ & \\
\hline PER & 620 & 016 & PBF REACTOR MAIN STACK - REACTOR IS ON STANDBY & $\begin{array}{l}\text { ONE BANK HEPA FLLTERS } \\
\text { ONE BANK SILVER ZEOLITE FILTER }\end{array}$ & $\begin{array}{l}99.97 \% \\
\text { each filter } \\
98.5 \%\end{array}$ & \\
\hline PER & $765^{\circ}$ & $001^{\circ}$ & $\begin{array}{l}\text { VENTS COMPACTOR AND NEW SIZING ROOM. WERF EXHAUST EAST } \\
\text { STACK. }\end{array}$ & $\begin{array}{l}\text { TWO BAG HOUSE FILTERS IN SERIES } \\
\text { ONE HEPA FILTER BANK. }\end{array}$ & $\begin{array}{l}90 \% \\
99.87 \%\end{array}$ & $\bar{x}$ \\
\hline PER & 755 & 001 & WERF EXHAUST NCRTH STACK & $\begin{array}{l}\text { BAG HOUSE FLLTER } \\
\text { ONE HEPA FILTER BANK }\end{array}$ & $\begin{array}{l}90 \% \\
99.87 \%\end{array}$ & $\mathbf{x}$ \\
\hline 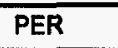 & 758 & 001 & WERF EXHAUST SOUTH STACK & ONE BANK HEPA FILTERS & $99.97 \%$ & \\
\hline
\end{tabular}

a. A single HEPA filter will remove $99.97 \%$ of particulates that are 0.3 microns in dlameter.

b. Alr emission sources that require continuous monitoring are based on an unabated emlssion potential of $0.1 \mathrm{mrem} / \mathrm{yr}$ or greater.

c. This emission source replaces PER-622-003. 
Table II-5. RWMC Radiological Air Emission Sources

\begin{tabular}{|c|c|c|c|c|c|c|}
\hline \multicolumn{7}{|c|}{ 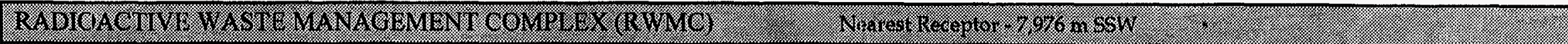 } \\
\hline (18) & 0400 & 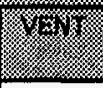 & 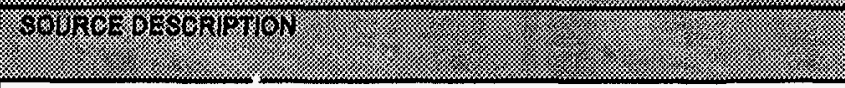 & (4F & 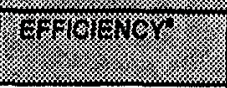 & 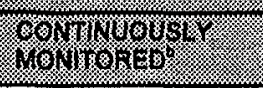 \\
\hline WMF & 601 & 000 & $\begin{array}{l}\text { HP LABORATORY HOOD USED FOR ENVIRONMENTAL SAMPLE } \\
\text { PREPARATION FOR RADIOLOGICAL ANALYSIS }\end{array}$ & ONE $24 \times$ 24-INCH HEPA FILTER & $99.97 \%$ & \\
\hline WMF & 815 & 001 & $\begin{array}{l}\text { DRUM VENTING FACILITY STACK \& HEAD SPACE GAS SAMPLING AND } \\
\text { CONTROL SYSTEM }\end{array}$ & $\begin{array}{l}\text { ONE INLINE HEPA FILTER TO STACK } \\
\text { ONE HEPA FILTER IN SERIES WITH A BANK } \\
\text { (THREE) OF ADSORBERS }\end{array}$ & $\begin{array}{l}89.97 \% \\
\text { each filter }\end{array}$ & \\
\hline WMF & & & WASTE CHARACTERIZATION AND SEGREGATION TENT & HEPA FILTERED & $89.97 \%$ & \\
\hline WMF & & & $\begin{array}{l}\text { OU 7-08 THREE VAPOR VACUUM EXTRACTION TREATMENT UNITS } \\
\text { (MOBILE CERCLA UNITS) }\end{array}$ & NONE & NONE & \\
\hline
\end{tabular}

a. A single HEPA filter will remove $89.97 \%$ of particulates that are 0.3 microns in diameter.

b. Air emission sources that require continuous monitoring are based on an unabated emlssion polential of $0.1 \mathrm{mrem} / y \mathrm{r}$ or greater. 


\section{Table II-6. TAN Radiological Air Emission Sources}

\begin{tabular}{|c|c|c|c|c|c|c|}
\hline \multicolumn{7}{|c|}{ 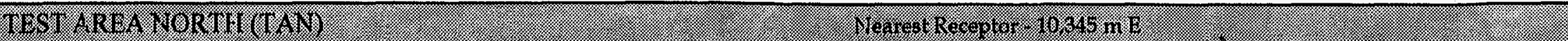 } \\
\hline 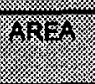 & G19.8 & VI: & G & 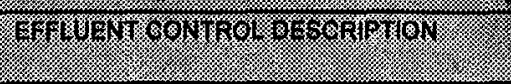 & 40 & 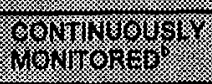 \\
\hline TAN & 607 & 049 & $\begin{array}{l}\text { HOT SHOP CHANGE ROOM EXHAUST - ROOM EXHAUST FOR STEP-OFF } \\
\text { PAD AREA FOR HOT SHOP. INSIDE OF VENT IS CONTAMINATED. THERE } \\
\text { IS A POTENTIAL FOR RELEASE SIMILAR TO WHAT IS RELEASED FROM } \\
\text { THE MAIN STACK. NOT IN OPERATION. }\end{array}$ & NONE & NONE & \\
\hline TAN & 868 & 001 & $\begin{array}{l}\text { VENT FOR TANKS USED FOR RECEIVING AND STORING LOW-LEVEL } \\
\text { RADIOACTIVE LIQUID WASTES. }\end{array}$ & NONE & NONE & \\
\hline$\overline{T A N}$ & 728 & 001 & RADIOACTIVE WATER STORAGE. VENTS FOR TANKS. & NONE & NONE & \\
\hline$\overline{\text { TAN }}$ & 734 & 001 & $\begin{array}{l}\text { MAIN EXHAUST STACK FOR THE TAN HOT CELL AND HOT CELL ANNEX } \\
\text { AREA }\end{array}$ & ONE TO THREE HEPA FILTERS IN SERIES & $89.97 \%$ & \\
\hline$\overline{\text { TAN }}$ & 607 & 136 & EQUIPMENT DECONTAMINATION ROOM ${ }^{\circ}$ & HEPA & $98.97 \%$ & \\
\hline TAN & & & OU 1-07B GROUNDWATER TREATMENT FACILITY & NONE & NONE & \\
\hline \multicolumn{7}{|c|}{ 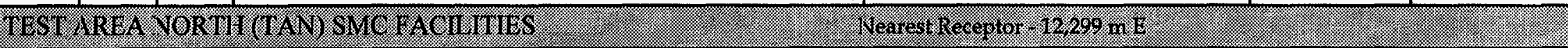 } \\
\hline TAN & 607 & 039 & RESEARCH AND DEVELOPMENT PROCESS STACK (RAD STACK \#1) & TWO HEPA FILTER BANKS & $99.97 \%$ & \\
\hline TAN & 607 & 119 & QUALITY CONTROL LAB (RAD STACK \#2) & TWO HEPA FILTER BANKS & $99.97 \%$ & \\
\hline TAN & 629 & 012 & MANUFACTURING PROCESS (RAD STACK \#5) & TWO HEPA FILTER BANKS & $99.97 \%$ & \\
\hline TAN & $\overline{629}$ & 013 & LINE 2A (RAD STACK \#3) - MANUFACTURING PROCESS & TWO HEPA FILTER BANKS & $98.97 \%$ & \\
\hline TAN & 628 & $\overline{014}$ & MANUFACTURING PROCESS (RAD STACK \#4) & TWO HEPA FILTER BANKS & $99.97 \%$ & \\
\hline$\overline{\text { TAN }}$ & 679 & 022 & $\begin{array}{l}\text { NORTH PROCESS (RAD STACK \#11) MANUFACTURING PROCESS } \\
\text { (EF-206) AND INCLUDES EMISSIONS FROM THE QC LAB }\end{array}$ & TWO HEPA FILTER BANKS & $89.97 \%$ & \\
\hline TAN & 679 & 023 & $\begin{array}{l}\text { NORTH PROCESS (RAD STACK \#10) MANUFACTURING PROCESS } \\
\text { (EF-205) AND INCLUDES EMISSIONS FROM THE QC LAB }\end{array}$ & TWO HEPA FILTER BANKS & $90.97 \%$ & \\
\hline TAN & 679 & 024 & $\begin{array}{l}\text { NORTH PROCESS (RAD STACK \#9)- MANUFACTURING PROCESS } \\
\text { (EF-204) AND INCLUDES EMISSIONS FROM THE QC LAB }\end{array}$ & TWO HEPA FILTER BANKS & $98.97 \%$ & \\
\hline TAN & 679 & 025 & SOUTH PROCESS (RAD STACK \#8) MANUFACTURING PROCESS (EF-203) & TWO HEPA FILTER BANKS & $99.97 \%$ & \\
\hline$\overline{\text { TAN }}$ & 679 & 026 & SOUTH PROCESS (RAD STACK \#7) MANUFACTURING PROCESS (EF-202) & TWO HEPA FILTER BANKS & $99.97 \%$ & \\
\hline$\overline{\text { TAN }}$ & 679 & 027 & SOUTH PROCESS (RAD STACK \#6) MANUFACTURING PROCESS (EF-201) & TWO HEPA FILTER BANKS & $99.97 \%$ & \\
\hline$\overline{\text { TAN }}$ & $\overline{681}$ & 012 & $\begin{array}{l}\text { PROCESS RECLAMATION FACILITY FOR NITRIC ACID WITH SCRUBBER } \\
\text { FOR NOx AND HEPA FILTERS FOR RAD (EF-209) (RAD STACK \#14) }\end{array}$ & $\begin{array}{l}\text { TWO HEPA FILTER BANKS } \\
\text { SCRUBBER }\end{array}$ & $\begin{array}{l}89.87 \% \\
50 \%\end{array}$ & \\
\hline$\overline{\text { TAN }}$ & 681 & 018 & $\begin{array}{l}\text { PROCESS RECLAMATION FACILITY INCLUDING DRYER HOOD, CALCINER } \\
\text { HOOD (RAD STACK\#13) }\end{array}$ & TWO HEPA FILTER BANKS & $99.97 \%$ & \\
\hline$\overline{\text { TAN }}$ & 681 & 020 & PROCESS RECLAMATION FACILITY (RAD STACK \#12) & TWO HEPA FILTER BANKS & $99.87 \%$ & \\
\hline
\end{tabular}

a. A single HEPA filter will remove $89.97 \%$ of particulates that are 0.3 microns in diameter.

b. Air emission sources that require continuous monitoring are based on an unabated emission potential of $0.1 \mathrm{mrem} / \mathrm{yr}$ or greater.

c. Not a source term for 1997 . 
Table II-7. TRA Radiological Air Emission Sources

\begin{tabular}{|c|c|c|c|c|c|c|}
\hline \multicolumn{4}{|c|}{ 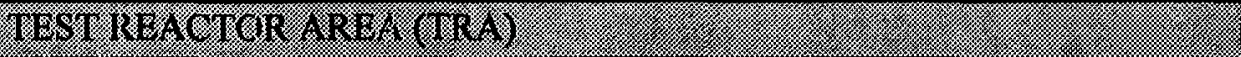 } & \multicolumn{3}{|l|}{ 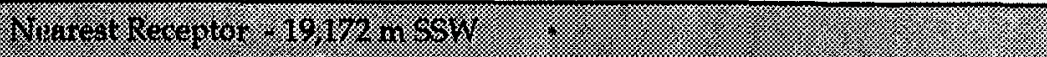 } \\
\hline (4) & 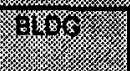 & \%"अ & 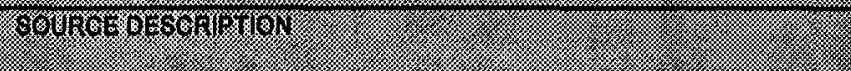 & Ty" & 9. & 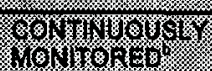 \\
\hline TRA & 604 & 035 & TRA RADIOCHEMISTRY LABORATORY FUMEHOOD EXHAUST (661/604) & HEPA FILTER & $99.97 \%$ & \\
\hline TRA & 604 & 072 & PERCHLORIC ACID HOOD EXHAUST IN 604 & NONE & NONE & \\
\hline TRA & .604 & 073 & PERCHLORIC ACID HOOD EXHAUST IN 604 & NONE & NONE & \\
\hline TRA & 604 & 074 & PERCHLORIC ACID HOOD EXHAUST IN 604 & NONE & NONE & \\
\hline TRA & 632 & 015 & DECONTAMINATION ROOM EXHAUST HOOD (not used during 1997) & HEPA FILTER & $99.97 \%$ & \\
\hline TRA & 632 & 018 & $\begin{array}{l}\text { HOT CELL USED FOR DECONTAMINATION OPERATIONS, } \\
\text { ASSEMBLING, DISASSEMBLING, OR DESTRUCTION OF RADIOACTIVE } \\
\text { MATERIALS }\end{array}$ & HEPA FILTER & $90.97 \%$ & \\
\hline TRA & 632 & 030 & $\begin{array}{l}\text { HOT CELL USED FOR DECONTAMINATION OPERATIONS, } \\
\text { ASSEMBLING, DISASSEMBLING, OR DESTRUCTION OF RADIOACTIVE } \\
\text { MATERIALS -ALSO METALLOGRAPHY }\end{array}$ & HEPA FILTER & $99.97 \%$ & \\
\hline TRA & 632 & 041 & $\begin{array}{l}\text { HOT CELL USED FOR DECONTAMINATION OPERATIONS, } \\
\text { ASSEMBLING, DISASSEMBLING, OR DESTRUCTION OF RADIOACTIVE } \\
\text { MATERIALS }\end{array}$ & HEPA FILTER & $99.97 \%$ & \\
\hline TRA & 680 & 004 & FUMEHOOD ${ }^{\circ}$ & HEPA FILTER & $99.97 \%$ & \\
\hline TRA & 681 & 008 & $\begin{array}{l}\text { VENTS THE NEW RADIOCHEMISTRY WING EXTENSION (7 HOODS AND } \\
2 \text { STORE ROOMS) }\end{array}$ & HEPA FILTER & $99.97 \%$ & \\
\hline TRA & 668 & 013 & LABORATORY 98 FUMEHOOD EXHAUST ${ }^{\circ}$ & NONE & NONE & \\
\hline TRA & 668 & 015 & LABORATORY 100 FUMEHOOD EXHAUST ${ }^{\circ}$ & NONE & NONE & \\
\hline$\overline{T R A}$ & 670 & 074 & $\begin{array}{l}\text { SAMPLE HOOD FOR PRIMARY AND SECONDARY COOLING WATER IN } \\
\text { ADVANCED TEST REACTOR (ATR) }\end{array}$ & HEPA FILTER & $89.97 \%$ & \\
\hline TRA & 670 & 086 & LABORATORY 131 FUMEHOOD EXHAUST & HEPA FILTER & $99.97 \%$ & \\
\hline$\overline{T R A}$ & 670 & 098 & $\begin{array}{l}\text { EXHAUST FROM TWO WET CHEMISTRY HOODS AND THE HEALTH } \\
\text { PHYSICS OFFICE AT ATR }\end{array}$ & HEPA FILTER & $99.97 \%$ & \\
\hline TRA & 710 & 001 & $\begin{array}{l}\text { MATERIAL TEST REACTOR (MTR) MAIN STACK (REACTOR SHUT } \\
\text { DOWN). THE TRA TRITIUM LABORATORY, CATCH TANK VENT (FROM } \\
\text { 604, 661, 632 AREAS), TRA-604/661 LAB HOT CELL VENT SCRUBBER, } \\
\text { AND TRA-605 BUILDING EXHAUST VENT OUT THIS STACK. }\end{array}$ & $\begin{array}{l}\text { TRA-604 - } 661 \text { HOT CELL VENT SCRUBBER } \\
\text { IS VENTED THROUGH A HEPA FILTER. ALL } \\
\text { OTHER SOURCES LISTED HAVE ONE HEPA } \\
\text { FILTER AND NONRADIOACTIVE SOURCES } \\
\text { HAVE NO EFFLUENT CONTROL. }\end{array}$ & $\begin{array}{l}99.97 \% \\
\text { each filter }\end{array}$ & \\
\hline TRA & 753 & 001 & $\begin{array}{l}\text { VENTILATION EXHAUST FROM ENGINEERING TEST REACTOR (ETR) } \\
\text { BULLING (SHUTDOWN) }\end{array}$ & NONE & NONE & 7 \\
\hline TRA & 770 & 001 & ATR MAIN STACK & NONE & NONE & \\
\hline
\end{tabular}

a. A single HEPA filter will remove $99.97 \%$ of partlculates that are 0.3 microns in diameter.

c. Air emission sources that require continuous monitoring are based on an unabated emission potential of 0.1 mrem/yr or greater. 


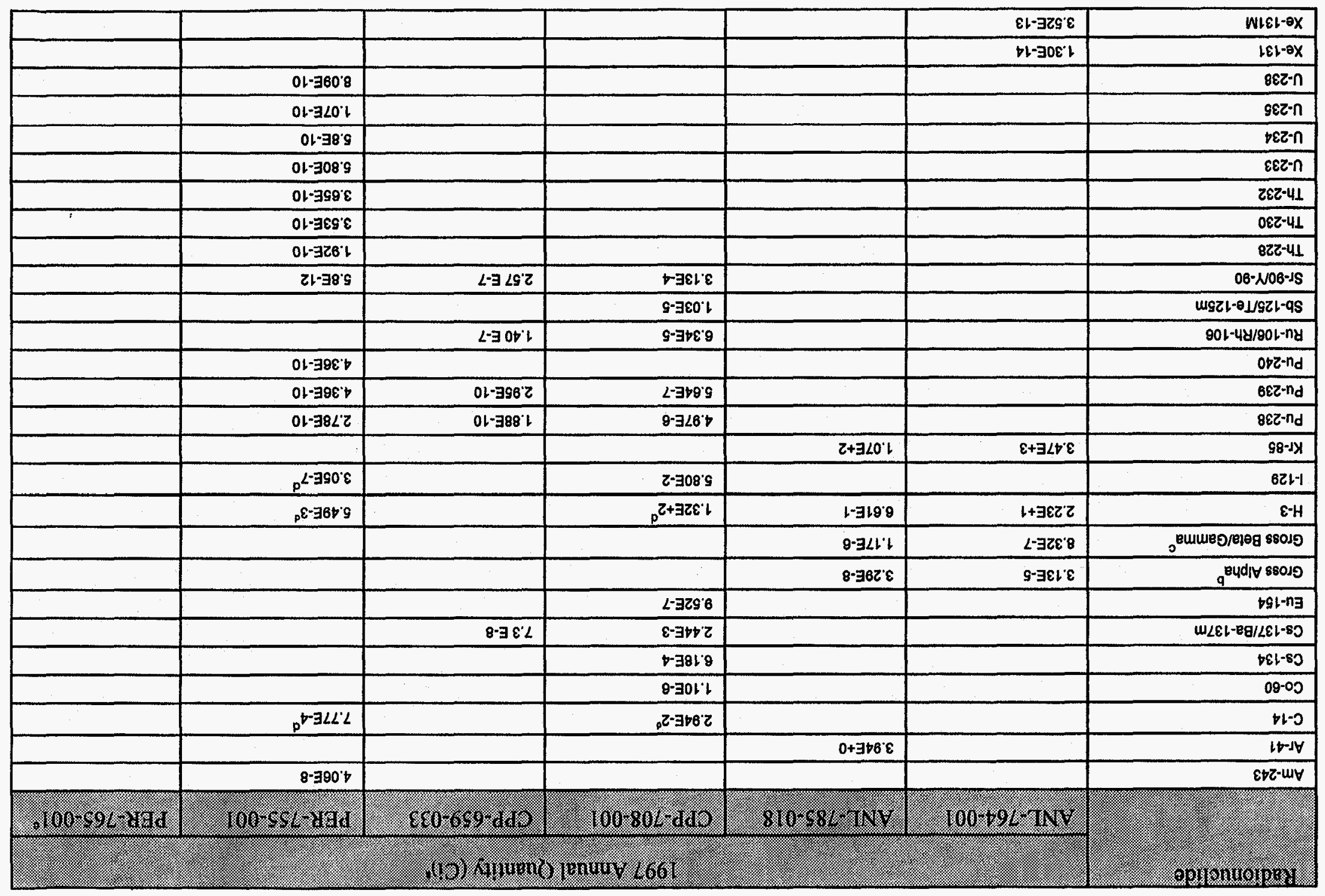


Table II-8.Point Source Radionuclides (Continuously Compliance Monitored Sources) From The INEEL Facilities During 1997 (Continued)
a. 1 Curie is equal to 37 Giga Becquerel
b. Assumed to be Pu-239
c. Assumed to be Sr-90/Y-90.
d. Emissions conservatively estimated based on engineering calculations; these specific nuclldes do not constitute $>10 \%$ of the potentlal effective dose equivalent.
e. No emissions reported for Stack 765 in 1997 
Table II-9. Point Source Radionuclides (All Other Release Points) From The INEEL Facilities During 1997

\begin{tabular}{|c|c|c|c|c|c|c|c|c|}
\hline \multirow{2}{*}{ 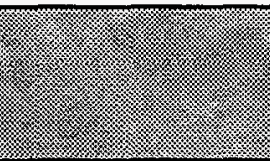 } & \multicolumn{8}{|c|}{ 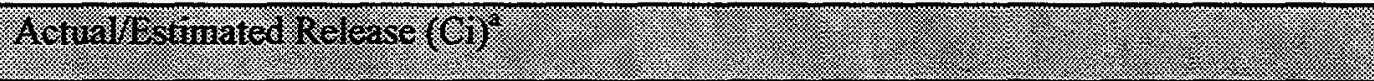 } \\
\hline & 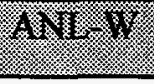 & (4) & (19P: & 19r: & PBF & Plip & 194 & T/24: \\
\hline Ac-228 & & $1.5 \mathrm{E}-15$ & & & & & & \\
\hline Am-241 & & $2.7 \mathrm{E}-13$ & $9.1 \mathrm{E}-7$ & & & & & $4.39 \mathrm{E}-11$ \\
\hline \multicolumn{9}{|l|}{ Am-243 } \\
\hline Ast-41 & & & & & & & & $1.55 \mathrm{E}+3$ \\
\hline Ba-139 & & & & & & & & $9.49 E-4$ \\
\hline Ba-140 & & & & & & & & $1.78 E-10$ \\
\hline$\overline{c-14}$ & & & & $8.1 E-1$ & & $1.4 \mathrm{E}-3$ & & \\
\hline Ce-141 & & & & & & & & $2.37 E-10$ \\
\hline Ce-144 & & & $4.9 \mathrm{E}-9$ & & & & & \\
\hline $60-57$ & & $3.0 \mathrm{E}-14$ & & & & & & \\
\hline Co-58 & & & & $8.7 E-8$ & & & & $4.00 E-7$ \\
\hline Co-60 & & $3.0 \mathrm{E}-14$ & $1.4 \mathrm{E}-5$ & $1.8 \mathrm{E}-6$ & $2.0 \mathrm{E}-8$ & & $2.5 E-7$ & $6.62 \mathrm{E}-4$ \\
\hline$\overline{C r-51}$ & & & & & & & & $5.60 \mathrm{E}-3$ \\
\hline Cs-134 & & & $5.7 E-9$ & & & & & $1.16 \mathrm{E}-6$ \\
\hline Cs-137/Ba-137m & & $8.5 E-13$ & $4.4 E-3$ & $3.6 \mathrm{E}-5$ & & & $1.3 E-7$ & $5.66 \mathrm{E}-6$ \\
\hline Cs-138 & & & & & & & & $6.86 E-2$ \\
\hline Eu-152 & & $5.3 \mathrm{E}-7$ & $2.2-E-5$ & & & & & $6.85 \mathrm{E}-7$ \\
\hline Eu-154 & & $1.5 \mathrm{E}-14$ & $1.1 \mathrm{E}-5$ & & & & & $1.48 \mathrm{E}-6$ \\
\hline Eu-155 & & & $3.5 E-8$ & & & & & $8.44 \mathrm{E}-7$ \\
\hline Fe-55 & & $1.1 E-12$ & & & & & & \\
\hline Gd-153 & & & & & & & & $5.80 E-6$ \\
\hline Gross Alpha ${ }^{b}$ & $1.08 \mathrm{E}-7$ & & & $3.9 E-6$ & $1.61 \mathrm{E}-8$ & & & $5.1 E-6$ \\
\hline 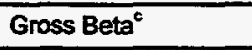 & & & & $6.9 \mathrm{E}-5$ & $1.72 E-7$ & & $5.9 E-7$ & $7.27 E-4$ \\
\hline Gross Beta/Gamma & $7.42 \mathrm{E}-6$ & & & & & & & \\
\hline $\mathrm{H}-3$ & $1.37 \mathrm{E}-2$ & $1.9 \mathrm{E}-11$ & $1.2 E-8$ & $7.8 \mathrm{E}-2$ & & $4.12 E-2$ & $2.1 \mathrm{E}-4$ & $1.29 \mathrm{E}+1$ \\
\hline $\mathrm{HF}-181$ & & & & & & & & $7.86 E-11$ \\
\hline $\mathrm{Hg}-203$ & & & & $3.0 E-7$ & & & & $1.67 E-4$ \\
\hline-129 & & & $1.8 \mathrm{E}-9$ & & & & & \\
\hline $1-131$ & & & & $1.7 E-5$ & & & & $5.54 E-4$ \\
\hline $1-132$ & & & & & & & & $5.40 E-4$ \\
\hline-133 & & & & & & & & $2.50 E-4$ \\
\hline It-192 & & & & & & & & $5.98 E-08$ \\
\hline$K-40$ & & $4.0 E-15$ & & & & & & \\
\hline $\mathrm{Kr}-85$ & $1.14 \mathrm{E}+0$ & & & & & & & \\
\hline $\mathrm{Kr}-85 \mathrm{~m}$ & & & & & & & & $3.01 \mathrm{E}+0$ \\
\hline $\mathrm{K}_{\mathrm{f}}-87$ & & & & & & & & $1.84 E+0$ \\
\hline $\mathrm{Kr}-88$ & & & & & & & & $3.54 \mathrm{E}+0$ \\
\hline Mn-54 & & $2.5 E-13$ & & & & & & $3.26 \mathrm{E}-11$ \\
\hline $\mathrm{Na-24}$ & & & & & & & & $1.42 E-02$ \\
\hline $\mathrm{Nb}-95$ & & & $3.0 \mathrm{E}-11$ & & & & & $3.05 E-11$ \\
\hline Np-237 & & $5.3 \mathrm{E}-8$ & $1.0 \mathrm{E}-04$ & & & & & \\
\hline Np-239 & & & & & & & & $7.88 E-10$ \\
\hline Os-191 & & & & $1.5 \mathrm{E}-5$ & & & & \\
\hline Pa-234/Pa-234m & & & $1.7 \mathrm{E}-7$ & & & & $4.2 \mathrm{E}-7$ & \\
\hline $\mathrm{Pr}-144$ & & & $4.9 E-9$ & & & & & \\
\hline Pu-238 & & $1.0 E-17$ & $1.1 \mathrm{E}-7$ & & & & & \\
\hline Pu-239 & $1.08 \mathrm{E}-7$ & $8.5 E-18$ & $4.1 E-7$ & & & $7.3 E-10$ & & $5.17 \mathrm{E}-7$ \\
\hline
\end{tabular}


Table II-9.Point Source Radionuclides (All Other Release Points) From The INEEL Facilities During 1997 (continued)

\begin{tabular}{|c|c|c|c|c|c|c|c|c|}
\hline & \multicolumn{8}{|c|}{ 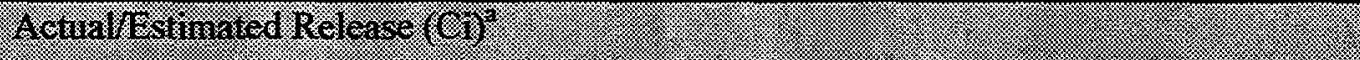 } \\
\hline & 4 & 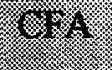 & $3 y^{380}$ & IIPI & 961 & 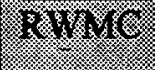 & 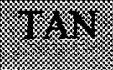 & $2 \pi$ \\
\hline Pu-240 & & $8.5 E-18$ & & & & & & \\
\hline $\begin{array}{l}\text { Pu-242 } \\
\text { Pu }\end{array}$ & & 2.7E-13 & & & & & & \\
\hline Ra-224 & & 1.5E-15 & & & & & & \\
\hline Ra-226 & & $2.1 \mathrm{E}-14$ & & & & & & \\
\hline $\operatorname{Ra}-228$ & & $3.1 E-15$ & & & & & & \\
\hline $\mathrm{Rb}-88$ & & & & & & & & $1.31 E+00$ \\
\hline $\mathrm{Rb}-89=$ & & & & & & & & $1.13 \mathrm{E}-2$ \\
\hline Re-186 & & & & & & & & $1.47 \mathrm{E}-9$ \\
\hline Ru-103 & & & & & & & & $9.99 \mathrm{E}-12$ \\
\hline Ru-106/Rh-106 & & & $1.4 \mathrm{E}-7$ & & & & & \\
\hline$S b-124$ & & & & & & & & $6.04 E-12$ \\
\hline Sb-125/Te-125m & & & $1.4 E-5$ & & & & & $2.63 E-6$ \\
\hline Sm-153 & & & & & & & & $9.62 \mathrm{E}-10$ \\
\hline ST-90/N-90 & & $2.2 E-12$ & $3.8 E-4$ & & & $6.8 \mathrm{E}-9$ & & $1.09 \mathrm{E}-6$ \\
\hline Ta-99m & & & & & & & & $2.23 E-3$ \\
\hline Th-228 & & $3.0 \mathrm{E}-15$ & & & & & & \\
\hline Th-230 & & $3.1 E-15$ & & & & & & \\
\hline Th-231 & & & $5.6 \mathrm{E}-12$ & & & & & \\
\hline Th-232 & & $1.5 \mathrm{E}-15$ & & & & & & \\
\hline Th-234 & & & $1.7 E-7$ & & & & $4.2 E-7$ & \\
\hline U-233 & & & $4.2 E-8$ & $\cdots$ & & & & \\
\hline U-234 & & $7.1 E-16$ & $8.9 E-7$ & & & & $5.4 E-8$ & \\
\hline U-235 & & $7.1 E-16$ & $1.1 \mathrm{E}-8$ & & & & $5.0 E-9$ & \\
\hline U-236 & & & $1.8 \mathrm{E}-9$ & & & & & 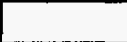 \\
\hline U-238 & & $2.6 \mathrm{E}-13$ & $1.7 E-7$ & & & & $4.2 \mathrm{E}-7$ & \\
\hline W-187 & & & & & & & & $7.07 E-09$ \\
\hline$x e-133$ & & & & & & & & $3.44 E+00$ \\
\hline$x e-135$ & & & & & & & & $2.09 \mathrm{E}+01$ \\
\hline Xe-135m & & & & & & & & $1.12 E+00$ \\
\hline$x \in-138$ & & & & & & & & $7.60 E-1$ \\
\hline$Y-90 m$ & & & & & & & & $7.57 E-4$ \\
\hline $2 n-65$ & & $7.7 E-14$ & & & & & & $6.40 \mathrm{E}-7$ \\
\hline$Z r-95$ & & & 3.0E-11 & & & & & \\
\hline
\end{tabular}

a. 1 Curie is equal to 37 Giga Becquerel.

b. Assumed to be Pu-239.

c. Assumed to be Sr-90r-90.

d. NRF point source emissions include non-point source (diffuse) emissions. 
Table II-10. Non-Point Source Radionuclides (Diffuse Emission Sources) From The INEEL Facilities During $1997^{\mathrm{a}}$

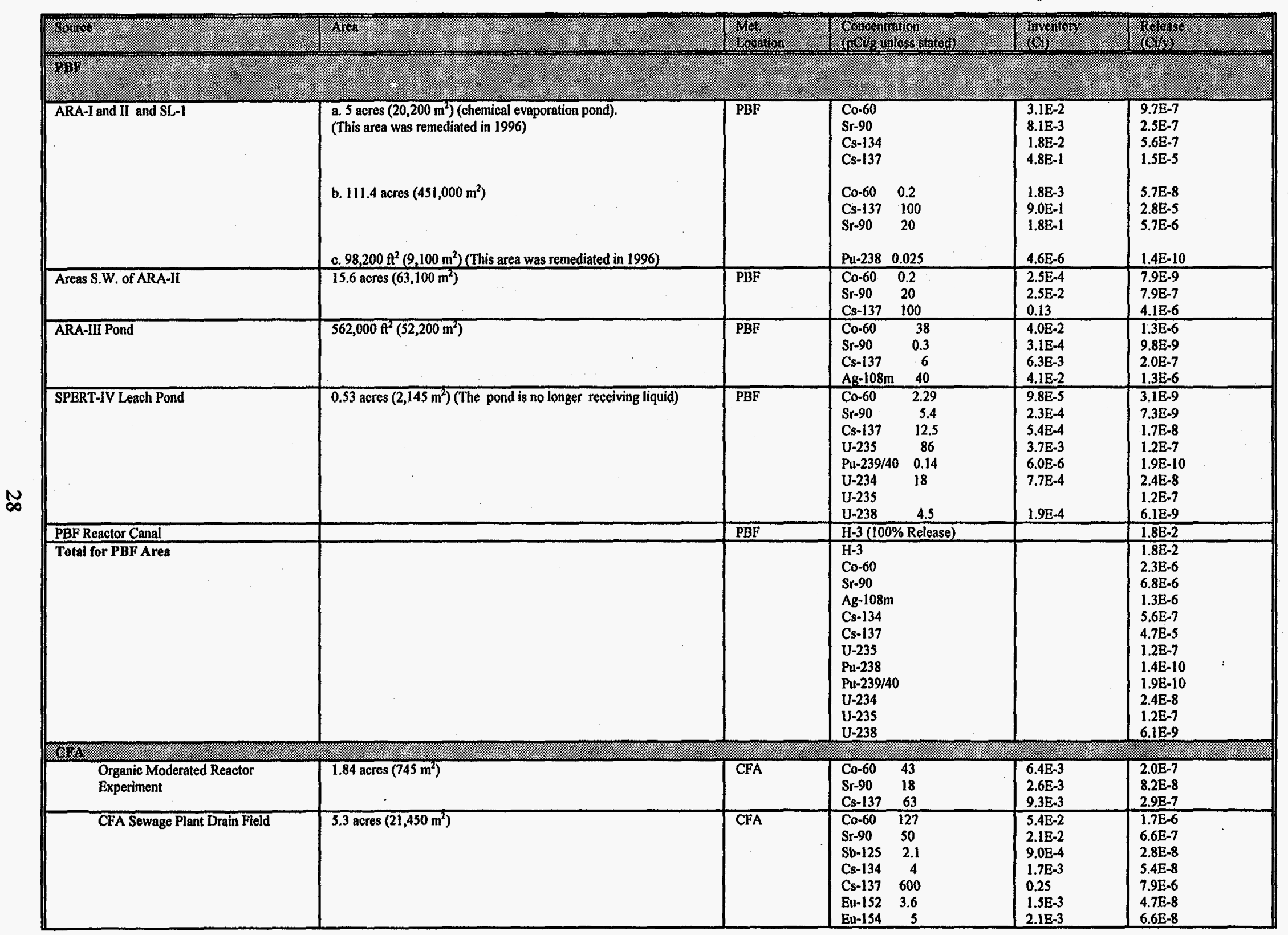


Table II-10. Non-Point Source Radionuclides (Diffuse Emission Sources) From The INEEL Facilities During $1997^{\mathrm{a}}$ (continued)

\begin{tabular}{|c|c|c|c|c|c|}
\hline 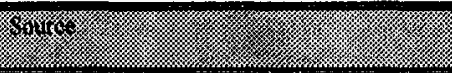 & 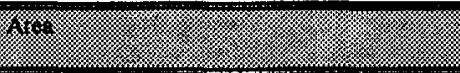 & mexis: & 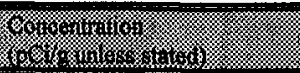 & 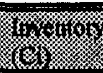 & feriso \\
\hline & & & $\begin{array}{ll}\text { Eu-155 } & 1.6 \\
\text { U-235 } & 0.01 \\
\end{array}$ & $\begin{array}{l}6.9 \mathrm{E}-4 \\
4.3 \mathrm{E}-6\end{array}$ & $\begin{array}{l}2.2 \mathrm{E}-8 \\
1.4 \mathrm{E}-10\end{array}$ \\
\hline $\begin{array}{l}\text { CFA Tritium to air from Misc. } \\
\text { aquifer water use }\end{array}$ & $\begin{array}{l}1,0 \text { acre }\left(4,047 \mathrm{~m}^{2}\right) \\
\text { (conservative assumption) }\end{array}$ & CFA & $\begin{array}{l}\mathrm{H}-3 \text { (present in aquifer water } \\
\text { pumped and used at CFA) }\end{array}$ & 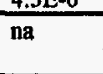 & $\frac{\frac{1.4 \mathrm{E}-1 \mathrm{~V}}{2.2 \mathrm{E}+0}}{2}$ \\
\hline CFA Ditch and Pit & 1.0 acre $\left(4,047 \mathrm{~m}^{2}\right)$ & CFA & $\begin{array}{lr}\text { Co-60 } & 26.4 \\
\text { Cs-134 } & 0.9 \\
\text { Cs-137 } & 2988.0 \\
\text { Eu-154 } & 9.9\end{array}$ & $\begin{array}{l}2.1 \mathrm{E}-3 \\
7.3 \mathrm{E}-5 \\
2.4 \mathrm{E}-1 \\
8.0 \mathrm{E}-4\end{array}$ & $\begin{array}{l}6.6 \mathrm{E}-8 \\
2.3 \mathrm{E}-9 \\
7.6 \mathrm{E}-6 \\
2.5 \mathrm{E}-8\end{array}$ \\
\hline Total for CFA Area & & & $\begin{array}{l}H-3 \\
\text { Co-60 } \\
\text { Sr-90 } \\
\text { Sb-125 } \\
\text { Cs-134 } \\
\text { Cs-137 } \\
\text { Eu-152 } \\
\text { Eur-154 } \\
\text { Eu-155 } \\
\text { U-235 } \\
\end{array}$ & & $\begin{array}{l}2.2 \mathrm{E}+0 \\
2.0 \mathrm{E}-6 \\
7.4 \mathrm{E}-7 \\
2.8 \mathrm{E}-8 \\
5 \mathrm{E}-8 \\
1.6 \mathrm{E}-5 \\
4.7 \mathrm{E}-8 \\
9.1 \mathrm{E}-8 \\
2.2 \mathrm{E}-8 \\
1.4 \mathrm{E}-10\end{array}$ \\
\hline 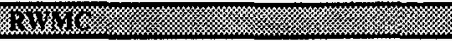 & & & & & \\
\hline EBR-I & a. 2.2 acres $\left(8,903 \mathrm{~m}^{2}\right)$ & CFA & $\begin{array}{ll}\text { Css-137 } & 108 \\
\text { Sr } 90 & 2.9\end{array}$ & $\begin{array}{l}1.9 \mathrm{E}-2 \\
5.2 \mathrm{E}-4\end{array}$ & $\begin{array}{l}6.0 \mathrm{E}-7 \\
1.6 \mathrm{E}-8\end{array}$ \\
\hline & b. 2.2 acres $\left(8,903 \mathrm{~m}^{2}\right)$ & & $\begin{array}{lr}\text { Cs-137 } 2090 \\
\text { Sro } 90\end{array}$ & 0.37 & $1.2 \mathrm{E}-5$ \\
\hline BORAX-I & $\begin{array}{l}0.64 \text { acres }\left(2,590 \mathrm{~m}^{2}\right) \\
\text { This site was remediated in } 1996)\end{array}$ & CFA & $\begin{array}{ll}\text { Cs.137 } & 227 \\
\mathrm{U}-235 & 5.82\end{array}$ & $\begin{array}{l}1.2 \mathrm{E}-2 \\
3.0 \mathrm{E}-4\end{array}$ & $\begin{array}{l}3.7 \mathrm{E}-7 \\
9.5 \mathrm{E}-9\end{array}$ \\
\hline $\begin{array}{l}\text { SDA Gaseous Releases from Buried } \\
\text { Waste }\end{array}$ & Treat as a point source at Pit 17 (conservative) & CFA & $\begin{array}{l}\mathrm{H}-3 \\
\mathrm{C}-14\end{array}$ & & $\begin{array}{l}1.6 \mathrm{E}+2 \\
6.8 \mathrm{E}-2\end{array}$ \\
\hline Total for RWMC Area & & & $\begin{array}{l}\mathrm{H}-3 \\
\text { C-14 } \\
\text { Cs-137 } \\
\text { Sr-90 } \\
\text { U-235 }\end{array}$ & & $\begin{array}{l}1.6 \mathrm{E}+2 \\
6.8 \mathrm{E}-2 \\
1.4 \mathrm{E}-5 \\
3.0 \mathrm{E}-8 \\
9.5 \mathrm{E}-9\end{array}$ \\
\hline
\end{tabular}


Table II-10. Non-Point Source Radionuclides (Diffuse Emission Sources) From The INEEL Facilities During $1997^{\mathrm{a}}$ (continued)

\begin{tabular}{|c|c|c|c|c|c|c|}
\hline \%垃- & 8 & 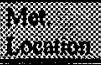 & \multicolumn{2}{|c|}{ (colocinininis. } & 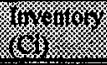 & 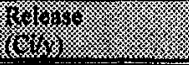 \\
\hline \multicolumn{7}{|l|}{ (6) \% } \\
\hline \multirow[t]{21}{*}{ ICPP Percolation Pond (Dry) } & \multirow[t]{21}{*}{$4.47 \mathrm{E}+05 \mathrm{ft}^{2}\left(41,509 \mathrm{~m}^{2}\right)$} & GRD3 & \multicolumn{2}{|c|}{$\mathrm{Am}-241 \quad 0.15$} & $7.0 \mathrm{E}-5$ & \multirow{17}{*}{$\begin{array}{l}2.2 \mathrm{E}-9 \\
8.8 \mathrm{E}-9 \\
9.2 \mathrm{E}-9 \\
2.6 \mathrm{E}-8 \\
6.9 \mathrm{E}-7 \\
4.5 \mathrm{E}-9 \\
3.8 \mathrm{E}-8 \\
1.6 \mathrm{E}-8 \\
1.3 \mathrm{E}-8 \\
4.0 \mathrm{E}-9 \\
{[1.7 \mathrm{E}-8 \text { total Pu] }} \\
4.5 \mathrm{E}-8 \\
4.5 \mathrm{E}-8 \\
9.5 \mathrm{E}-9 \\
9.5 \mathrm{E}-9 \\
8.9 \mathrm{E}-9\end{array}$} \\
\hline & & & $\mathrm{Sb}-125$ & 0.60 & 2.8E-4 & \\
\hline & & & Ce-144 & 0.63 & $2.9 \mathrm{E}-4$ & \\
\hline & & & Cs-134 & 1.75 & $8.1 \mathrm{E}-4$ & \\
\hline & & & Cs-137 & 46.8 & $2.2 \mathrm{E}-2$ & \\
\hline & & & Co-60 & 0.31 & $1.4 \mathrm{E}-4$ & \\
\hline & & & $\mathrm{I}-129$ & 2.6 & $1.2 \mathrm{E}-3$ & \\
\hline & & & $\mathrm{Np}-237$ & 1.1 & $5.1 \mathrm{E}-4$ & \\
\hline & & & Pu-238 & 0.9 & $4.2 \mathrm{E}-4$ & \\
\hline & & & \multirow{3}{*}{\multicolumn{2}{|c|}{$\begin{array}{l}\text { [all Pu modeled as Pu-238] } \\
\text { Ru-106 3.1 }\end{array}$}} & $1.3 \mathrm{E}-4$ & \\
\hline & & & & & & \\
\hline & & & & & 1 & \\
\hline & & & Rh-106 & 3.1 & $1.4 E_{-3}$ & \\
\hline & & & & 0.65 & $3.0 \mathrm{E}-4$ & \\
\hline & & & H-3 & 0.61 & $3.0 \mathrm{E}-4$ & \\
\hline & & & \multirow{2}{*}{\multicolumn{2}{|c|}{ [assume $\mathrm{H}-3$ in dry area is a }} & $2.8 \mathrm{E}-4$ & \\
\hline & & & & & & \\
\hline & & & \multirow{2}{*}{\multicolumn{2}{|c|}{$\begin{array}{ll}\text { solid form] } & \\
\text { U-234 } & 0.77 \\
\text { U-235 } & 0.07\end{array}$}} & & $1.1 \mathrm{E}-8$ \\
\hline & & & & & $3.6 \mathrm{E}-4$ & $1.0 \mathrm{E}-9$ \\
\hline & & & \multirow{2}{*}{\multicolumn{2}{|c|}{$\begin{array}{l}\text { U-238 } 0.82 \\
\text { [all U modeled as U-234] }\end{array}$}} & 3.3E-5 & $1.2 \mathrm{E}-8$ \\
\hline & & & & & $3.8 \mathrm{E}-4$ & {$[2.4 \mathrm{E}-8$ total U] } \\
\hline \multirow[t]{6}{*}{ Inside ICPP Fence } & \multirow[t]{6}{*}{150 acres $\left(6.07 \mathrm{E}+05 \mathrm{~m}^{2}\right)$} & GRD3 & Cs-137 & 12.0 & $1.5 \mathrm{E}-1$ & $4.6 \mathrm{E}-6$ \\
\hline & & & Eu-152 & & $3.6 \mathrm{E}-2$ & $1.1 \mathrm{E}-6$ \\
\hline & & & $\mathrm{Nb}-95$ & 0.08 & $9.7 \mathrm{E}-4$ & $3.1 \mathrm{E}-8$ \\
\hline & & & Ru-106 & 0.07 & $8.5 \mathrm{E}-4$ & $2.7 \mathrm{E}-8$ \\
\hline & & & Rh-106 & 0.07 & 8.5E-4 & $2.7 \mathrm{E}-8$ \\
\hline & & & $\mathrm{Sb}-125$ & 0.6 & 7.3E-3 & $2.3 \mathrm{E}-7$ \\
\hline Outside ICPP Fence & 19.2 acres $\left(77,700 \mathrm{~m}^{2}\right)$ & GRD3 & \multicolumn{2}{|c|}{$\begin{array}{ll}\text { Cs-137 } & 46.1 \\
\end{array}$} & $7.2 \mathrm{E}-2$ & $2.3 \mathrm{E}-6$ \\
\hline \multirow[t]{18}{*}{ Total for ICPP Area } & & GRD3 & \multirow{18}{*}{\multicolumn{2}{|c|}{$\begin{array}{l}\text { Am-241 } \\
\text { Sb-125 } \\
\text { Ce-144 } \\
\text { Cs-134 } \\
\text { Cs-137 } \\
\text { Co-60 } \\
\text { I-129 } \\
\text { Np-237 } \\
\text { Pu-238 } \\
\text { Pu-239 } \\
\text { [all Pu modeled as Pu-238] } \\
\text { Ru-106 } \\
\text { Rh-106 } \\
\text { Sr-90 } \\
\text { - }-90 \\
\text { H-3 } \\
\text { U-234 } \\
\text { U-235 } \\
\text { U-238 } \\
\text { [all U modeled as U-234] } \\
\text { Nb-95 } \\
\text { Eu-152 }\end{array}$}} & & $2.2 \mathrm{E}-9$ \\
\hline & & & & & & $2.4 \mathrm{E}-7$ \\
\hline & & & & & & $\begin{array}{l}9.2 \mathrm{E}-9 \\
2.6 \mathrm{E}-8\end{array}$ \\
\hline & & & & & & $7.6 \mathrm{E}-6$ \\
\hline & & & & & & 4.5E-9 \\
\hline & & & & & & $3.8 \mathrm{E}-8$ \\
\hline & & & & & & $1.6 \mathrm{E}-8$ \\
\hline & & & & & & $1.3 \mathrm{E}-8$ \\
\hline & & & & & & [1.7E-8 total Pu] \\
\hline & & & & & & $7.2 \mathrm{E}-8$ \\
\hline & & & & & & $7.2 \mathrm{E}-8$ \\
\hline & & & & & & $9.5 \mathrm{E}-9$ \\
\hline & & & & & & $\begin{array}{l}9.5 \mathrm{E}-9 \\
8.9 \mathrm{E}-9\end{array}$ \\
\hline & & & & & & $1.1 \mathrm{E}-8$ \\
\hline & & & & & & $1.0 \mathrm{E}-9$ \\
\hline & & & & & & $1.2 \mathrm{E}-8$ \\
\hline & & & & & & {$[2.4 \mathrm{E}-8$ total $\mathrm{U}]$} \\
\hline & & & & & & $\begin{array}{l}3.1 \mathrm{E}-8 \\
1.1 \mathrm{E}-6\end{array}$ \\
\hline
\end{tabular}


Table II-10. Non-Point Source Radionuclides (Diffuse Emission Sources) From The INEEL Facilities During $1997^{\mathrm{a}}$ (continued)

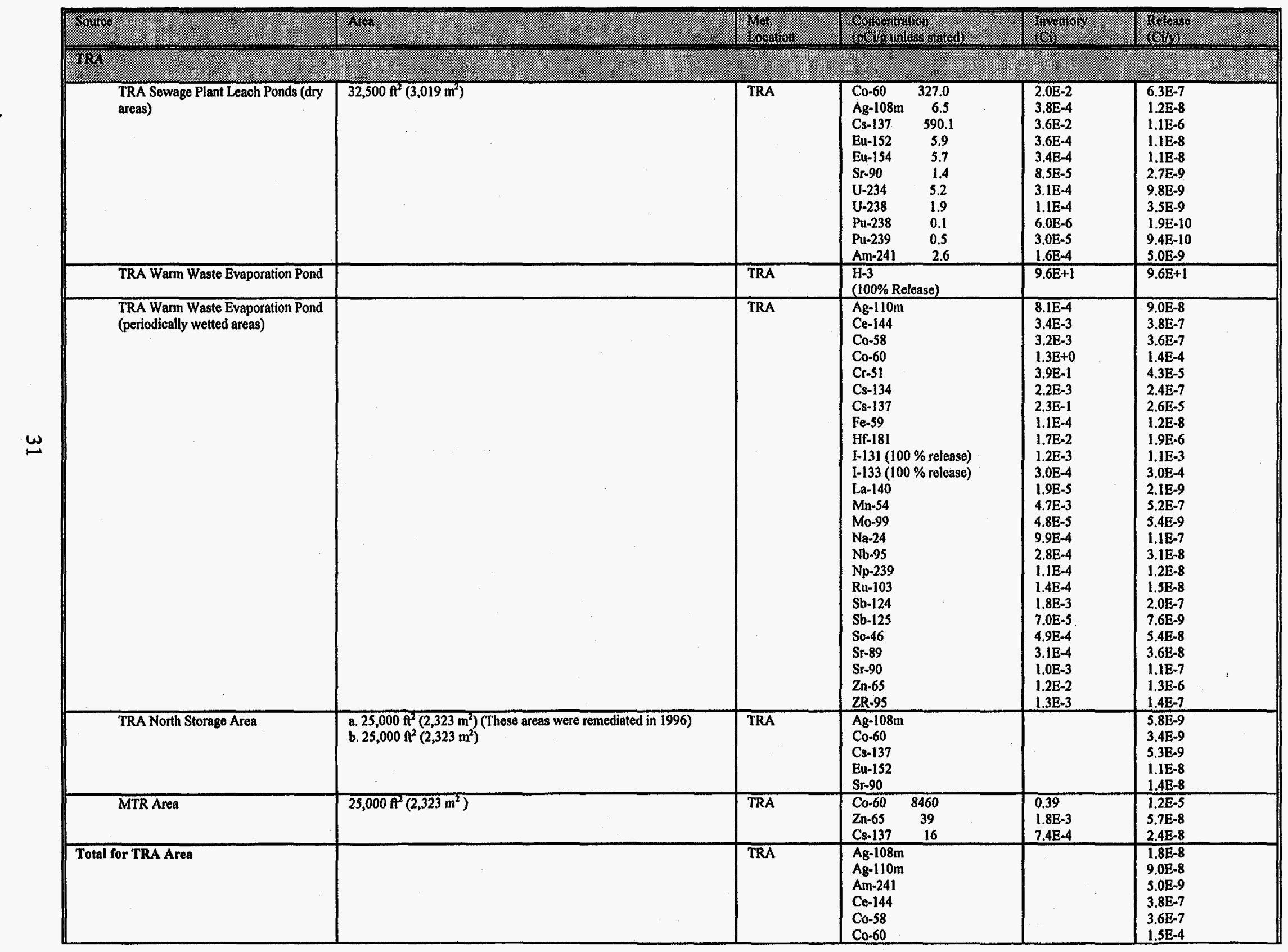


Table II-10. Non-Point Source Radionuclides (Diffuse Emission Sources) From The INEEL Facilities During $1997^{\mathrm{a}}$ (continued)

\begin{tabular}{|c|c|c|c|c|c|}
\hline 2.2 & reat & Mres: & 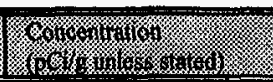 & 28 & Reterse \\
\hline & & & 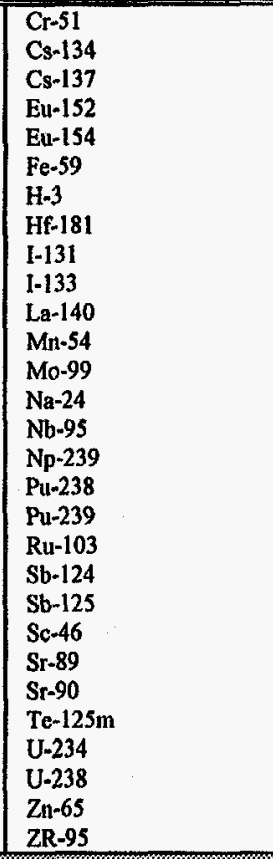 & & 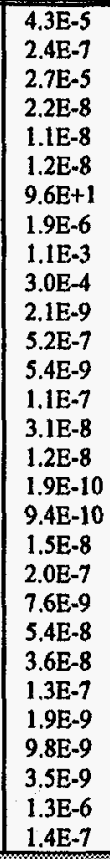 \\
\hline $\begin{array}{c}\text { TAN TSF-06, removal of soil from } \\
\text { areas } 7,8,9,11,11 \mathrm{~b}\end{array}$ & $670 \mathrm{yd}^{3}\left(513 \mathrm{~m}^{3}\right)$ & TAN & 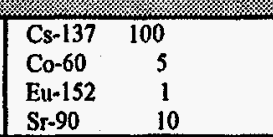 & & $\begin{array}{l}1.4 \mathrm{E}-5 \\
7.0 \mathrm{E}-7 \\
1.4 \mathrm{E}-7 \\
1.4 \mathrm{E}-6\end{array}$ \\
\hline TAN TSF-26. PM-2A Tank site-soil & $1,800 \mathrm{yd}^{3}\left(1,37 \mathrm{~m}^{3}\right)$ & TAN & \begin{tabular}{|l|l|} 
Co-60 & 5 \\
\end{tabular} & & $\frac{1.98-6}{1.10-4}$ \\
\hline $\begin{array}{l}\text { removal } \\
\text { TAN Radioactive Pants Storage } \\
\text { Security Area (RPSSA) }\end{array}$ & $600,000 \mathrm{f}^{2}\left(55,740 \mathrm{~m}^{2}\right)$ & TAN & 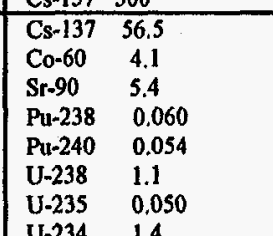 & 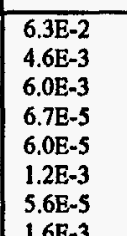 & 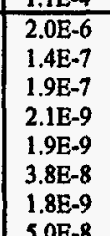 \\
\hline TAN V1, V2 and V3 Tank Area & $5,500 \mathrm{f}^{2}\left(511 \mathrm{~m}^{2}\right)$ & TAN & 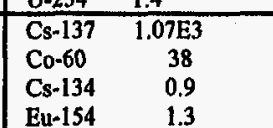 & 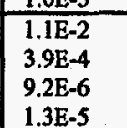 & $\begin{array}{l}3.5 \mathrm{SE-}-1 \\
1.2 \mathrm{E}-8 \\
2.9 \mathrm{E}-10 \\
4.1 \mathrm{E}-10\end{array}$ \\
\hline TANDisposal Pond - High Area & $\begin{array}{l}40,000 \mathrm{f}^{2} \\
\left(3,7116 \mathrm{~m}^{2}\right)\end{array}$ & TAN & \begin{tabular}{|ll}
$C s-137$ & 48 \\
Co -60 & 1.4 \\
\end{tabular} & $\begin{array}{l}3.56-3 \\
3.0 \mathrm{BE}-3 \\
1.0 \mathrm{E}-4\end{array}$ & $\begin{array}{l}\frac{1.10-10}{1.18-7} \\
3.2 \mathrm{E}-9\end{array}$ \\
\hline TANDisposal Pond-Depressions & $\begin{array}{l}8,000 \mathrm{n}^{2} \\
\left(743 \mathrm{~m}^{2}\right)\end{array}$ & TAN & 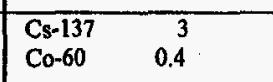 & $\begin{array}{l}\begin{array}{l}4.4 \mathrm{E}-5 \\
6.0 \mathrm{E}-6\end{array} \\
\end{array}$ & $\begin{array}{l}1.4 \mathrm{E}-9 \\
1.9 \mathrm{E}-10\end{array}$ \\
\hline
\end{tabular}


Table II-10. Non-Point Source Radionuclides (Diffuse Emission Sources) From The INEEL Facilities During $1997^{\mathrm{a}}$ (continued)

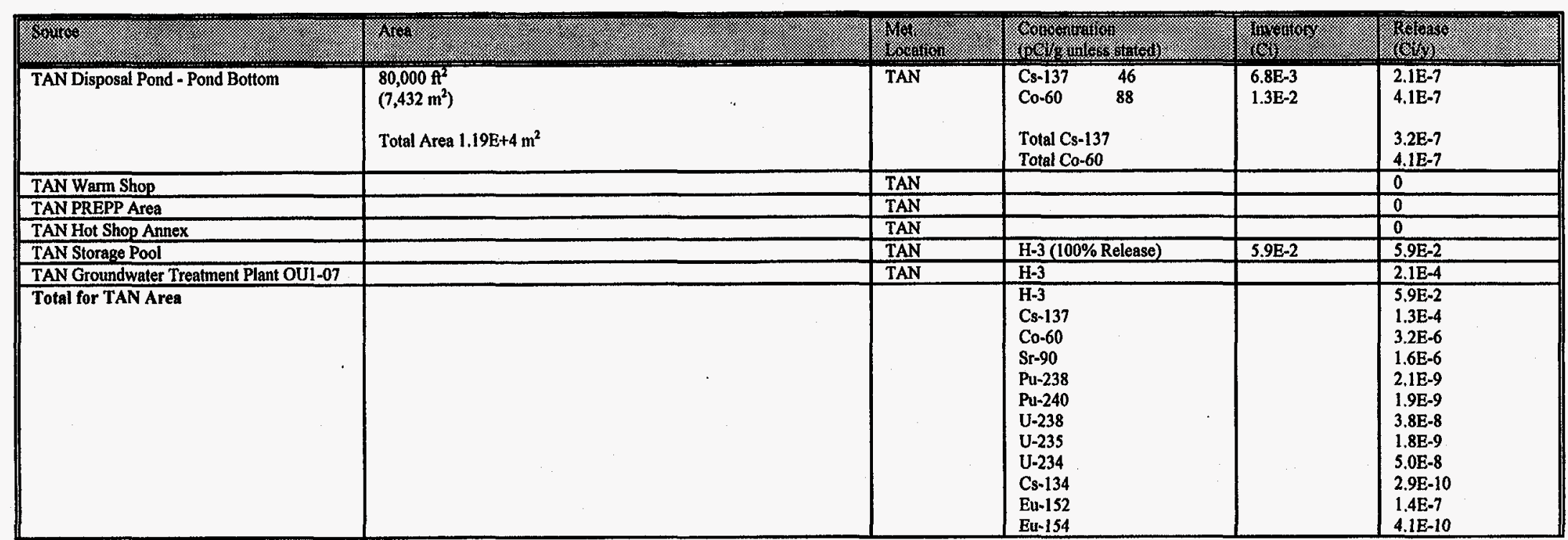

$\ddot{w}$

a. Diffuse emission sources for NRF are included in Table II-9 and are listed in Appendix A. 


\section{DOSE ASSESSMENTS}

\section{- Summary}

Tables III-1 and III-2 summarize the 1997 INEEL emission points for continuously compliance monitored sources and all other point sources, respectively, and the EDE associated with each. Table III-3 summarizes the 1997 diffuse emission sources at the INEEL, and the EDE associated with each. 40 CFR 61, Subpart H, requires that "compliance with the standard be determined by calculating the highest effective dose equivalent to any member of the public at any offsite point where there is a residence, school, business or office." The EDE to the MEI calculated for emissions from continuously compliance monitored release points during 1997 is $1.94 \mathrm{E}-02$ mrem (1.94E-07 Sievert). The EDE to the MEI for the remaining point sources is 4.09E-03 mrem (4.09E-08 Sievert). The diffuse source EDE to the MEI is 4.25E-03 mrem (4.25E-08 Sievert). These EDEs, when summed, result in a calculated 1997 total EDE to the MEI from the entire INEEL of 2.77E-02 mrem (2.77E-07 Sievert).

The following sections provide the methodology used to calculate radiological dose impacts, and the dose impacts associated with each INEEL operations area. 


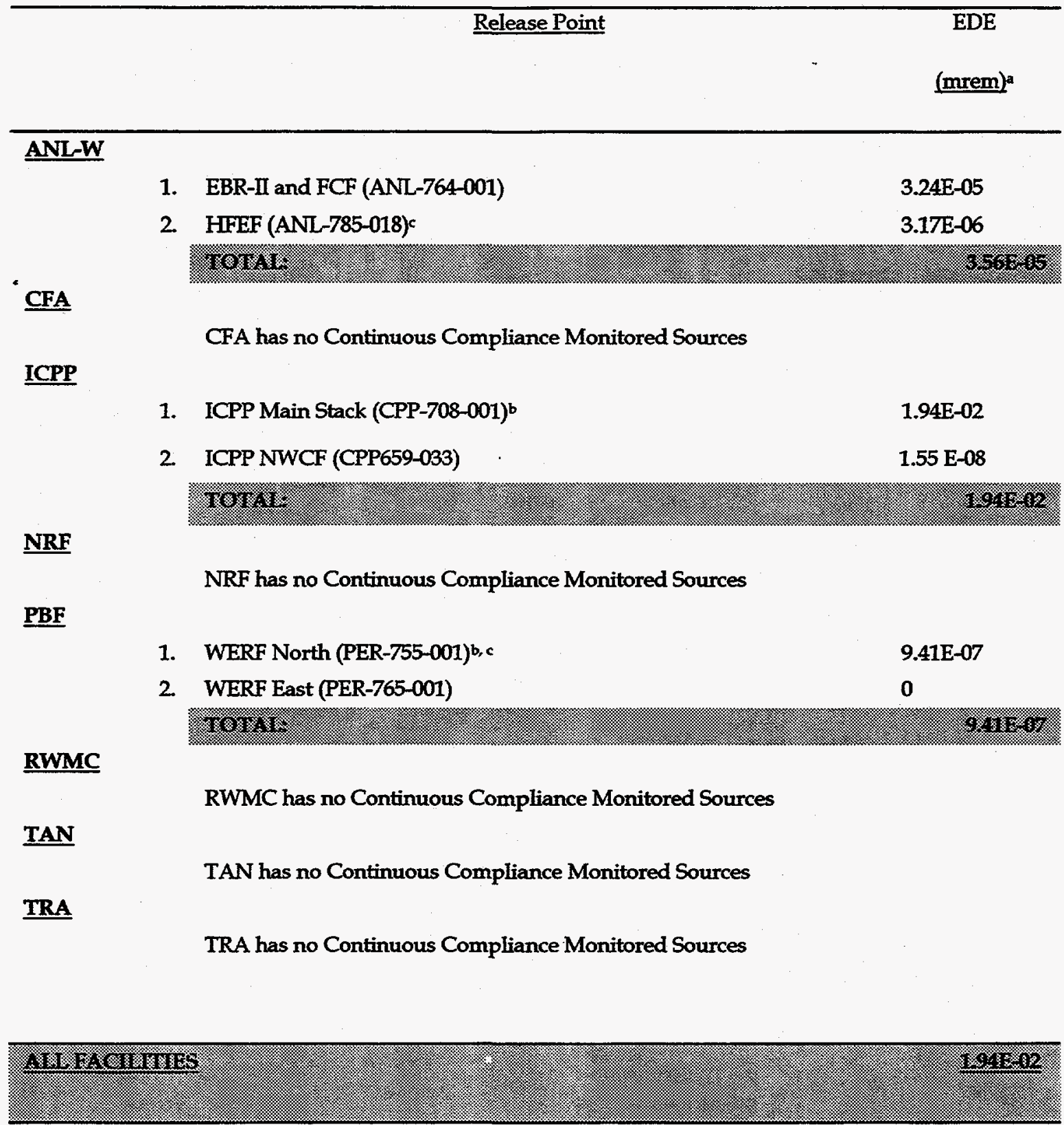
a. The EDE shown is to the INEEL MEI.
b. Includes dose from unmonitored gaseous emissions.
c. Includes stack releases being modeled as ground-level releases due to building wake effects 
Table III-2. Summary Of 1997 Effective Dose Equivalents From Other Release Points At The INEEL

\begin{tabular}{|c|c|c|}
\hline & Release Point & $\mathrm{EDE} \quad$ (mrem) $^{2}$ \\
\hline \multicolumn{3}{|l|}{ ANL-W } \\
\hline & ANL-W Ground-level releases ${ }^{b}$ & $2.17 \mathrm{E}-07$ \\
\hline & 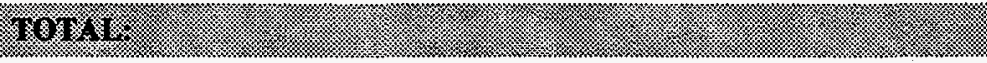 & (3) \\
\hline \multicolumn{3}{|l|}{ CFA } \\
\hline & RESL $^{\mathbf{b}}$ & $2.66 \mathrm{E}-12$ \\
\hline & 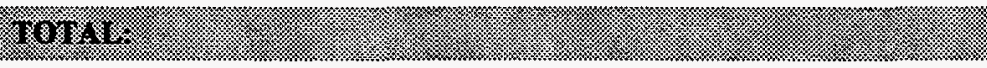 & 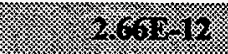 \\
\hline & \multicolumn{2}{|c|}{$\underline{\text { ICPP }}$} \\
\hline 1. & ICPP Ground-level releases ${ }^{b}$ & \multirow{2}{*}{$\begin{array}{l}6.21 \mathrm{E}-04 \\
8.07 \mathrm{E}-07\end{array}$} \\
\hline 2. & ICPP FAST Stack (CPP-767-001) & \\
\hline & (9014. & (3) \\
\hline \multicolumn{3}{|l|}{ NRF } \\
\hline & NRF (AIW, AlW-RWDS, ECF, S1W, S5G) ${ }^{6}$ & $4.31 \mathrm{E}-04$ \\
\hline & 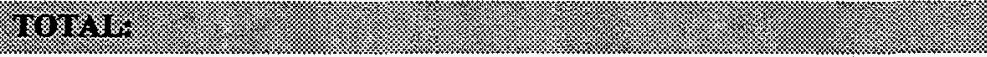 & (3) \\
\hline & \multicolumn{2}{|c|}{ PBF } \\
\hline & PBF (PER-620-016) ${ }^{b}$ & $3.90 \mathrm{E}-09$ \\
\hline & WERF South (PER-756-001) ${ }^{b}$ & 0 \\
\hline 3. & Mixed Waste Storage Facility - Repackaging Booth ${ }^{b}$ & $9.59 \mathrm{E}-09$ \\
\hline & 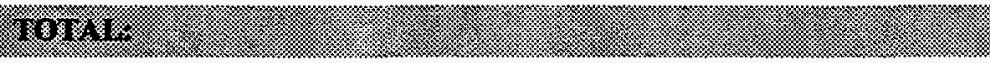 & 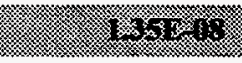 \\
\hline \multicolumn{3}{|l|}{ RWMC } \\
\hline 1. & OCVZ and Processing Tent ${ }^{b}$ & $3.94 \mathrm{E}-06$ \\
\hline & 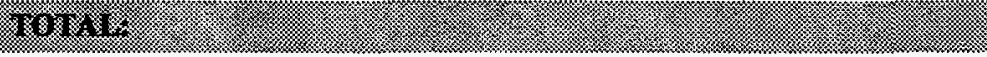 & (3) \\
\hline \multicolumn{3}{|l|}{ TAN } \\
\hline 1. & SMC (SMC Stacks S1-S14) ${ }^{b}$ & $5.70 \mathrm{E}-08$ \\
\hline 2. & TSF Exhaust (TAN-734-001) & $1.50 \mathrm{E}-08$ \\
\hline & Groundwater Treatment Unit (TAN OU 1-07B) ${ }^{b}$ & 6.37E-10 \\
\hline & TAN 666-001, Waste Tank Vent ${ }^{b}$ & $2.04 \mathrm{E}-12$ \\
\hline & 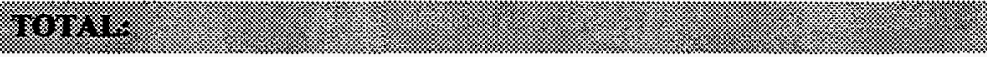 & (1) \\
\hline \multicolumn{3}{|c|}{ 然 } \\
\hline 1. & $\begin{array}{l}\text { MTR Fumehoods (TRA-604-035, }-072,-073,-074) \text {, TRA Hot Cell (TRA- } \\
632 \text { ), Chem. Lab. Addition (TRA-661-008), and ATR Laboritories (TRA- } \\
670-074,-086 \text {, and }-098)^{b}\end{array}$ & 3.63E-05 \\
\hline & MTR (TRA-710-001) & $3.49 E-07$ \\
\hline 3. & ATR (TRA-770-001) & $3.00 \mathrm{E}-03$ \\
\hline & ETR (TRA 753-001) & $1.03 \mathrm{E}-06$ \\
\hline & 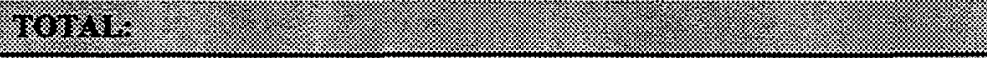 & (2) \\
\hline 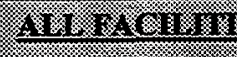 & & $\frac{1}{20}$ \\
\hline
\end{tabular}


Table III-3. Summary Of 1997 Effective Dose Equivalents From Diffuse Sources At The INEEL

\begin{tabular}{|c|c|}
\hline Release Area & $\begin{array}{c}\mathrm{EDE} \\
\text { (mrem) })^{\mathrm{a}}\end{array}$ \\
\hline ANL-W & 0 \\
\hline CFA & $1.53 \mathrm{E}-05$ \\
\hline ICPP & 4.75E-07 \\
\hline NRF & $7.66 \mathrm{E}-07$ \\
\hline PBF & $2.62 \mathrm{E}-06$ \\
\hline RWMC & $3.43 \mathrm{E}-03$ \\
\hline TAN & $1.61 \mathrm{E}-06$ \\
\hline TRA & $7.95 \mathrm{E}-04$ \\
\hline 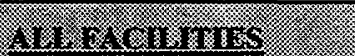 & 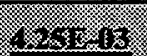 \\
\hline
\end{tabular}

a. The EDE shown is to the INEEL MEI. 


\section{Description of Dose Model and Summary of Input Parameters}

\section{General}

The CAP-88 computer code (EPA 1990) was used to calculate the EDE from the INEEL releases. CAP-88 is approved for use by the EPA for demonstrating compliance with 40 CFR 61, Subpart $H$. Because the MEI for emissions from the INEEL facilities is more than $3 \mathrm{~km}$ from all emission sources, the COMPLY code was not used. The output from CAP-88 is the EDE, which includes the 50-year committed EDE (CEDE) from internal exposure through the ingestion and inhalation pathways and the external EDE from ground deposition and air immersion. The dose conversion factors are from the RADRISK dosimetric data base.

Site-specific 1997 wind data collected by the National Oceanic and Atmospheric Administration (NOAA) were used as input to the CAP-88 computer code (see Appendix C), with calm wind periods incorporated into the lowest windspeed class. Most INEEL facilities have NOAA stations onsite; the exception is ICPP, where Grid III station data were used. Grid III is located approximately $1.1 \mathrm{mi}(1.8 \mathrm{~km})$ north-northwest of ICPP. Table III-4 summarizes meteorological tower locations and heights of wind measurements. The sector-averaged option was chosen for the atmospheric dispersion calculations since this reflects annual average conditions within a sector.

The majority of the input data used in this analysis were default data from CAP-88. Input parameters differing from the CAP-88 defaults are listed in Appendix D with the associated value and reference.

Where appropriate, daughter progeny were included explicitly in the source term for the releases. Examples of parent/progeny pairs incorporated in the analyses are Cs-137/Ba-137m, Sr-90/Y-90, Ru-106/Rh-106, Sb-125/Te$125 \mathrm{~m}$, and $\mathrm{Kr}-88 / \mathrm{Rb}-88$. The first three progeny of the U-238 decay series (Th-234, Pa-234, and Pa-234m) were modeled as being in secular equilibrium with U-238.

\section{Point Sources}

Emission points were modeled as either stack or ground-level releases based on EPA guidance (EPA 1989) and NCRP guidance (NCRP 1989). This guidance states that if the release height is less than or equal to 2.5 times the building height from which the stack emerges, then building downwash will lower the release height. For conservatism, these releases are included as ground-level point emissions. Stack-specific data for the INEEL emission points that were modeled as stack releases are provided in Table III-5.

\section{Diffuse Sources}

Diffuse sources at the INEEL include contaminated soil areas, fuel storage pools, evaporation ponds, etc. For this report, soil areas that were radiologically controlled (i.e., posted as a SOIL CONTAMINATION AREA) were considered potential source terms for diffuse emissions. The source term data for soils included 
conservative estimates of the surface area of each source and an estimate of the activity concentrations of specific radionuclides per unit mass or area. These data were used to estimate an annual release rate for each radionuclide, with units of $\mathrm{Ci} / \mathrm{yr}$, for input to CAP-88. Details of the source term calculations and the basis for the resuspension rate of $1 \mathrm{E}-12 \mathrm{~s}^{-1}$ for undisturbed soils are provided in Engineering Design File NES-94-002.1 maintained at the INEEL. Again, CAP 88 was used to calculate the resulting EDE. Diffuse sources were modeled as ground-level releases. Table II-10 (Section II) identified each release point for diffuse emissions, area of source, concentration, radionuclide inventory, and the release $(\mathrm{Ci} / \mathrm{g})$.

Table HI-4. Sources of Wind Data for 1997 CAP-88 Atmospheric Dispersion Modeling of Releases From The INEEL Facilities

\begin{tabular}{llll}
\hline Facility & Met Tower & Measurement & Averaging \\
& Location & Level $(\mathrm{m})$ & Period \\
\hline ANL-W & ANL & 10 & 1997 \\
& & 80 & \\
CFA & CFA & 10 & 1997 \\
ICPP & Grid II & 10 & 1997 \\
& & 61 & \\
NRF & NRF & 10 & 1997 \\
PBF & PBF & 10 & 1997 \\
RWMC & CFA & 10 & 1997 \\
TAN & TAN & 10 & 1997 \\
& & 45 & 1997 \\
\hline
\end{tabular}

a. Meteorological data from RWMC is considered unreliable because of construction activities; CFA data are substituted. 
Table III-5. INEEL Stack Data For Releases Modeled As Stack Releases

\begin{tabular}{lcccccc} 
Release Point & $\begin{array}{c}\text { Measurement } \\
\text { Level } \\
(\mathrm{m})\end{array}$ & $\begin{array}{c}\text { Stack } \\
\text { Height } \\
(\mathrm{m})\end{array}$ & $\begin{array}{c}\text { Stack } \\
\text { Diameter } \\
(\mathrm{m})\end{array}$ & $\begin{array}{c}\text { Stack } \\
\text { Radius } \\
(\mathrm{m})\end{array}$ & $\begin{array}{c}\text { Stack } \\
\text { Flow } \\
\left(\mathrm{m}^{3} / \mathrm{s}\right)\end{array}$ & $\begin{array}{c}\text { Stack } \\
\text { Velocity } \\
(\mathrm{m} / \mathrm{s})\end{array}$ \\
\hline ANL-764-001 & 80 & 61 & 1.55 & 0.78 & 23.1 & 12.2 \\
CPP-767-001 & 61 & 48.8 & 1.65 & 0.82 & 11.4 & 5.3 \\
CPP-708-001 & 61 & 76.2 & 1.98 & 0.99 & 45.9 & 14.9 \\
TAN-734-001 & 45 & 48.8 & 1.14 & 0.57 & 4.3 & 4.3 \\
TRA-710-001 & 10 & 76.2 & 1.52 & 0.76 & 5.7 & 3.2 \\
TRA-753-001 & 10 & 76.2 & 1.52 & 0.76 & 5.7 & 3.2 \\
TRA-770-001 & 10 & 76.2 & 1.52 & 0.76 & 20.0 & 11.3 \\
\hline
\end{tabular}




\section{Compliance Assessment}

\section{MEI Determination}

The MEI was calculated based on a single receptor point. The MEI location was determined by executing a series of batch files prepared for the CAP-88 computer code, one for each major release point to each of the 63 potential MEI locations. Figures 2 through 10 illustrate the sector maps from each INEEL area and the 63 potential MEI locations. The output from these batch runs were then electronically transferred onto a spreadsheet and the offsite dose was calculated at each of the 63 potential MEI locations (NES-96-004). This spreadsheet is provided as Table III-6. The INEEL MEI was then readily obtained by selecting the offsite residence with the highest EDE. Once the MEI was located, emissions from all point and diffuse sources were modeled to this INEEL MEI point. For CY 1997, the MEI was receptor \#1 (Frenchmen's Cabin). 


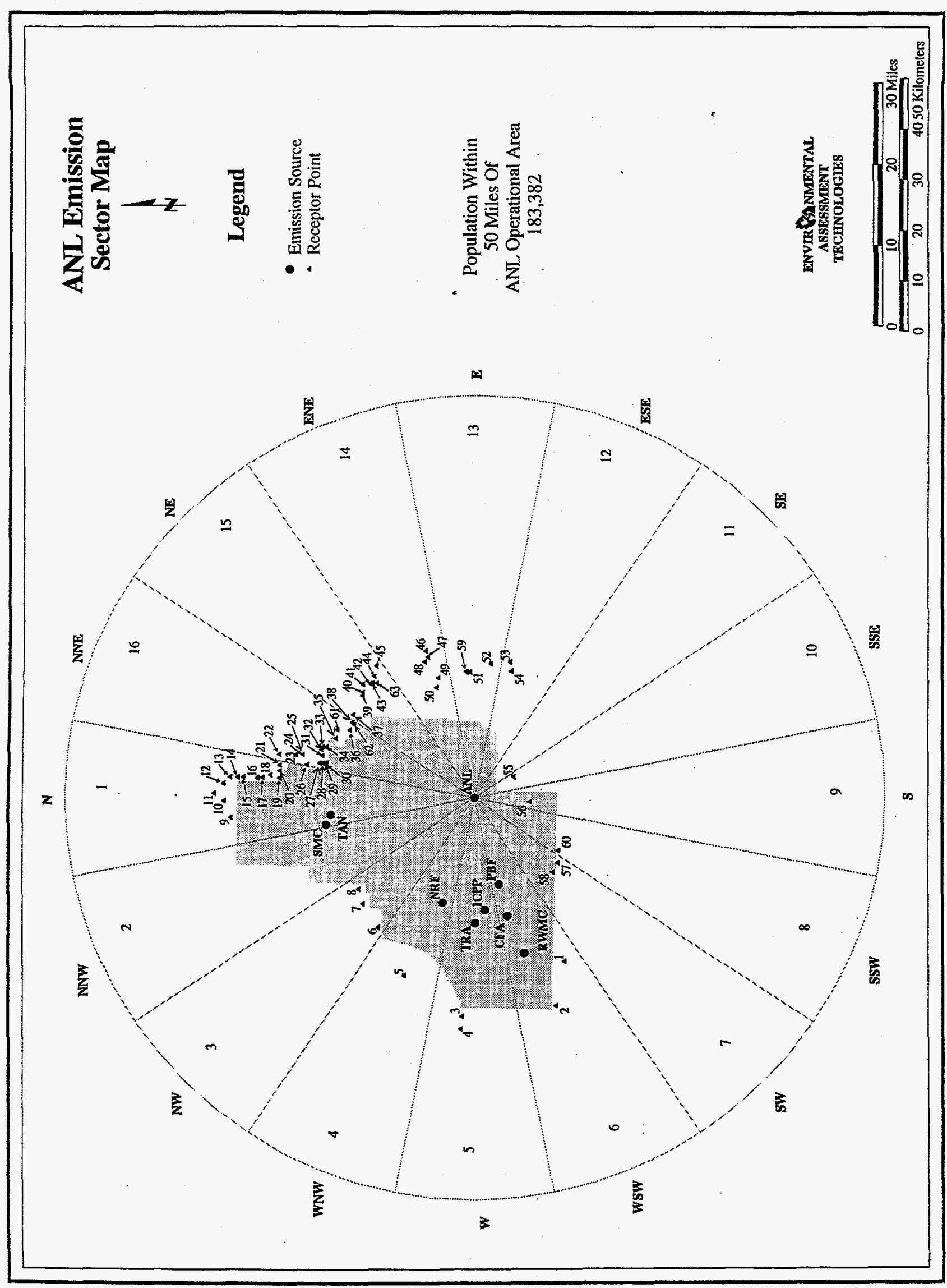

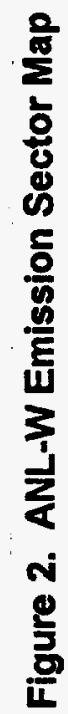


$\varepsilon$

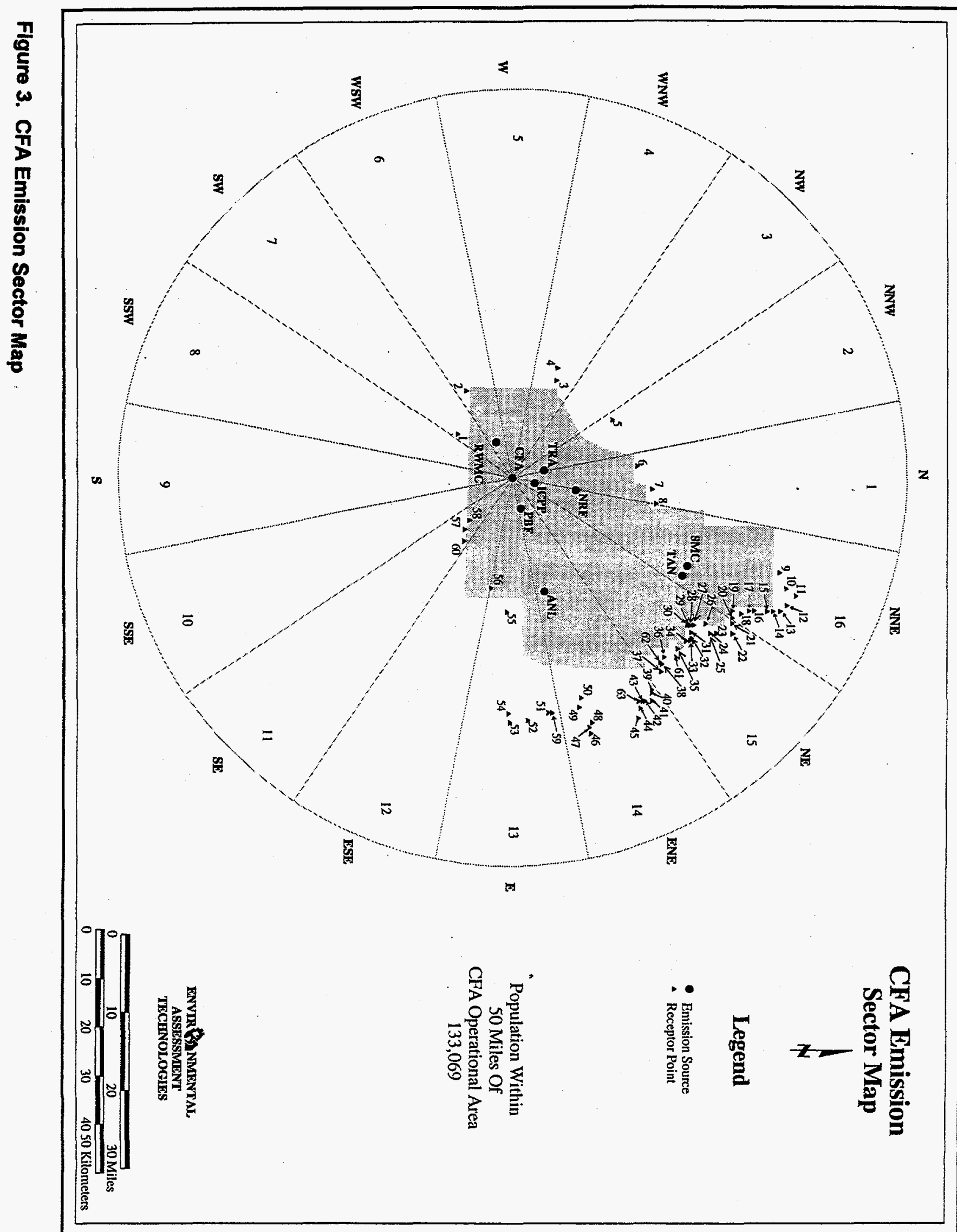




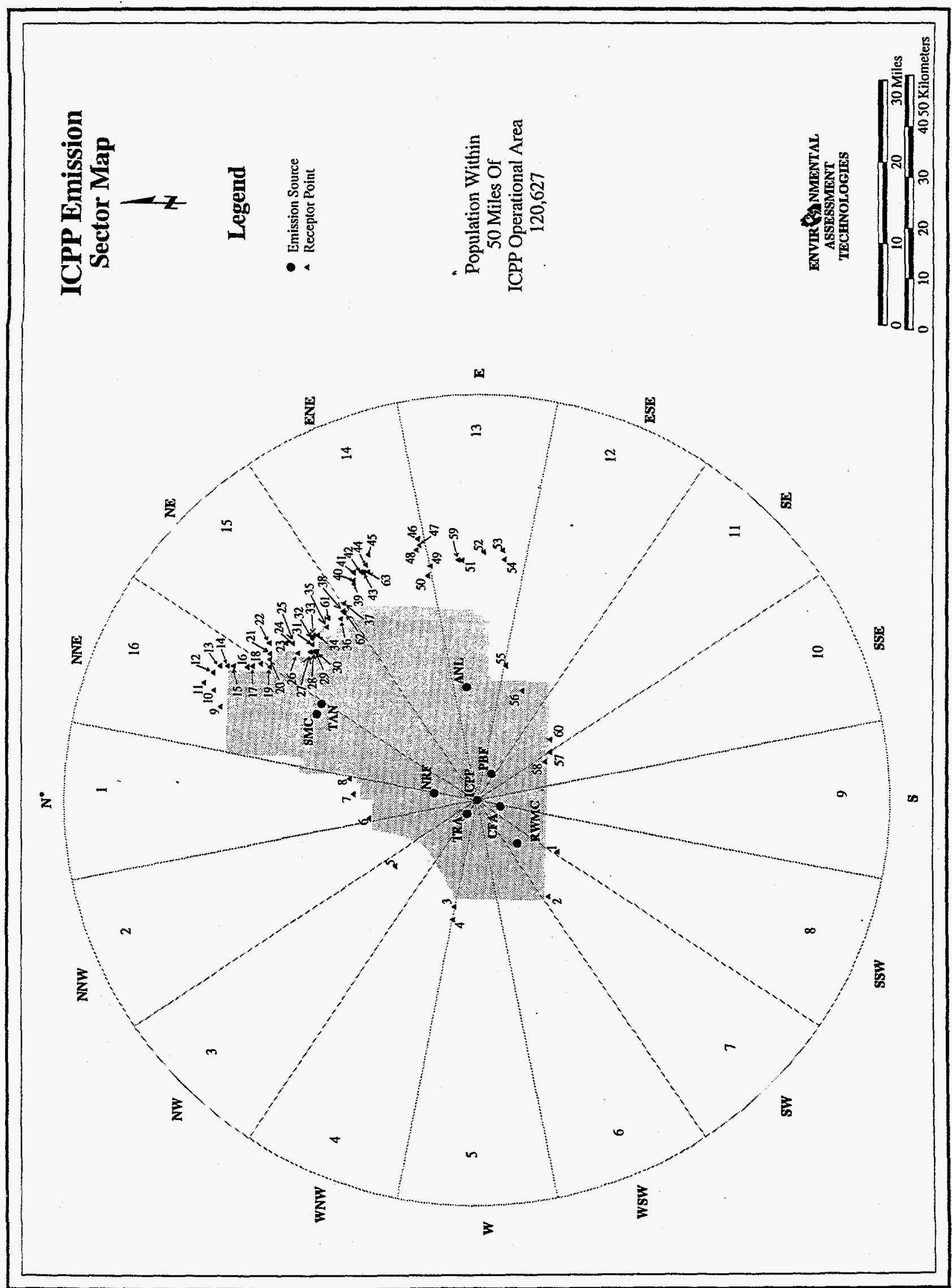

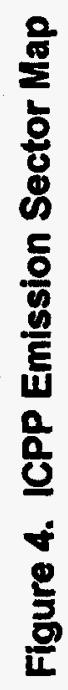




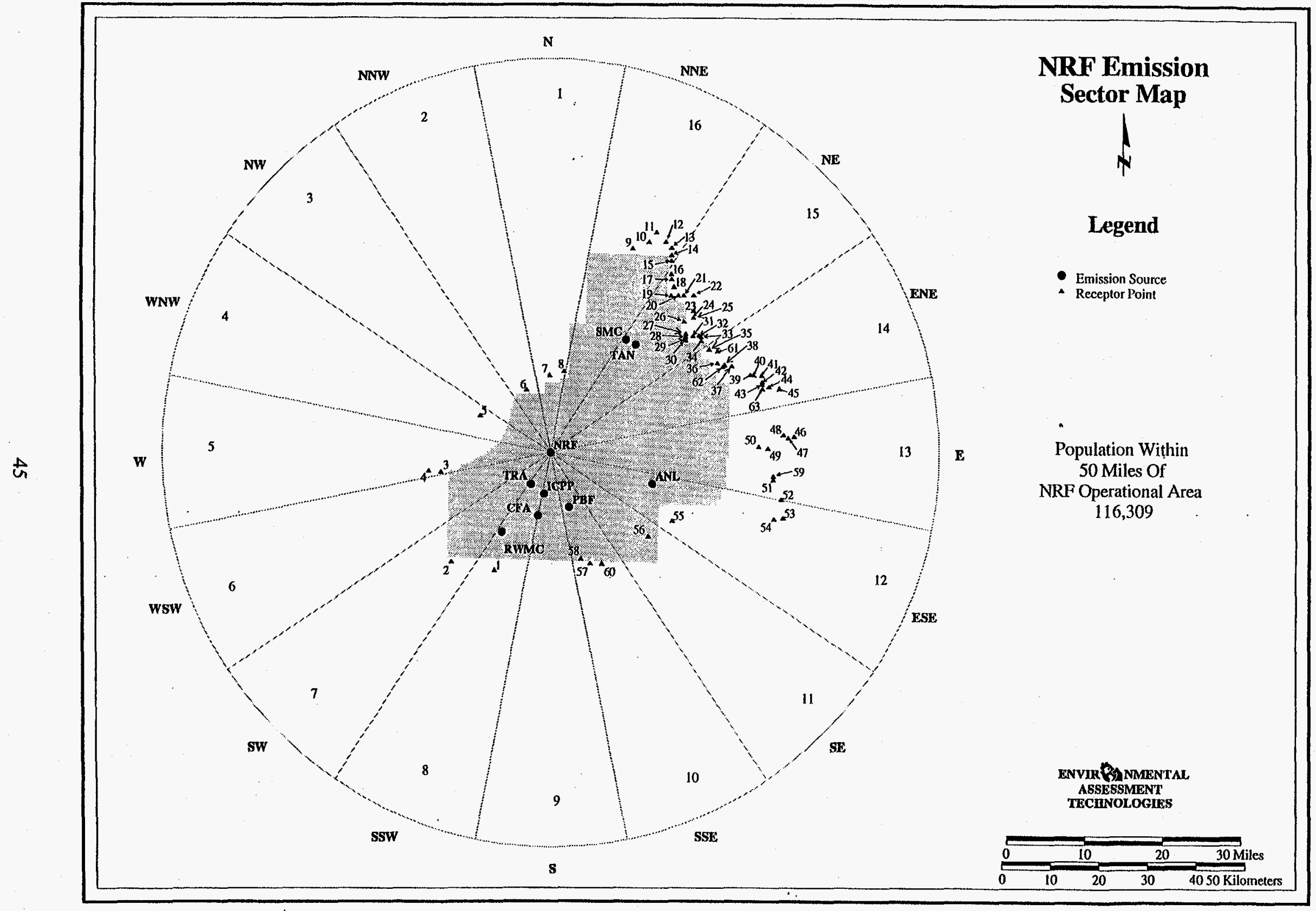

Figure 5. NRF Emission Sector Map 


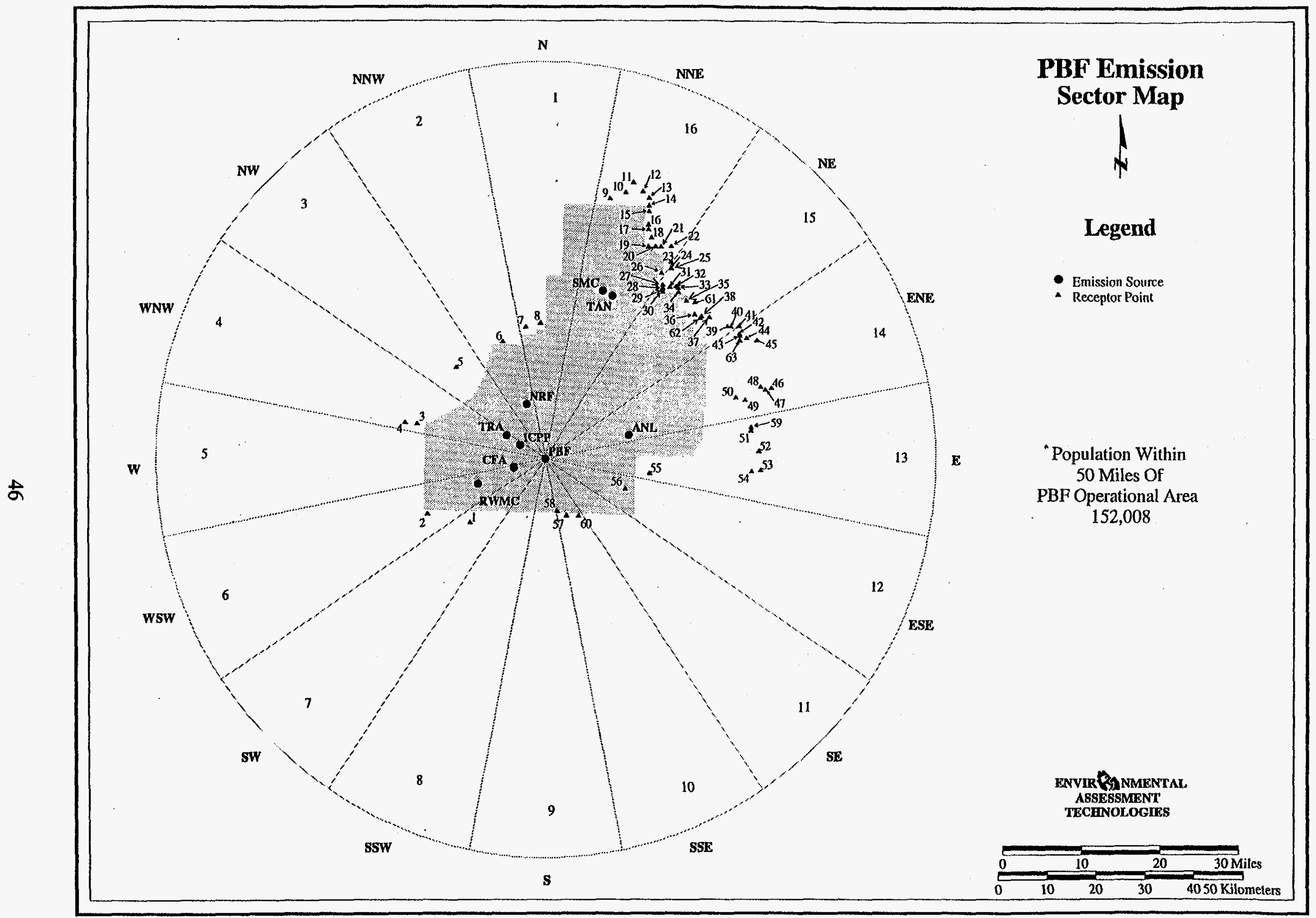

Figure 6. PBF Emission Sector Map 


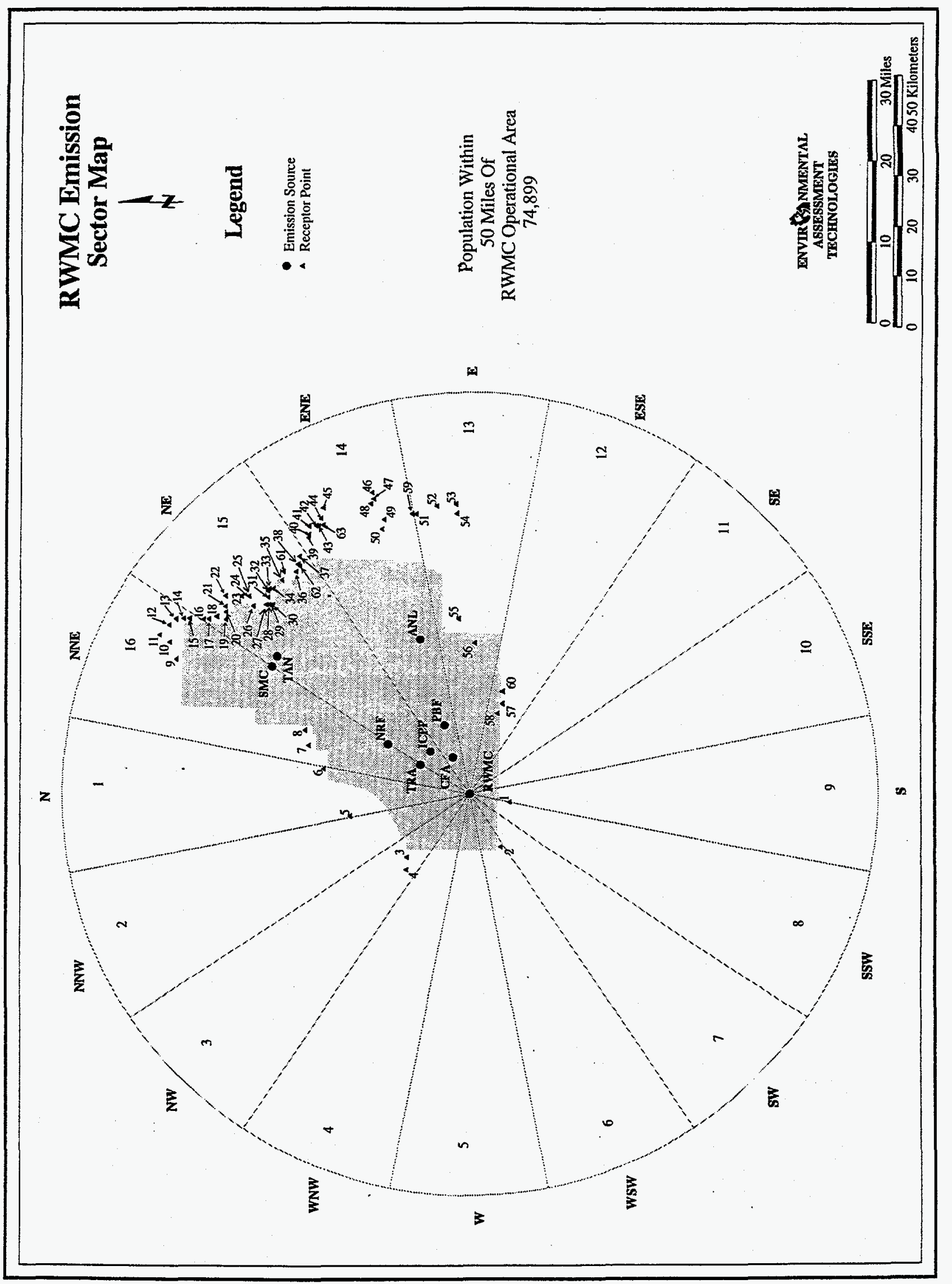

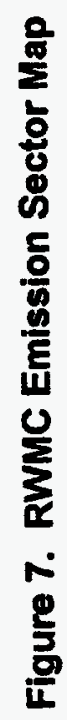




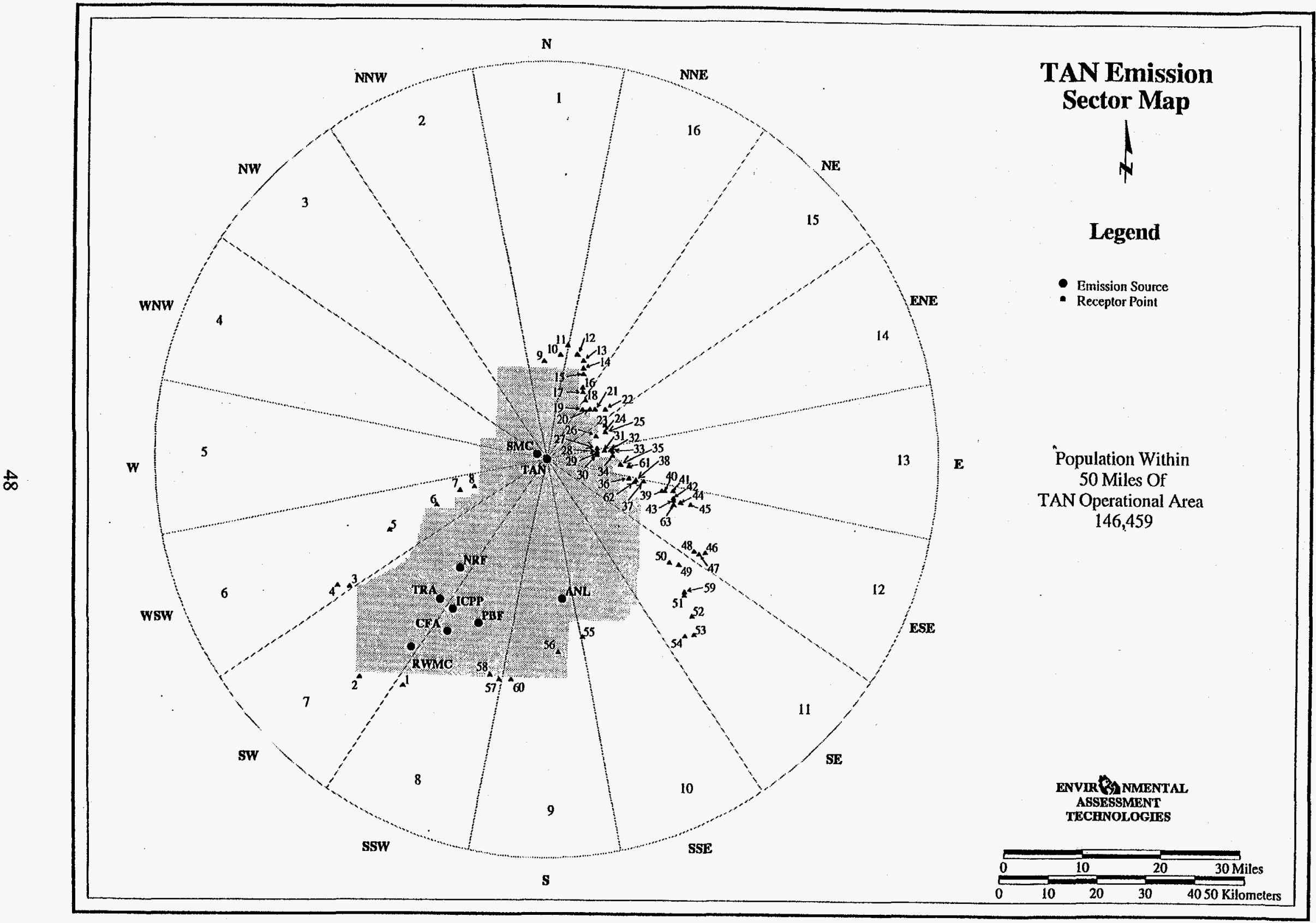

Figure 8. TAN Emission Sector Map 


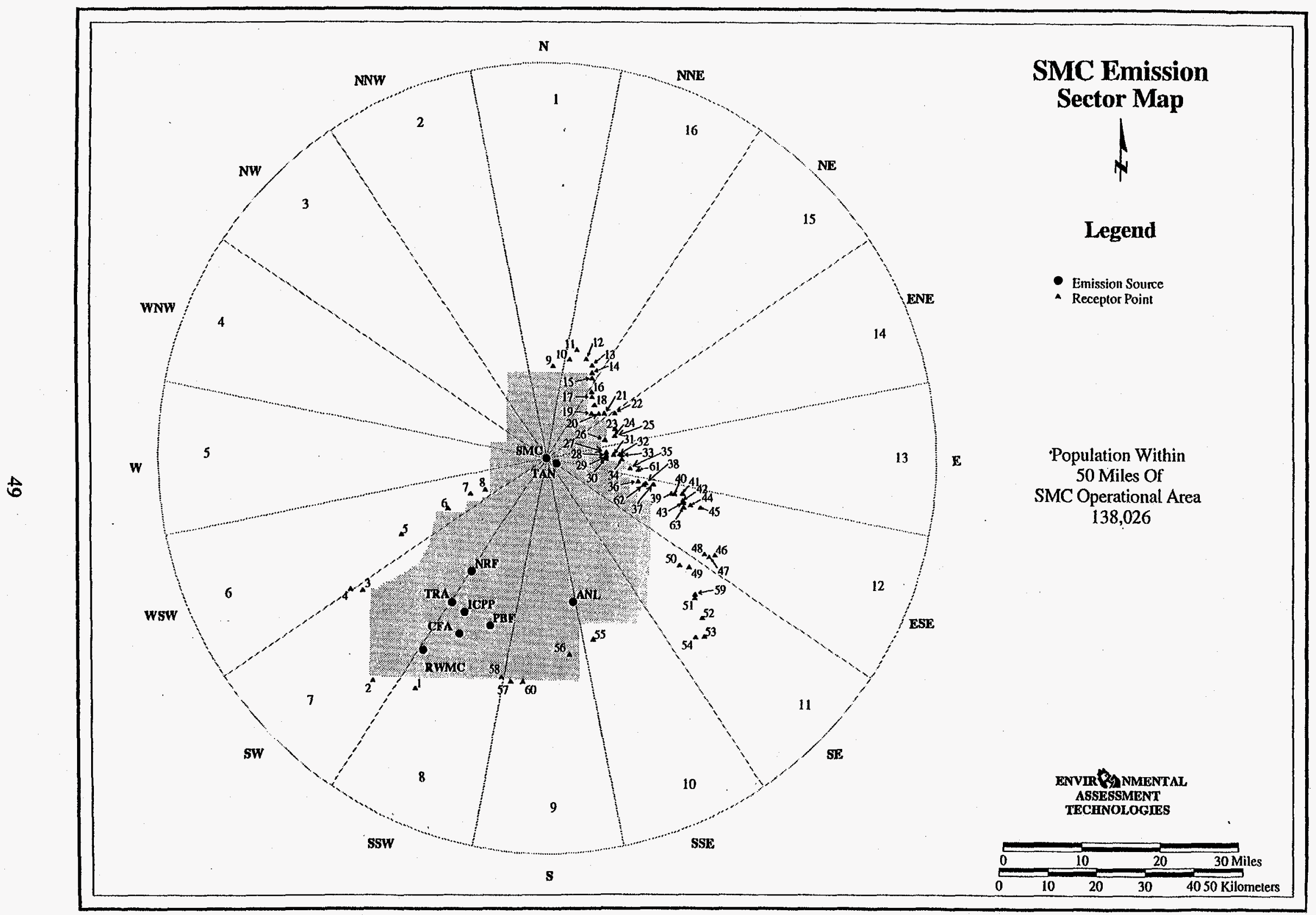

Figure 9. SMC Emission Sector Map 


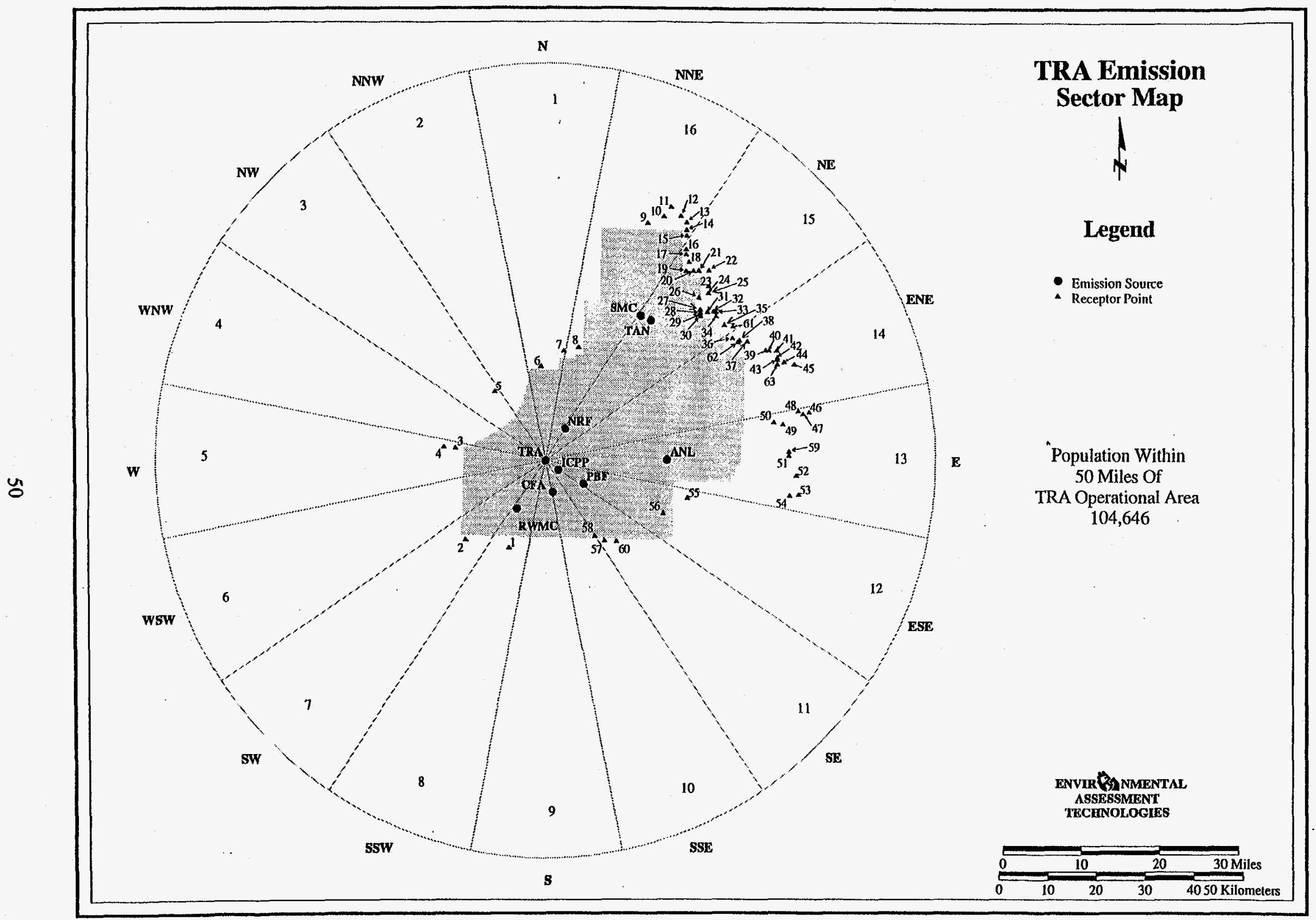

Figure 10. TRA Emission Sector Map 
Table III-6. MEl Determination Table

\begin{tabular}{|c|c|c|c|c|c|c|}
\hline \multicolumn{4}{|c|}{1997 NESHAP DOSE TO 63 RECEPTORS } & \multirow[b]{2}{*}{ RWMC } & \multirow[b]{2}{*}{ TRA-ATR } & \multirow[b]{2}{*}{ SUM } \\
\hline RECEPTOR & ANL & $\begin{array}{c}\text { ICPP } \\
\text { Gaseous }\end{array}$ & NRF & & & \\
\hline 1 & $3.24 \mathrm{E}-05$ & $1.93 \mathrm{E}-02$ & 4.31E-04 & 3.43E-03 & $3.00 \mathrm{E}-03$ & $2.62 \mathrm{E}-02$ \\
\hline 2 & $2.65 \mathrm{E}-05$ & $1.97 \mathrm{E}-02$ & $3.85 \mathrm{E}-04$ & $5.62 \mathrm{E}-04$ & $2.49 \mathrm{E}-03$ & $2.32 \mathrm{E}-02$ \\
\hline 3 & $1.17 \mathrm{E}_{-} 05$ & 2.07E-03 & $2.24 E-04$ & $3.41 \mathrm{E}-04$ & $4.26 \mathrm{E}-04$ & 3.07E-03 \\
\hline 4 & $1.10 \mathrm{E}-05$ & 2.15E-03 & $1.99 \mathrm{E}-04$ & $3.02 \mathrm{E}-04$ & $3.37 \mathrm{E}-04$ & $3.00 \mathrm{E}-03$ \\
\hline 5 & $1.06 \mathrm{E}_{-} 05$ & $2.16 \mathrm{E}-03$ & $2.56 \mathrm{E}-04$ & 4.67E-04 & $3.60 \mathrm{E}-04$ & $3.25 E-03$ \\
\hline 6 & $1.20 \mathrm{E}-05$ & $6.08 \mathrm{E}-03$ & 4.44E-04 & 3.73E-04 & $1.21 \mathrm{E}-03$ & $8.12 \mathrm{E}-03$ \\
\hline 7 & $1.27 E_{-}-05$ & $4.72 E-03$ & $5.58 \mathrm{E}-04$ & $3.68 \mathrm{E}-04$ & $9.09 \mathrm{E}-04$ & $6.57 \mathrm{E}-03$ \\
\hline 8 & $1.33 \mathrm{E}-05$ & $4.30 \mathrm{E}-03$ & 5.19E-04 & 3.47E-04 & $1.61 \mathrm{E}-03$ & $6.79 \mathrm{E}-03$ \\
\hline 9 & $1.92 E-05$ & $1.63 \mathrm{E}-03$ & $1.86 \mathrm{E}-04$ & $1.74 \mathrm{E}-04$ & $3.12 \mathrm{E}-04$ & $2.32 \mathrm{E}-03$ \\
\hline 10 & $1.87 \mathrm{E}-05$ & $1.43 \mathrm{E}-03$ & $1.74 \mathrm{E}-04$ & $1.66 \mathrm{E}-04$ & $2.77 \mathrm{E}-04$ & $2.07 \mathrm{E}-03$ \\
\hline 11 & $1.78 \mathrm{E}-05$ & $1.26 \mathrm{E}-03$ & $1.64 \mathrm{E}-04$ & $1.60 \mathrm{E}-04$ & $2.50 \mathrm{E}-04$ & $1.85 \mathrm{E}-03$ \\
\hline 12 & $1.86 \mathrm{E}-05$ & $1,32 \mathrm{E}-03$ & $1.67 \mathrm{E}-04$ & $1.61 E-04$ & $2.58 \mathrm{E}-04$ & $1.92 \mathrm{E}-03$ \\
\hline 13 & $1.92 \mathrm{E}-05$ & $1.36 \mathrm{E}-03$ & $1,70 \mathrm{E}-04$ & $1.63 \mathrm{E}-04$ & $2.63 \mathrm{E}-04$ & $1.98 \mathrm{E}-03$ \\
\hline 14 & 1.99E-05 & $1.47 \mathrm{E}-03$ & $1.75 \mathrm{E}-04$ & $1.66 \mathrm{E}-04$ & $2.79 \mathrm{E}-04$ & $2.11 \mathrm{E}-03$ \\
\hline 15 & $2.05 \mathrm{E}-05$ & $1,55 \mathrm{E}-03$ & $1.79 \mathrm{E}-04$ & $1.69 \mathrm{E}-04$ & $2.9 \mathrm{E}-04$ & $2.21 \mathrm{E}-03$ \\
\hline 16 & $220 \mathrm{E}-05$ & $176 \mathrm{E}-03$ & $1.93 \mathrm{E}-04$ & $1.85 \mathrm{E}-04$ & $6.70 \mathrm{E}-04$ & $2.83 \mathrm{E}-03$ \\
\hline 17 & $226 \mathrm{E}-05$ & 1.84E-03 & $1.98 \mathrm{E}-04$ & $1.88 \mathrm{E}-04$ & $6.95 \mathrm{E}-04$ & $2.94 \mathrm{E}-03$ \\
\hline 18 & $236 \mathrm{E}-05$ & 1.94E -03 & $2.03 \mathrm{E}-04$ & $1.92 \mathrm{E}-04$ & $7.25 \mathrm{E}-04$ & $3.08 \mathrm{E}-03$ \\
\hline 19 & $2.50 \mathrm{E}-05$ & $2.13 \mathrm{E}-03$ & $2.14 E-04$ & $1.99 \mathrm{E}-04$ & $7.88 \mathrm{E}-04$ & $3.36 \mathrm{E}-03$ \\
\hline 20 & $2.48 \mathrm{E}-05$ & $4.22 \mathrm{E}-03$ & $2.08 E_{-04}$ & $1.96 \mathrm{E}-04$ & 7.54E-04 & $5.40 \mathrm{E}-03$ \\
\hline 21 & $2.47 \mathrm{E}-05$ & $4.09 \mathrm{E}-03$ & $2.04 \mathrm{E}-04$ & $1.93 \mathrm{E}-04$ & $7.30 \mathrm{E}-04$ & $5.24 \mathrm{E}-03$ \\
\hline 22 & $3.96 \mathrm{E}-05$ & $3.84 \mathrm{E}-03$ & $1.96 \mathrm{E}-04$ & $1.88 \mathrm{E}-04$ & $6.86 \mathrm{E}-04$ & 4.95E-03 \\
\hline 23 & $4.38 \mathrm{E}-05$ & $4.34 \mathrm{E}-03$ & $2.09 \mathrm{E}-04$ & $1.97 E-04$ & $7.64 \mathrm{E}-04$ & $5.55 \mathrm{E}-03$ \\
\hline 24 & $4.59 \mathrm{E}-05$ & $4.57 \varepsilon_{-03}$ & $2.15 \mathrm{E}-04$ & $2.01 E-04$ & $8.00 E-04$ & $5.83 E-03$ \\
\hline 25 & $4.59 \mathrm{E}-0$ & $4.57 \mathrm{E}-03$ & $215 \mathrm{E}-04$ & $2.01 E-04$ & $8.00 \mathrm{E}-04$ & $5.83 \mathrm{E}-03$ \\
\hline 26 & $4.80 \mathrm{E}-05$ & $5.02 E-03$ & $229 \mathrm{E}-04$ & $2.10 \mathrm{E}-04$ & $8.79 \mathrm{E}-04$ & $6.39 \mathrm{E}-03$ \\
\hline 27 & $5.24 \mathrm{E}-05$ & $5.44 \mathrm{E}_{-}-03$ & $2.40 \mathrm{E}-04$ & $2.17 \mathrm{E}-04$ & $9.45 \mathrm{E}_{0} 04$ & $6.89 \mathrm{E}-03$ \\
\hline 28 & $5.36 \mathrm{E}-05$ & $5.57 \mathrm{E}-03$ & $2.43 \mathrm{E}-04$ & $2.19 \mathrm{E}-04$ & $9.65 \mathrm{E}-04$ & $7.05 \mathrm{E}-03$ \\
\hline 29 & $5,44 \mathrm{E}-05$ & $5.65 \mathrm{E}-03$ & $2.45 \mathrm{E}-04$ & $2.21 \mathrm{E}-04$ & $9.78 \mathrm{E}-04$ & $7.15 \mathrm{E}-03$ \\
\hline 30 & $5.52 \mathrm{E}-05$ & $5.72 E-03$ & $2.47 \mathrm{E}-04$ & $2.22 \mathrm{E}-04$ & $9.90 \mathrm{E}-04$ & $7.23 \mathrm{E}-03$ \\
\hline 31 & $5.25 \mathrm{E}-05$ & $5.26 \mathrm{E}-03$ & $2,33 \mathrm{E}-04$ & $2.14 \mathrm{E}-04$ & $9.10 \mathrm{E}-04$ & $6,67 \mathrm{E}-03$ \\
\hline 32 & $5.16 \mathrm{E}-05$ & $5.03 \mathrm{E}-03$ & $226 \mathrm{E}-04$ & $2.10 \mathrm{E}-04$ & $8.68 \mathrm{E}-04$ & $6.39 \mathrm{E}-03$ \\
\hline 33 & $5.18 \mathrm{E}-05$ & $5.00 \mathrm{E}_{-}-03$ & $2.24 \mathrm{E}-04$ & $2,09 \mathrm{E}-04$ & $8.61 \mathrm{E}-04$ & $6.35 \mathrm{E}-03$ \\
\hline 34 & $5.34 \mathrm{E}-05$ & $5.13 \mathrm{E}-03$ & $228 \mathrm{E}-04$ & $2.11 E-04$ & $8.82 \mathrm{E}-04$ & $6.50 \mathrm{E}-03$ \\
\hline 35 & $5.57 \mathrm{E}-05$ & $5.13 \mathrm{E}-03$ & $1.38 \mathrm{E}-04$ & $2.11 \mathrm{E}-04$ & $8.74 \mathrm{E}-04$ & $6.41 E-03$ \\
\hline 36 & $5,98 \mathrm{E}-05$ & $5,22 \mathrm{E}-03$ & $1.37 \mathrm{E}-04$ & $2.13 \mathrm{E}-04$ & $4.69 \mathrm{E}-04$ & $6,10 \mathrm{E}_{-}-03$ \\
\hline 37 & $7.03 \mathrm{E}-05$ & $1.84 \mathrm{E}-03$ & $1.28 \mathrm{E}-04$ & $2.04 \mathrm{E}-04$ & $4.21 \mathrm{E}-04$ & $2.66 \mathrm{E}-03$ \\
\hline 38 & $5,89 \mathrm{E}-05$ & $4.98 \mathrm{E}-03$ & $1,33 \mathrm{E}-04$ & $2.09 \mathrm{E}-04$ & $4.47 \mathrm{E}-04$ & $5,83 \mathrm{E}-03$ \\
\hline 39 & $6.77 \mathrm{E}-05$ & $1.64 \mathrm{E}-03$ & $1.19 \mathrm{E}-04$ & $2.15 \mathrm{E}-04$ & $3.76 \mathrm{E}-04$ & $2.42 \mathrm{E}-03$ \\
\hline 40 & $6.65 \mathrm{E}-05$ & $1.59 \mathrm{E}-03$ & 1.17E-04 & $2.12 \mathrm{E}-04$ & $3.67 \mathrm{E}-04$ & $2.35 \mathrm{E}-03$ \\
\hline 41 & $6.36 \mathrm{E}-05$ & $1.47 \mathrm{E}-03$ & $1.13 \mathrm{E}-04$ & $2.07 \mathrm{E}-04$ & $3.43 \mathrm{E}-04$ & $220 \mathrm{E}-03$ \\
\hline 42 & $6.59 \mathrm{E}-05$ & $1.52 \mathrm{E}-03$ & 1.14E-04 & $2.09 \mathrm{E}-04$ & $3.51 E-04$ & $2.26 \mathrm{E}-03$ \\
\hline 43 & $6.68 \mathrm{E}-05$ & $1.54 \mathrm{E}-03$ & $1.14 \mathrm{E}-04$ & $2.10 \mathrm{E}-04$ & $3.54 \mathrm{E}-04$ & $2.28 \mathrm{E}-03$ \\
\hline 44 & $6.46 \mathrm{E}-05$ & $1.45 \mathrm{E}-03$ & $1.11 \mathrm{E}-04$ & $2.06 \mathrm{E}-04$ & $3.36 \mathrm{E}-04$ & $2.17 \mathrm{E}-03$ \\
\hline 45 & $6.12 \mathrm{E}-05$ & $1.32 \mathrm{E}-03$ & $1.06 \mathrm{E}-04$ & $2.00 \mathrm{E}-04$ & $3.11 \mathrm{E}-04$ & $2.00 \mathrm{E}-03$ \\
\hline 46 & $4.88 \mathrm{E}-05$ & $1.33 \mathrm{E}-03$ & $7.94 \mathrm{E}-05$ & $2.03 \mathrm{E}-04$ & $9.24 \mathrm{E}-05$ & $1.75 \mathrm{E}-03$ \\
\hline 47 & $5.14 \mathrm{E}-05$ & $1.43 \mathrm{E}-03$ & $8.18 \mathrm{E}-05$ & $2.08 \mathrm{E}-04$ & $9.78 \mathrm{E}-05$ & $1.87 \mathrm{E}-03$ \\
\hline 48 & $5.30 \mathrm{E}-05$ & $1,49 \mathrm{E}-03$ & $8.36 \mathrm{E}-05$ & $2.11 \mathrm{E}-04$ & $1.02 E-04$ & $1.94 \mathrm{E}-03$ \\
\hline 49 & $6.29 \mathrm{E}-05$ & $6.44 \mathrm{E}-04$ & $9.05 \mathrm{E}-05$ & $2.28 \mathrm{E}-04$ & $1.20 \mathrm{E}-04$ & $1.15 \mathrm{E}-03$ \\
\hline 50 & $6.85 \mathrm{E}-05$ & $1,98 \mathrm{E}-03$ & $9.51 E-05$ & $2,36 \mathrm{E}-04$ & $1.31 E-04$ & $2.51 \mathrm{E}-03$ \\
\hline 51 & $3.41 \mathrm{E}-05$ & $6.31 \mathrm{E}-04$ & $8.73 \mathrm{E}-05$ & $1.85 \mathrm{E}-04$ & $1.16 \mathrm{E}-04$ & $1.05 \mathrm{E}-03$ \\
\hline 52 & $3.17 E-05$ & $5.79 \mathrm{E}-04$ & $8.28 E-05$ & $1.81 \mathrm{E}-04$ & $1.08 \mathrm{E}-04$ & $9.83 \mathrm{E}-04$ \\
\hline 53 & $3.02 \mathrm{E}-05$ & $5,58 \mathrm{E}-04$ & $7.13 \mathrm{E}-05$ & $1.81 \mathrm{E}-04$ & $1.04 \mathrm{E}-04$ & $945 \mathrm{E}-04$ \\
\hline 54 & $3.23 \mathrm{E}-05$ & $6.20 \mathrm{E}-04$ & $7.42 \mathrm{E}-05$ & $1.88 \mathrm{E}-04$ & $1.13 \mathrm{E}-04$ & $1.03 \mathrm{E}-03$ \\
\hline 55 & $1.64 \mathrm{E}-04$ & $1.22 \mathrm{E}-03$ & $1.32 E-04$ & $3.21 \mathrm{E}-04$ & $2.23 \mathrm{E}-04$ & 2.06E-03 \\
\hline 56 & $1.52 E-04$ & $1.64 \mathrm{E}-03$ & $1.61 E-04$ & $3.83 \mathrm{E}-04$ & $2.88 \mathrm{E}-04$ & $2.62 \mathrm{E}-03$ \\
\hline 57 & $1.41 \mathrm{E}-04$ & $4.74 \mathrm{E}-03$ & $2.59 \mathrm{E}-04$ & $6.18 \mathrm{E}-04$ & $4.81 \mathrm{E}-04$ & $6.24 \mathrm{E}-03$ \\
\hline 58 & $1.37 \mathrm{E}-04$ & $5.66 \mathrm{E}-03$ & $2.79 \mathrm{E}-04$ & $7.06 \mathrm{E}-04$ & $5.74 \mathrm{E}-04$ & $7.36 \mathrm{E}-03$ \\
\hline 59 & $3.40 \mathrm{E}-05$ & $6,28 \mathrm{E}-04$ & $8.74 \mathrm{E}-05$ & $229 \mathrm{E}-04$ & $1.16 \mathrm{E}-04$ & $1.09 \mathrm{E}-03$ \\
\hline 60 & $1.70 \mathrm{E}-04$ & $2.66 \mathrm{E}-03$ & $2.46 \mathrm{E}-04$ & $5,42 \mathrm{E}-04$ & $4.1 \pi-04$ & $4.04 \mathrm{E}-03$ \\
\hline 61 & $5.46 \mathrm{E}-05$ & $4.84 \mathrm{E}-03$ & $1.33 \mathrm{E}-04$ & $2.06 \mathrm{E}-04$ & $8.23 \mathrm{E}-04$ & $6.06 \mathrm{E}-03$ \\
\hline 62 & $6.01 \mathrm{E}-05$ & $5.10 \mathrm{E}_{-} 03$ & $1.35 \mathrm{E}-04$ & E-04 & $4.5 \mathrm{E}-04$ & $5.96 \mathrm{E}-03$ \\
\hline 63 & $6.83 \mathrm{E}-05$ & $1.57 \mathrm{E}=03$ & $115 E_{-04}$ & $2.12 \mathrm{E}-04$ & $3.59 \mathrm{E}-04$ & $2,32 \mathrm{E}_{-} 03$ \\
\hline
\end{tabular}




\section{Operational Area Modeling}

\section{Argonne National Laboratory - West}

One emission point was modeled as a stack. Table III- 5 contains the stack data used in the analyses.

- ANL-764-001 - Experimental Breeder Reactor II (EBR-II) and Fuel Conditioning Facility (FCF)

ANL-785-018 - Hot Fuel Examination Facility (HFEF) was modeled separately as a ground-level release. All remaining sources were modeled as a composite ground-level release.

The location of the INEEL MEI with respect to ANL-W was a residence, 37,219 meters (23.1 miles) westsouthwest of ANL-W.

\section{Central Facilities Area}

The RESL laboratory vents were modeled as a composite ground-level release. The location of the INEEL MEI with respect to CFA was a residence, 14,359 meters (8.9 miles) southwest of CFA.

\section{Idaho Chemical Processing Plant}

Two emission points were modeled as stacks. Table III-5 contains the stack data used in the analyses.

- ICPP FAST stack (CPP-767-001)

- ICPP Main stack (CPP-708-001).

All remaining sources were modeled as a composite ground-level release.

The location of the INEEL MEI with respect to ICPP was 18,718 meters (11.6 miles) south-southwest of ICPP.

\section{Naval Reactors Facility}

See Appendix A.

The location of the INEEL MEI with respect to NRF was a residence, 26,675 meters (16.6 miles) southsouthwest of NRF. 


\section{Power Burst Facility Area}

All sources were modeled as a composite ground-level release.

The location of the INEEL MEI with respect to PBF was 20,141 meters (12.5 miles) southwest of PBF.

\section{Radioactive Waste Management Complex}

All RWMC sources were modeled as a composite ground-level release.

The location of the INEEL MEI with respect to the RWMC was 7,976 meters (4.9 miles) south-southwest of RWMC.

\section{Test Area North (including Specific Manufacturing Capability)}

One emission point was modeled as a stack. Table III-5 contains the stack data used in the analyses.

\section{- TAN TSF Exhaust (TAN-734-001).}

The location of the nearest receptor for this stack was a residence/farm $13.0 \mathrm{~km}$ north-northeast of TAN. The remaining release points at TAN were modeled as a composite ground-level release. The location of the INEEL MEI with respect to TAN was a residence, 54,612 meters (33.9 miles) south-southwest of TAN.

The 14 SMC emission points were modeled as a composite ground-level release. The location of the INEEL MEI with respect to SMC was 54,405 meters (33.8 miles) south-southwest of SMC.

\section{Test Reactor Area}

Three emission points were modeled as stacks. Table III-5 contains the stack data used in the analyses.

- MTR Stack (TRA-710-001)

- $\quad$ ETR Stack (TRA-753-001)

- $\quad$ ATR Stack (TRA-770-001). 
The Alpha Lab, TRA Hot Cell, Chemistry Laboratories and additional emission points were modeled as a composite ground-level release.

The location of the INEEL MEI with respect to TRA was 19,172 meters (11.9 miles) south-southwest of TRA. 


\section{Construction/Modification Projects}

Section 61.94(b)(8) of 40 CFR 61 requires that an annual report identify and briefly describe all construction and modifications (completed in the applicable calendar year) for which the requirement to apply for approval to construct or modify was waived under Section 61.96. NRF construction and modifications are discussed in Appendix A. For CY 1997 no projects were completed.

- A waste characterization and segregation tent was erected at the RWMC. The moveable $15 \mathrm{ft}$ by $25 \mathrm{ft}$ long HEPA filter exhausted tent was added to Table II-5.

- A repackaging booth was constructed at the Mixed Waste Storage Facility. The repackaging booth is used to repackage mixed waste, characterize mixed waste and verify waste contents.

- An existing facility was modified by the addition of a Debris Rule Treatment Facility. Decontamination of discarded equipment and material is performed there. 
V. REFERENCES

Clawson, K.L., G. E. Start, and N. R. Ricks, 1989, Climatology of the Idaho National Engineering Laboratory and Edition, DOE/ID-12118.

EPA (U.S. Environmental Protection Agency), 1990, The Clean Air Act Assessment Package - 1988 (CAP-88), A Dose and Risk Assessment Methodology for Radionuclide Emissions to Air, Volumes 1-3, prepared by D. A. Beres, SC\&A, Inc., for the U.S. Environmental Protection Agency.

EPA, 1989, Procedures Approved for Demonstrating Compliance with 40 CFR 61, Subpart I, EPA 520/1-89-001.

NES-96-004, Method For determining The Dose Caused by the INEEL Operations for the 1995 and Later NESHAPs Annual Reports, NES-96-004.1, dated May 29, 1997.

NCRP (National Council on Radiation Protection and Measurement), 1989, Screening Techniques for Determining Compliance with Environmental Standards, NCRP Commentary No. 3.

56 
APPENDIX A

Naval Reactors Facility

Radionuclide Air Emissions Report 
Naval Reactors Facility (NRF)

National Emission Standards

for Hazardous Air Pollutants

(NESHAPs) - Radionuclides

Annual Report

June 1998 


\section{U. S. Department of Energy \\ Radionuclide Air Emissions Annual Report \\ (under Subpart H of 40 CFR Part 61) \\ Calendar Year 1997}

Site Name: Idaho National Engineering and Environmental Laboratory (INEEL)

Area: $\quad$ Naval Reactors Facility (NRF)

\section{Area Information for NRF}

Operator: Westinghouse Electric Company, a division of CBS Corporation

Address: $\quad$ P. O. Box 2068

Idaho Falls, Idaho 83403-2068

Contact: $\quad$ J. W. Solomon, Manager Naval Reactors Facility

Phone: (208) 533-5526

Owner: $\quad$ Pittsburgh Naval Reactors Office, Idaho Branch Office

$\begin{array}{ll}\text { Address: } & \text { P. O. Box } 2469\end{array}$

Idaho Falls, Idaho 83403-2469

Contact: $\quad$ T. M. Bradley, Manager Naval Reactors Idaho Branch Office

Phone: (208) 533-5317 


\section{FACILITY INFORMATION}

\section{Site Description}

The Naval Reactors Facility (NRF) is operated for the Department of Energy (DOE) by Westinghouse Electric Company, a division of CBS Corporation, and is located on the Idaho National Engineering and Environmental Laboratory (INEEL) Site (Figure 1). NRF is located approximately 8.1 miles (13,100 meters) north of the Central Facilities Area (CFA) and 6.7 miles (10,800 meters) from the nearest NEEL border. The nearest population center is Howe which is located approximately 10.1 miles (16,200 meters) from NRF. Howe has approximately 20 residents. In addition, there are individual homes, farms, and ranches located in close proximity to the INEEL boundaries surrounding NRF. Section III provides specific information concerning the distances to locations used for dose modeling.

The climate of the INEEL is characterized as semi-arid. The INEEL is located on the Snake River Plain with an elevation of approximately 5000 feet ( 1500 meters), and it is surrounded by mountains. Air masses entering the Snake River Plain from the west lose most of their moisture to precipitation prior to encountering the INEEL; therefore, annual precipitation at the INEEL is light. Winds are channeled over the Snake River Plain by bordering mountain ranges so that a southwest wind predominates over the INEEL. The second most frequent winds are from the northeast. The average air temperature, average wind speed, and the average precipitation are included in the CAP-88 computer code calculations.

The Expended Core Facility (ECF) and three naval nuclear prototypes (S1W, AlW, and S5G) are located on the developed portion of NRF, which covers 84 acres (34 hectares). ECF is a large laboratory designed to receive, handle, examine, measure, and test naval nuclear reactor fuel modules and engineering test specimens. The three prototypes, when operating, were utilized to test advanced Naval Reactors components and to train United States Navy personnel for service aboard nuclear-powered ships. The S1W, A1W, and S5G prototypes concluded operation in October 1989, January 1994, and May 1995, respectively. At present, the S1W prototype is defueled and in systems layup, the S5G prototype is defueled and is being placed into systems layup, and the AIW prototype is partially defueled and is being placed into systems layup.

\section{Source Descriptions}

NRF receives spent fuel and radioactive components from the U. S. Naval Nuclear Propulsion Program, shipped in DOEN Nuclear Regulatory Commission (NRC) approved shipping containers in accordance with Department of 


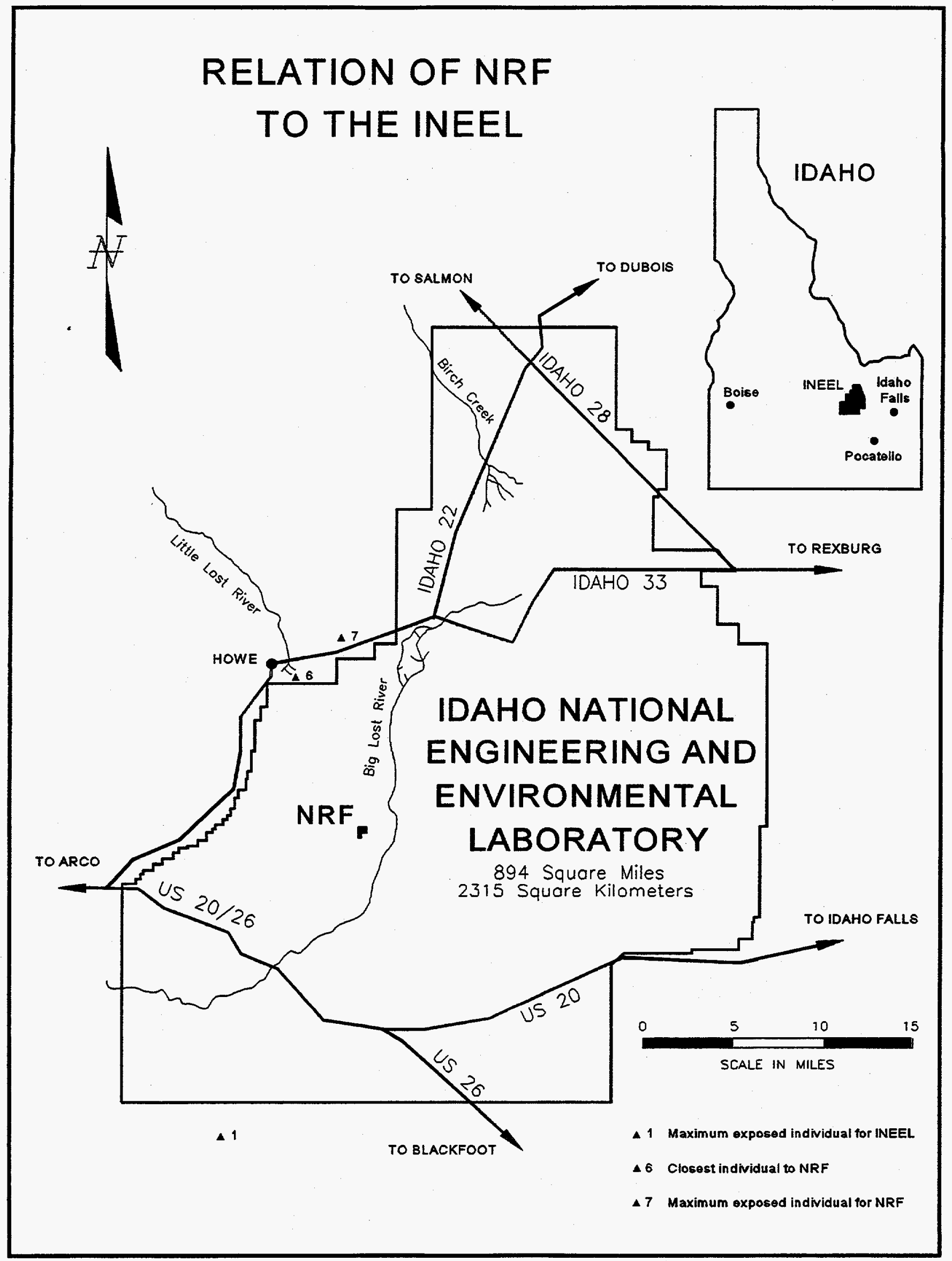

Figure 1. Relation of NRF to the INEEL. 
Transportation requirements. The shipments are processed and examined at the Expended Core Facility. After processing and examination, the naval fuel is transported to the Idaho Chemical Processing Plant (ICPP).

Radioactive materials at NRF include enriched uranium fuel with associated fission products, activation products, and activated corrosion and wear products. The principal activation products of concern are carbon-14 and tritium. The corrosion and wear products are present as insoluble metal oxide particles with cobalt- 60 being the predominant radionuclide. Various sources are used for calibrating and checking equipment, verifying shielding, and performing radiography. The sources include cobalt- 60 , cesium-137, and iridium-192. Soil with low levels of cobalt-60 and cesium-137 from fallout and from past operations is also present at NRF.

Radioactive materials are handled and processed in several areas at NRF, including shielded cells, chemical and metallurgical laboratories, machine shops, and radioactive material storage containers. Physical, chemical, and metallurgical testing of small quantities of highly radioactive material specimens is performed in the ECF shielded cells. Radioactive work is performed in appropriate containment; storage and movement of radioactive materials is under strict control. Machine shops are used to perform machining operations such as turning, milling, and drilling on a variety of metal components. Though infrequently used, special laboratory facilities are available for the chemical analysis of potentially radioactive and low-level radioactive samples.

All radioactive material is controlled by a Radioactive Material Accountability system and maintained in designated storage areas. All movements of radioactive material within the facility are performed under escort of qualified radiological controls personnel and recorded in the accountability system.

Radioactive liquids are utilized in support facilities. The majority of radioactive liquids are processed through a series of filters and demineralizers for reuse. Radioactive liquids which cannot be reused are solidified for storage or disposal as radioactive waste.

Disposable materials and waste products associated with the handling of radioactive materials are controlled and tracked as radioactive wastes. These waste materials are segregated into compactible wastes, non-compactible wastes, dewatered resin/carbon media, and solidified liquids. The wastes are temporarily stored on-site in designated storage areas until sufficient quantities accumulate to comprise a shipment to a DOE low-level disposal site.

Radionuclide emissions to the atmosphere can come from six main sources at NRF. These are (1) A1W, (2) AlW Radioactive Waste Processing System (RWPS), (3) S5G, (4) S1W, (5) ECF, and (6) fugitive sources. 
(1) The shutdown A1W prototype plant (Buildings 616A - C and 617A - C) handles chromated water and plant discharge water, which is reused after radionuclides and impurities are removed. As part of the defueling and systems layup preparations, AlW also handles contaminated materials such as tools, equipment, anti-contamination clothing, and contaminated waste.

(2) The A1W RWPS (Building 628A) is used for the removal of radionuclides and impurities from primary plant water drained from the AlW prototype plant during defueling and systems layup preparations.

(3) The shutdown S5G prototype plant (Building 633A - B) handles chromated water and plant discharge water, which is reused after radionuclides and impurities are removed. A radioactive waste processing system is used for the removal of radionuclides and impurities from primary plant water from the S5G prototype plant. As part of the systems layup preparations, S5G also handles contaminated materials such as tools, equipment, anti-contamination clothing, and contaminated waste.

(4) The S1W prototype (Building 601) is no longer operational and has been inactivated; however, there are some ventilation systems which are still in use. Work is done with radioactive material during chemistry analyses in the NRF Chemistry wing of this building. Other ventilation systems in the building are in use to control the buildup of naturally occurring radon.

(5) The Expended Core Facility (ECF Building 618) handles spent fuel from naval cores and contaminated materials such as anti-contamination clothing, tools, and other equipment. Radioactive water is present in the pits where fuel is located. Analyses are performed on radioactive materials in chemistry laboratories in this building. ECF uses excess decontaminated water from the AIW and $S 5 G$ prototypes to replenish evaporation from the large water pits.

(6) Fugitive sources are diffuse emissions not associated with a specific building vent. These sources represent fugitive soil emissions and portable blowers used on temporary containments that do not exhaust directly to the environment or to an area exhausted by a permanent ventilation system. Portable blowers are used to ensure negative pressure in containments, provide particulate control, and, in some confined spaces, maintain occupational health and safety standards. Fugitive soil sources are emissions from defined areas surrounding NRF which potentially contain low levels of radioactivity that are exposed to the wind. 


\section{AIR EMISSIONS DATA}

NRF has a number of stacks and vents with the potential to emit low quantities of radionuclides. These emissions are monitored and calculated by NRF, and the results are reported to the INEEL Environmental System (IES) on a monthly basis. The data is included in the calculation of the INEEL's annual effective dose equivalent (EDE) to members of the public.

Continuous monitoring is required by 61.93 (b) of $40 \mathrm{CFR} 61$, Subpart $\mathrm{H}$, for emission points that have a potential to emit radionuclides in quantities that could result in an EDE to a member of the pubic in excess of 1 percent of the 10 millirem per year NESHAPs' standard, which is 0.1 millirem. As part of the INEEL assessment of radiological emission points, NRF evaluated all emission sources in 1990, as reported in DOE/ID-10310, NESHAPs 40 CFR 61.93 Monitoring Requirements for Radiological Emission Sources at INEL. An evaluation was again performed for the 1995 INEEL Title V, Tier I Operating Permit Application. None of the emission points at NRF qualify for the continuous monitoring requirement; all emission points are below the 0.1 millirem per year criteria. Confirmatory evaluations are performed annually to verify that emissions are below 1 percent of the standard.

Table II-1 identifies potential point sources of radionuclide air emissions. The table contains: identification codes for area, building, and vent; a general description for each of the potential emission points; a description of the effluent controls and their efficiencies, if applicable; and those emission sources which were monitored.

Table II-2 lists the combined radionuclide emissions from the point sources for calendar year 1997. This data represents those sources which are routinely monitored. Unmonitored emissions, which are calculated, are also included.

Table II-3 identifies potential fugitive sources of radionuclide air emissions. This category contains two types of sources. The first type includes buildings which do not have a stack or vent. The second type identifies areas where soil which potentially contain low levels of radioactivity is exposed to the wind. The table contains: codes for area, building or location, and an identification code for tracking; a general description for each of the potential emission points; a description of the effluent controls and their efficiencies, if applicable; and those emission sources which were monitored.

Table II-4 lists the combined radionuclide emissions from the fugitive sources for calendar year 1997. This table includes measured values for those sources which are routinely monitored and calculated values for unmonitored emissions. 
Tables II-2 and II-4 include gross alpha and gross beta radioactivity. For determining the EDE, the gross alpha is modeled as plutonium-239 and the gross beta is modeled as strontium-90 with yttrium-90 daughter progeny. This is consistent with other facilities located on the INEEL site. For fugitive soil sources, cesium-137 and the daughter progeny barium- $137 \mathrm{~m}$ are included. 
Table II-1. NRF Potential Radiological Air Emission Point Sources

\begin{tabular}{|c|c|c|c|c|c|c|}
\hline \multicolumn{4}{|c|}{ NAVAL REACTORS FACIUTY ORF } & \multicolumn{3}{|c|}{ Nearest Receptor 1379 meters NWW. } \\
\hline AREA & BLEG. & VENI & SOURCE DESCRIPTION & $\begin{array}{l}\text { EFRLUENT CONTRQO } \\
\text { OESCRIPTION }\end{array}$ & EFFICJENCY & MONITOREO? \\
\hline NRF & 601 & 019A & S1W MAIN STACK EXHAUST: CHEMISTRY LABORATORY FUME HOODS & HEPA FILTER & $99.95 \%$ & $\mathrm{x}$ \\
\hline NRF & 601 & 023 & S1W REACTOR COMPARTMENT EXHAUST & HEPA FILTER & $99.95 \%$ & $\square$ \\
\hline NRF & 601 & HBRV & S1W HIGH BAY ROOF VENTS & NONE $^{3}$ & & $\mathrm{x}$ \\
\hline NRF & 616 & 012,021 & A1W OPERATIONS BUILDING & NONE $^{3}$ & & $x$ \\
\hline NRF & 616 & 039 & A1W RADIOCHEMISTRY AND COUNTING ROOMS & HEPA FILTER & $99.95 \%$ & $\square$ \\
\hline NRF & 616 & PCMA & A1W PRIMARY COMPONENTS MAINTENANCE AREA AND EXTENSION & NONE $^{3}$ & & $\square$ \\
\hline NRF & $616 A$ & 002 & A1W REACTOR COMPARTMENT EXHAUST & HEPA FILTER & $99.95 \%$ & $\mathrm{x}$ \\
\hline NRF & 617 & 002 & A1W MACHINERY ROOM SPACES EXHAUST & NONE $^{3}$ & & $\square$ \\
\hline NRF & 617 & 013 & A1W REACTOR COMPARTMENT 3A EXHAUST & NONE & & $x$ \\
\hline NRF & 617 & 020,021 & A1W REACTOR COMPARTMENT 3B EXHAUST & NONE & & $x$ \\
\hline NRF & 618 & 099 & ECF STACK NUMBER 1 & $\begin{array}{l}\text { HEPA FILTER } \\
\text { CARBON FILTER } \\
\end{array}$ & $\begin{array}{l}99.95 \% \\
99.9 \% \\
\end{array}$ & $\otimes$ \\
\hline NRF & 618 & 103 & ECF STACK NUMBER 2 & $\begin{array}{l}\text { HEPA FILTER } \\
\text { CARBON FILTER }\end{array}$ & $\begin{array}{l}99.95 \% \\
99.9 \%\end{array}$ & $\otimes$ \\
\hline NRF & 618 & HBRV & ECF HIGH BAY ROOF VENTS & NONE & & $x$ \\
\hline NRF & $628 \mathrm{~A}$ & 006 & TANK VENT & HEPA FILTER & $99.95 \%$ & $\square$ \\
\hline NRF & 633A & 057 & S5G RADIOACTIVE AREA VENTILATION (RAV) SYSTEM & HEPA FILTER & $99.95 \%$ & $x$ \\
\hline NRF & 633A & HBRV & S5G HIGH BAY ROOF VENTS & NONE $^{3}$ & & $x$ \\
\hline NRF & MSC & - & MISCELLANEOUS POINT SOURCES ${ }^{4}$ & HEPA FILTER & 99.95\% & $\square$ \\
\hline
\end{tabular}

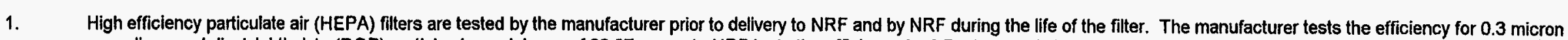
monodispersed dioctylphthalate (DOP) particles to a minimum of 99.97 percent. NRF tests the efficiency for 0.7 micron polydispersed DOP particles to a minimum of 99.95 percent.

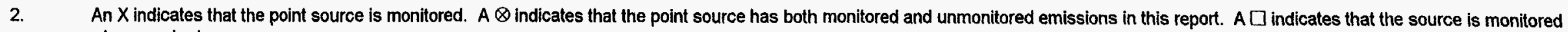
When required.

3. Subsystems which exhaust within the areas ventilated by these sources may have HEPA filters.

4. This includes sources such as temporary systems required to support defueling and systems layup work. 
Table II-2. Point Source Releases From NRF During 1997

\begin{tabular}{|c|c|c|}
\hline Radionuclide & $\begin{array}{l}\text { Release } \\
\text { (curies) }\end{array}$ & $\begin{array}{c}\text { Release } \\
\text { (becquerels)* }\end{array}$ \\
\hline Carbon-14 (C-14) & 8.1E-01 & $3.0 \mathrm{E}+10$ \\
\hline Gross alpha \{modeled as plutonium-239 & $3.8 \mathrm{E}-06$ & $1.4 \mathrm{E}+05$ \\
\hline \multicolumn{3}{|l|}{ (Pu-239)\} } \\
\hline Gross beta \{modeled as strontium-90 & $6.7 \mathrm{E}-05$ & $2.5 \mathrm{E}+06$ \\
\hline \multicolumn{3}{|l|}{$(\mathrm{Sr}-90) /$ yttrium-90 $(\mathrm{Y}-90)\}$} \\
\hline Tritium (H-3) & $7.8 \mathrm{E}-02$ & $2.9 E+09$ \\
\hline Mercury-203 (Hg-203) & $3.0 \mathrm{E}-07$ & $1.1 \mathrm{E}+04$ \\
\hline Iodine-131 (I-131) & $1.7 \mathrm{E}-05$ & $6.3 E+05$ \\
\hline Osmium-191 (Os-191) & $1.5 \mathrm{E}-05$ & $5.6 \mathrm{E}+05$ \\
\hline
\end{tabular}

* Note: 1 curie equals $3.7 \mathrm{E}+10$ becquerels. 
Table II-3. NRF Potential Radiological Air Emission Fugitive Sources

\begin{tabular}{|c|c|c|c|c|c|c|}
\hline \multicolumn{4}{|c|}{ NAVAI REACTORS FACIITY N NRF) } & \multicolumn{3}{|c|}{ Nearest Receptor / 13714 meters NVW } \\
\hline AREA & OOCATION & CODE & SOURCE DESCRIPTION & 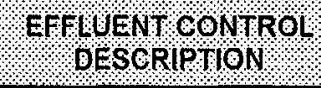 & EFFIOIENCY & MONITORED \\
\hline NRF & 605 & (NONE) & SIW PUMP HOUSE: FUGITIVE & NONE $^{3}$ & & $x$ \\
\hline NRF & 631 & (NONE) & RADIOACTIVE COMPONENT STORAGE WAREHOUSE: FUGITIVE & NONE $^{3}$ & & $x$ \\
\hline NRF & 642 & (NONE) & CELITE TANK HOUSE: FUGITIVE & NONE $^{3}$ & & $\mathrm{x}$ \\
\hline NRF & MSC & - & MISCELLANEOUS FUGITIVE SOURCES` & HEPA FILTER & $99.95 \%$ & $\square$ \\
\hline NRF & SOIL & 001 & $\begin{array}{l}\text { S1W LEACHING PIT (NOT IN USE): FUGITIVE SOIL SURROUNDING } \\
\text { COVERED AREA }\end{array}$ & NONE & & \\
\hline NRF & SOIL & 002 & $\begin{array}{l}\text { A1W LEACHING PIT (NOT IN USE) AND SURROUNDING AREA: FUGITIVE } \\
\text { SOIL }\end{array}$ & NONE & & \\
\hline NRF & SOIL & 003 & SOUTHWEST SEWAGE LAGOON: FUGITIVE SOIL IN DRY SEWAGE & NONE & & \\
\hline NRF & SOIL & 004 & NRF PERIMETER AREA: FUGITIVE SOIL & NONE & & \\
\hline
\end{tabular}

1. High efficiency particulate air (HEPA) filters are lested by the manufacturer prior to delivery to NRF and by NRF during the life of the filter. The manufacturer tests the efficiency for 0.3 micron monodispersed dioctyiphthalate (DOP) particles to a minimum of 99.97 percent. NRF tests the efficiency for 0.7 micron polydispersed DOP particles to a minimum of 99.95 percent.

2. An $\mathrm{X}$ indicates that the source is monitored. $\mathrm{A} \square$ indicates that the source is monitored when required. $\mathrm{A}$ blank indicates that the emissions are not monitored.

3. Subsystems which exhaust within the areas ventilated by these sources may have HEPA filters.

4. This includes sources such as temporary systems required to support defueling and systems layup work. 
Table II-4. Fugitive Source Releases From NRF During 1997

\begin{tabular}{|c|c|c|}
\hline Radionuclide & $\begin{array}{l}\text { Release } \\
\text { (curies) }\end{array}$ & $\begin{array}{c}\text { Release } \\
\text { (becquerels)* }^{*}\end{array}$ \\
\hline Cobalt-60 (Co-60) & $1.8 \mathrm{E}-06$ & $6.7 E+04$ \\
\hline $\begin{array}{c}\text { Cesium-137 (Cs-137)/barium-137m } \\
\text { (Ba-137m) }\end{array}$ & $3.6 \mathrm{E}-05$ & $1.3 E+06$ \\
\hline $\begin{array}{l}\text { Gross alpha }\{\text { modeled as plutonium-239 } \\
\text { (Pu-239)\} }\end{array}$ & 7.3E-08 & $2.7 \mathrm{E}+03$ \\
\hline $\begin{array}{l}\text { Gross beta }\{\text { modeled as strontium-90 } \\
\qquad(\mathrm{Sr}-90) / \text { yttrium-90(Y-90)\} }\end{array}$ & $1.2 \mathrm{E}-06$ & $4.4 \mathrm{E}+04$ \\
\hline
\end{tabular}

* Note: 1 curie equals $3.7 \mathrm{E}+10$ becquerels. 


\section{DOSE ASSESSMENTS}

\section{Summary}

Table III-1 summarizes the EDE results for point sources, fugitive sources, and all sources combined. The EDE from all NRF sources at the receptor receiving the highest dose is $5.6 \times 10^{-4}$ millirem $\left(5.6 \times 10^{-3}\right.$ microsievert $)$ and occurred at a location 15.8 kilometers north of NRF. The EDE from point sources is $5.6 \times 10^{-4}$ millirem $\left(5.6 \times 10^{-3}\right.$ microsievert) and the EDE from fugitive sources is $1.3 \times 10^{-6}$ millirem $\left(1.3 \times 10^{-5}\right.$ microsievert). The NRF EDE is for information only. The emissions for which this dose was calculated have been included in the INEEL calculation of the total EDE. It is the INEEL total EDE that is used to demonstrate compliance with the 40 CFR 61.92 standard of 10 millirem per year.

Subpart $H$ of 40 CFR 61 requires that emissions of radionuclides to the ambient air from Department of Energy facilities shall not exceed those amounts which would cause any member of the public to receive in any year an EDE of 10 millirem ( 100 microsievert) per year. "Member of the public" is any offsite point where there is a residence, school, business, or office. For compliance purposes, this EDE is calculated for all emission sources on the INEEL. The EDE calculated for NRF is for information only; the emissions used for the NRF EDE have been included in the INEEL calculation of the EDE for the maximally exposed individual.

\section{Description of Dose Model and Summary of Input Parameters}

\section{General}

The CAP-88 computer code is used to calculate the EDE from NRF releases. CAP-88 is approved for use by the Environmental Protection Agency (EPA) for demonstrating compliance with 40 CFR 61 Subpart H, "National Emission Standards for Emissions of Radionuclides Other Than Radon From Department of Energy Facilities." Two other approved computer codes are available for calculating the EDE. The COMPLY computer code was not used because the maximally exposed individual for emissions is more than 3 kilometers from emission sources. The AIRDOS-PC code was not used because it does not include several radionuclides that are released from facilities at the INEEL.

The output from CAP-88 is the EDE, which includes the 50-year committed and external EDEs. The committed EDE calculates internal exposure from ingestion and inhalation pathways, while the external EDE determines exposure from ground deposition and air immersion. The dose conversion factors are from the RADRISK dosimetric data base. 
The population data set used in the CAP- 88 calculations is based on the 1990 census. Locations were measured during the INEEL 1995 aerial survey. It is assumed that members of the population grow their own vegetables and raise their own beef and dairy cattle.

The wind file used in the CAP-88 calculations is derived from data obtained from the National Oceanic and Atmospheric Administration (NOAA). The wind data was collected during 1997 at the NRF 10-meter meteorological tower. Calm hours are apportioned into the lowest wind speed class. The sector-averaged option is used for the atmospheric dispersion calculations, because this reflects annual average conditions within a sector.

Where appropriate, daughter progeny were included in the source term for releases. Gross beta is modeled as strontium-90 with yttrium-90 daughter progeny; this is consistent with other facilities located on the INEEL site. The daughter progeny barium- $137 \mathrm{~m}$ is included for cesium- 137 .

\section{Point Sources}

Since none of the emission points at NRF are more than 2.5 times the building height, all emissions were considered to occur at ground level. All emissions from NRF were modeled as a single release point.

\section{Fugitive Sources}

Fugitive soil releases, included in fugitive sources, were calculated using soil resuspension rates of approximately one percent per year. The method used for determining resuspension rates is described in DOE/TIC-22800, Transuranic Elements in the Environment, by Wayne C. Hanson.

\section{Compliance Assessment}

\section{Maximally Exposed Individual}

Various receptors near the INEEL boundary were evaluated when calculating the highest EDE for 1997. The nearest receptor to NRF is a residence located approximately 8.5 miles (13.7 kilometers) to the north-northwest of NRF (Figure 1, Location 6), but it did not receive the highest dose. The receptor that received the highest dose from NRF is a residence located approximately 9.8 miles (15.8 kilometers) to the north of NRF (Figure 1, Location 7 ). The nearest receptor did not receive the highest dose, because it was not in the direction of the prevailing winds. The receptor that received the highest dose from all INEEL sources is located approximately 16.6 miles (26.7 kilometers) to the south-southwest of NRF (Figure 1, Location 1). The EDE results presented for NRF are for the receptor that received the highest dose from NRF (Figure 1, Location 7). 
Table III-1. Summary of 1997 Effective Dose Equivalents from Point Sources and Diffuse Sources at NRF

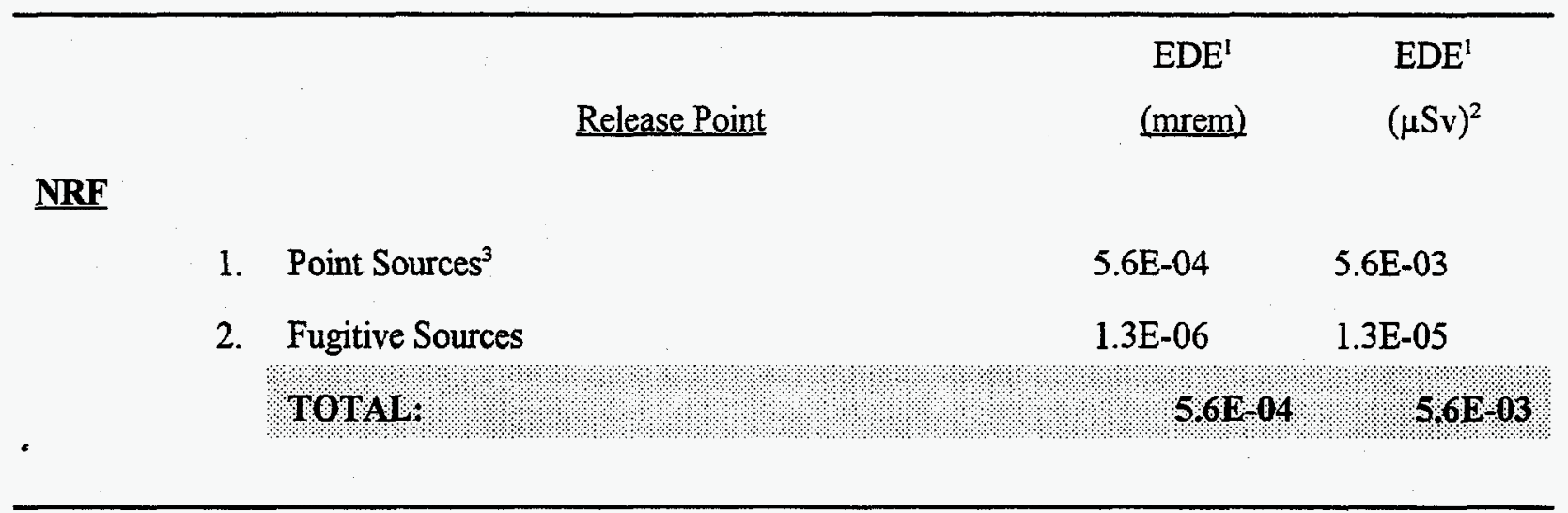

1. The EDE shown is for the NRF maximally exposed individual (Figure 1, Location 7).

2. Note: 1 millirem equals 10 microsievert.

3. This includes stack releases being modeled as ground level releases due to building wake effects. 


\section{Statement of Certification}

I certify under penalty of law that I have personally examined and am familiar with the information submitted in this report, the 1997 Naval Reactors Facility National Emission Standards for Hazardous Air Pollutants (NESHAPs) Radionuclide Annual Report. Based on my inquiry of those individuals immediately responsible for obtaining the information, I believe that the submitted information is true, accurate, and complete. I am aware that there are significant penalties for submitting false information including the possibility of fine and imprisonment. See, 18 U.S.C. 1001.

Owner Signature:

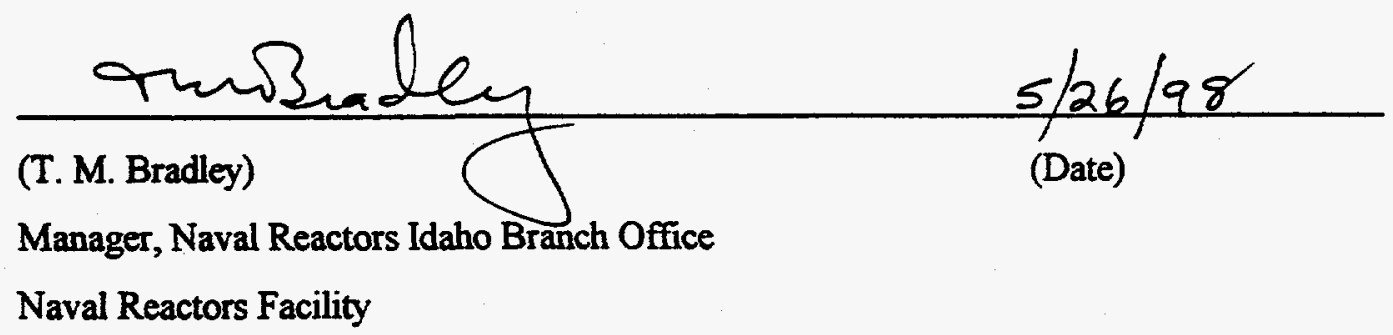




\section{Additional Information}

The EPA requires in 40 CFR 61 Subpart $H$ that a brief description of all construction and modifications which were completed in 1997, but for which the requirement to apply for approval to construct or modify was waived, be included. No new construction or modification was completed in 1997 which would result in a potential increase in radionuclide airborne emissions. 


\section{SUPPLEMENTAL INFORMATION}

The following information is provided at the request of the Department of Energy Headquarters and is not required as part of the annual National Emission Standards for Hazardous Air Pollutants reporting requirements (under 40 CFR Section 61.94).

REQUEST: Provide an estimate of the collective effective dose equivalent (person-rem per year) for 1997 releases.

An estimate of the collective effective dose equivalent (person-rem per year) will be provided in the Idaho National Engineering and Environmental Laboratory Site Environmental Report for Calendar Year 1997 $\{D O E / I D-12082(97)\}$. This collective EDE is calculated using the mesoscale diffusion (MDIFF) model and not CAP-88.

REQUEST: Provide information on the status of compliance with Subparts $Q$ and $T$ of 40 CFR Part 61 if pertinent.

Subpart Q of 40 CFR Part 61, National Emission Standards for Radon Emissions From Department of Energy Facilities, is not applicable to the Naval Reactors Facility. Subpart T of 40 CFR Part 61, National Emission Standards for Radon Emissions From the Disposal of Uranium Mill Tailings, is not applicable to the Naval Reactors Facility.

REQUEST: Provide information on radon-220 emissions from sources containing uranium-232 and thorium-232 where emissions potentially can exceed 0.1 millirem ( 1 microsievert) per year to the public or 10 percent of the non-radon dose to the public.

The Naval Reactors Facility does not have any sources of uranium-232 or thorium-232 emissions that potentially can exceed 0.1 millirem ( 1 microsievert) per year to the public or 10 percent of the non-radon dose to the public.

REQUEST: Provide information on non-disposal and non-storage sources of radon-222 emissions where emissions potentially can exceed 0.1 millirem ( 1 microsievert) per year to the public or 10 percent of the non-radon dose to the public. 
The Naval Reactors Facility does not have any non-disposal or non-storage sources of Radon-222 emissions that potentially can exceed 0.1 millirem ( 1 microsievert) per year to the public or 10 percent of the nonradon dose to the public.

REQUEST: For the purpose of assessing facility compliance with the National Emission Standards for Hazardous Air Pollutants effuent monitoring requirements of Subpart $\mathrm{H}$ under Section 61.93(b), give the number of emission points subject to the continuous monitoring requirements, the number of these emission points that do not comply with the Section 61.93(b) requirements, and if possible, the cost for upgrades. Describe site periodic confirmatory measurement plans. Indicate the status of the quality assurance program described by Appendix B, Method 114.

The Naval Reactors Facility does not have any emission points that require continuous monitoring under Section 61.93(b).

Periodic confirmatory measurements were accomplished by calculating the maximum unabated emissions for radiological emission points at NRF to determine if continuous monitoring is required under Section 61.93. Periodic confirmatory measurements were also accomplished by use of calculations and samples to determine the actual emissions in 1997.

Though NRF does not require continuous monitoring, a quality assurance (QA) program is incorporated into the environmental monitoring program. The QA program includes equipment calibration, the use of blanks and known standards, and the annual review and validation of radioactive airborne emission data by independent peer reviewers. 
APPENDIX B

INEEL Research Center Report 


\section{Compliance With 40 CFR 61.94 For The INEEL Research Center}

40 CFR 61.94(a) Compliance with this standard is demonstrated by use of 40 CFR 61 Appendix E. A comparison of the January 1, 1997 inventory plus all receipts received during the calendar year with the Appendix E limits appears in Table B-1 (See section (b)(2)). This table shows the quantity of radioactive material possessed during the calendar year is less than the Appendix $\mathrm{E}$ limits.

40 CFR 61.94(b) In addition to paragraph (a), the annual report will include the following information:

40 CFR 61.94(b)(1) The name and location of the facility.

Idaho National Engineering and Environmental Laboratory (INEEL) Research Center (IRC) facilities are located on a partially developed 14.3-hectare (35.5-acre) plot on the north side of the City of Idaho Falls. Though programs and operations at the IRC are affiliated with the INEEL, the IRC is located within the city limits of Idaho Falls and is not contiguous with the INEEL site, whose nearest boundary is located approximately 22 miles west of Idaho Falls.

Facilities at the IRC include office, laboratory, and technical support buildings. The largest is a 3-story office building connected by an enclosed walkway to a 1-story laboratory building containing 66 laboratories. Other buildings at the IRC include the Research Office Building, Physics Building, Electric Vehicle Building, and Systems Analysis Facility.

40 CFR 61.94(b)(2) A list of the radioactive materials used at the facility.

See Table B-1.

40 CFR 61.94(b)(3) A description of the handling and processing that the radioactive materials undergo at the facility. 
The laboratory/office building is principally an experimental research facility dedicated to a wide range of research areas, including industrial microbiology; geochemistry; materials characterization; welding; ceramics; thermal fluids behavior; materials testing; nondestructive evaluation of materials using a standard industrial $x$-ray device, $x$-ray diffusion, and $\mathrm{x}$-ray fluorescence; analytical and environmental chemistry; and biotechnology. Sample analysis, including assay of biological samples for radioactive contamination, and other INEEL support functions are also conducted at IRC facilities.

\section{Table B-1 $\quad 40$ CFR 61 Appendix E Compliance Table}

\begin{tabular}{|c|c|c|c|}
\hline Radionnclide & $\begin{array}{c}\text { IRC Possession Quanttiy (CI) } \\
\text { Inchudes 1/197 Inventory Phus } \\
\text { All Materials Recelved in CY } \\
1997\end{array}$ & Physical State of Inventory & $\begin{array}{c}\text { Appendix E } \\
\text { Possession Quantity } \\
\text { Limit (C) }\end{array}$ \\
\hline $\mathrm{Ba}-133$ & $1.2 \mathrm{E}-06$ & Liquid/Powder & $4.9 \mathrm{E}-02$ \\
\hline C-14 & $2.4 \mathrm{E}-6$ & Liquid/Powder & $2.9 \mathrm{E}+02$ \\
\hline Cs-137 & $1.1 \mathrm{E}-07$ & Liquid/Powder & 2.3E-02 \\
\hline H-3 & 7.9E-07 & Liquid/Powder & $1.5 E+04$ \\
\hline $\mathrm{Ni}-63$ & $1.6 \mathrm{E}-06$ & Liquid/Powder & $1.4 \mathrm{E}+02$ \\
\hline P-32 & $1.51-06$ & Liquid/Powder & $1.7 \mathrm{E}+01$ \\
\hline $\mathrm{Pb}-210$ & $5.25-12$ & Liquid/Powder & 5.5E-02 \\
\hline Pu-239 & $9.0 \mathrm{E}-10$ & Liquid/Powder & $2.5 E-03$ \\
\hline S-35 & $1.5 E-06$ & Liquid/Powder & $7.5 \mathrm{E}+01$ \\
\hline U-234 & $4.56-10$ & Liquid/Powder & $7.6 \mathrm{E}-03$ \\
\hline U-235 & $5.7 \mathrm{E}-8$ & Liquid/Powder & $7.0 \mathrm{E}-03$ \\
\hline U-238 & 8.1E-07 & Liquid/Powder & $8.6 \mathrm{E}-03$ \\
\hline
\end{tabular}


40 CFR 61.94(b)(4) A list of the stacks or vents or other points where radioactive materials are released to the atmosphere.

Radiological releases from the IRC could arise from uncontrolled laboratory fumehoods within the facility. Exhaust from most of the fume hoods is released directly to the outside atmosphere via the heat recovery fan (HRF) system of the facility HVAC system. The HRF system exhausts to the outside via three vertical vents on the north side of the mechanical penthouse on top of the IRC laboratory building. Stack height of these vents is $7.6 \mathrm{~m}$ ( $25 \mathrm{f}$ ). The exhausts from other fume hoods (not exhausted to the HRF) are released to the atmosphere via a $2.1-\mathrm{m}(7.0-\mathrm{ft})$ stack above the roof or two 8.5 $\mathrm{m}(28 \mathrm{ft})$ stacks above the roof.

40 CFR 61.94(b)(5) A description of the effluent controls that are used on each stack, vent, or other release point and an estimate of the efficiency of each control device.

None. There is no effluent control equipment associated with any of the IRC's release points.

40 CFR 61.94(b)(6) Distances from the points of release to the nearest residence, school, business or office and the nearest farms producing vegetables, milk, and meat.

Consistent with 40 CFR 61 Appendix E no person lives within 10 meters of the IRC and no milk, meat, or vegetables are produced within 100 meters of the IRC.

40 CFR 61.94(b)(7) The values used for all other user-supplied input parameters for the computer models (e.g., meteorological data) and the source of these data.

Not Applicable. 40 CFR 61 Appendix E used for compliance. 
40 CFR 61.94(b)(8) A brief description of all construction and modifications that were completed in the calendar year for which the report is prepared, but for which the requirement to apply for approval to construct or modify was waived under $\$ 61.96$ and associated documentation developed by DOE to support the waiver.

None. 


\title{
APPENDIX C
}

\author{
1997 Meteorology Data
}

For CAP-88 Computer Code 


\section{STAR File Format}

This is a STability ARray (STAR) file. It shows the frequencies of occurrence that the wind is blowing FROM a particular direction, at a particular stability, at a particular speed. The frequencies are in $\mathbf{x} . \times x \times x x$ format, unspaced. The format is:

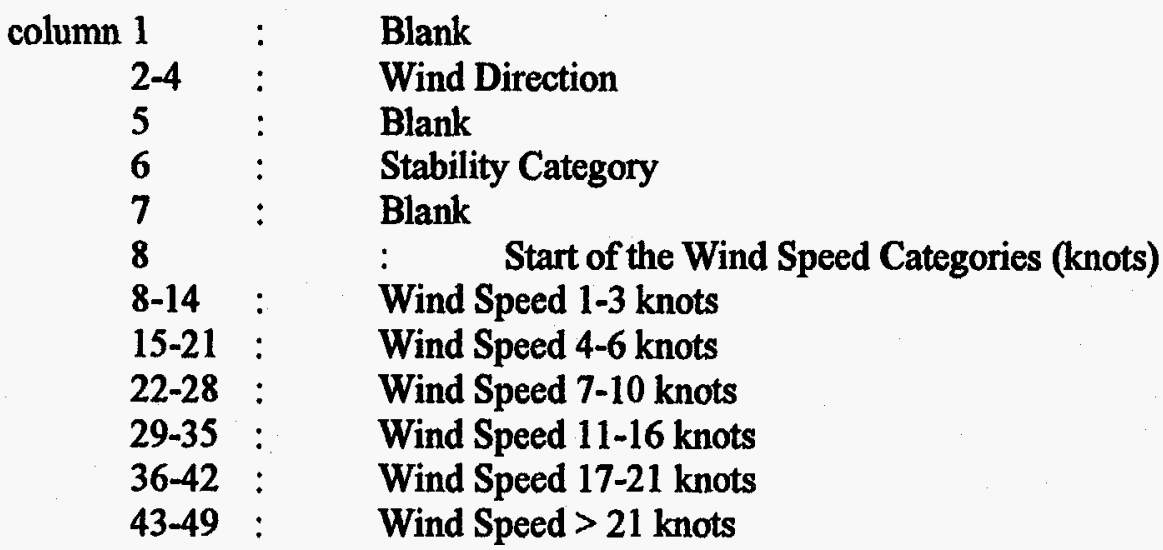

N A 0.000080 .000660 .000000 .000000 .000000 .00000 NNE A 0.000160 .000330 .000000 .000000 .000000 .00000

NE A 0.000160 .000160 .000000 .000000 .000000 .00000

ENE A 0.000080 .000000 .000000 .000000 .000000 .00000 


\section{Table C-1. STAR File Used for ANL Ground Level Releases}

N 0.001740 .002910 .000000 .000000 .000000 .00000 HNE A 0.002560 .005000 .000000 .000000 .000000 .00000 NE A 0.001740 .004300 .000000 .000000 .000000 .00000 ENE A 0.001160 .003490 .000000 .000000 .000000 .00000 E A 0.001400 .001860 .000000 .000000 .000000 .00000 ESE A 0.000810 .001980 .000000 .000000 .000000 .00000 SE A 0.000120 .001160 .000000 .000000 .000000 .00000 SSE A 0.000230 .002680 .000000 .000000 .000000 .00000 $S$ A 0.000350 .002910 .000000 .000000 .000000 .00000 SSW A 0.000700 .003140 .000000 .000000 .000000 .00000 SW A 0.001160 .004420 .000000 .000000 .000000 .00000 WSW A 0.001740 .003960 .000000 .000000 .000000 .00000 W A 0.002210 .003840 .000000 .000000 .000000 .00000 WNW A 0.002090 .003020 .000000 .000000 .000000 .00000 NW A 0.002440 .003020 .000000 .000000 .000000 .00000 NNW A 0.002790 .002560 .000000 .000000 .000000 .00000

N B 0.000230 .001740 .000230 .000000 .000000 .00000 NNE B 0.000000 .002330 .000700 .000000 .000000 .00000 NE B 0.000470 .001510 .000700 .000000 .000000 .00000 ENE B 0.000350 .000930 .000230 .000000 .000000 .00000 E B 0.000000 .000350 .000700 .000000 .000000 .00000 SE B 0.000120 .000470 .000000 .000000 .000000 .00000 SE 0.000000 .000470 .000580 .000000 .000000 .00000 SSE B 0.000000 .000700 .000700 .000000 .000000 .00000 $S$ B 0.000120 .001160 .001630 .000000 .000000 .00000 SSW B 0.000000 .001400 .001510 .000000 .000000 .00000 SW B 0.000120 .002560 .002330 .000000 .000000 .00000 WSW B 0.000350 .001630 .002330 .000000 .000000 .00000 W B 0.000000 .000700 .000930 .000000 .000000 .00000 W B 0.000000 .000700 .000930 .000000 .000000 .00000 NIT B 0.000000 .000470 .000470 .000 NNW B 0.000000 .000930 .000230 .000000 .000000 .00000 N C 0.000000 .001160 .001280 .000000 .000000 .00000 NNE C 0.000470 .003140 .002090 .000120 .000000 .00000 $\mathrm{NE} C \mathrm{C} 0.000470 .003610 .001400 .000120 .000000 .00000$ ENE C 0.000230 .001050 .001280 .000120 .000000 .00000 E C 0.000120 .000470 .000230 .000120 .000000 .00000 ESE C 0.000000 .000120 .000230 .000120 .000000 .00000 SE C 0.000120 .000120 .000470 .000120 .000000 .00000 SSE C 0.000000 .000580 .001860 .000120 .000000 .00000 S C 0.000000 .000470 .004070 .000470 .000000 .00000 SSW C 0.000120 .001510 .007210 .000700 .000000 .00000 SW C 0.000230 .001860 .006630 .001280 .000000 .00000 WSW C 0.000120 .002090 .002440 .000230 .000000 .00000 W c 0.000120 .000470 .002210 .000000 .000000 .00000 WNW C 0.000000 .000120 .001280 .000120 .000000 .00000 NW C 0.000230 .000700 .001050 .000350 .000000 .00000 NNW C 0.000230 .000580 .000810 .000120 .000000 .00000
N D 0.000600 .006750 .005350 .005930 .002910 .00128 NNE D 0.002500 .022570 .016050 .015940 .004650 .00256 NE D 0.001790 .017100 .015470 .008610 .001160 .00035 ENE D 0.000710 .008720 .005820 .001510 .000120 .00000 E D 0.000710 .003140 .002210 .000350 .000000 .00000 ESE D 0.000120 .001280 .001630 .000470 .000120 .00000 SE D 0.000120 .001160 .003720 .001050 .000000 .00000 SE 0.000240 .003370 .013490 .006280 .000930 .00000 $S$ D 0.000950 .007450 .018030 .018500 .004770 .00047 SSW D 0.000710 .008380 .021410 .040720 .013260 .00279 SW D 0.000240 .006050 .021520 .042690 .029780 .01128 WSW D 0.000000 .004540 .011170 .016050 .009660 .01221 W D 0.000120 .001050 .003490 .001740 .000470 .00012 WNW D 0.000000 .001630 .002440 .001630 .000120 .00000 NW D 0.000240 .002090 .003960 .002680 .000230 .00012 NNW D 0.000830 .002560 .004540 .003490 .001280 .00012

N E 0.000350 .003370 .002440 .000000 .000000 .00000 NNE E 0.001530 .005820 .005230 .000000 .000000 .00000 NE E 0.000820 .008380 .005820 .000000 .000000 .00000 ENE E 0.001300 .008140 .003610 .000000 .000000 .00000 E E 0.000000 .003490 .003020 .000000 .000000 .00000 ESE E 0.000710 .000930 .001160 .000000 .000000 .00000 SE E 0.000350 .000930 .001400 .000000 .000000 .00000 SSE E 0.000940 .003140 .017920 .000000 .000000 .00000 $S$ E 0.000940 .006860 .004190 .000000 .000000 .00000 SSW E 0.000940 .005230 .006510 .000000 .000000 .00000 SW E 0.000240 .003260 .005350 .000000 .000000 .00000 WSW E 0.000000 .003020 .002680 .000000 .000000 .00000 W E 0.000000 .001860 .000350 .000000 .000000 .00000 WNW E 0.000240 .001400 .000580 .000000 .000000 .00000 NNW E 0.000350 .002560 .002910 .000000 .000000 .00000 N F 0.006200 .005820 .000000 .000000 .000000 .00000 NNE F 0.008230 .007330 .000000 .000000 .000000 .00000 NE F 0.007030 .010940 .000000 .000000 .000000 .00000 ENE F 0.008110 .006510 .000000 .000000 .000000 .00000 E F 0.007990 .004070 .000000 .000000 .000000 .00000 SE F 0.004650 .002210 .000000 .000000 .000000 .00000 SSE F 0.004650 .005000 .000000 .000000 .000000 .00000 S F 0.006320 .005700 .000000 .000000 .000000 .00000 SSW F 0.006200 .005580 .000000 .000000 .000000 .00000 SW F 0.005010 .007330 .000000 .000000 .000000 .00000 WSW F 0.005480 .005820 .000000 .000000 .000000 .00000 W F 0.002270 .003260 .000000 .000000 .000000 .00000 NW F 0.003460 .002910 .000000 .000000 .000000 .00000 NNW E 0.005130 .004070 .000000 .000000 .000000 .00000 
Table C-2. STAR File Used for ANL Elevated Releases

N A 0.001290 .002220 .000000 .000000 .000000 .00000 NNE A 0.001060 .003730 .000120 .000000 .000000 .00000 NE A 0.001880 .003150 .000000 .000000 .000000 .00000 ENE A 0.000470 .003150 .000000 .000000 .000000 .00000 E A 0.001290 .002100 .000120 .000000 .000000 .00000 ESE A 0.000350 .001400 .000000 .000000 .000000 .00000 SE A 0.000350 .001750 .000120 .000000 .000000 .00000 SSE A 0.000230 .002220 .000120 .000000 .000000 .00000 A A 0.000470 .002330 .000230 .000000 .000000 .00000 SSW A 0.001410 .002680 .000120 .000000 .000000 .00000 SW A 0.000940 .003620 .000350 .000120 .000000 .00000 WSW A 0.001880 .003030 .000120 .000000 .000000 .00000 W A 0.001530 .003150 .000000 .000000 .000000 .00000 WNW A 0.001170 .002220 .000000 .000000 .000000 .00000 NW A 0.002110 .001520 .000230 .000000 .000000 .00000 N B 0.000000 .000700 .000120 .000000 .000000 .00000 NNE B 0.000230 .001520 .001050 .000000 .000000 .00000 NE B 0.000120 .001280 .000930 .000000 .000000 .00000 ENE B 0.000120 .001170 .000350 .000000 .000000 .00000 E B 0.000000 .000120 .000930 .000000 .000000 .00000 ESE B 0.000000 .000120 .000230 .000000 .000000 .00000 SE B 0.000000 .000000 .000930 .000000 .000000 .00000 SSE B 0.000000 .000470 .001400 .000000 .000000 .00000 S B 0.000000 .000120 .001870 .000000 .000000 .00000 SSW B 0.000120 .000700 .002100 .000120 .000000 .00000 SW B 0.000000 .001750 .002680 .000000 .000000 .00000 WSW B 0.000230 .000580 .002330 .000000 .000000 .00000 W B 0.000120 .000820 .001280 .000000 .000000 .00000 WN 0.000120 .000580 .000350 .000000 .000000 .00000 NW 0.000230 .000470 .000700 .000000 .000000 .00 NNW B 0.000000 .000820 .000580 .000000 .000000 .00000 N C 0.000350 .000820 .000700 .000580 .000000 .00000 NNE C 0.000820 .001870 .001870 .000700 .000000 .00000 NE C 0.000350 .002920 .001400 .000350 .000000 .00000 ENE C 0.000120 .000700 .000820 .000230 .000000 .00000 E C 0.000230 .000350 .000230 .000120 .000000 .00000 E C 0.000000 .000230 .000120 .000230 .000000 .00000 SE C 0.000000 .000230 .000230 .000350 .000000 .00000 SSE C 0.000000 .000230 .000820 .000930 .000000 .00000 S C 0.000000 .000470 .002450 .001630 .000000 .00000 SsW C 0.000000 .000930 .005020 .002570 .000000 .00000 SW C 0.000120 .001170 .003380 .003030 .000000 .00000 WSW C 0.000120 .000470 .001870 .000930 .000120 .00000 W c 0.000000 .000820 .001050 .000580 .000000 .00000 WIT c 0.000000 .000820 .001050 .000580 .000000 .00000 NW C 0.000350 .000230 .000930 .000470 .000000 .00000 NNW C 0.000120 .000470 .000470 .000580 .000000 .00000
N D 0.000350 .006530 .005130 .005130 .005020 .00397 NNE D 0.001050 .019360 .018190 .007230 .012480 .00898 NE D 0.001630 .011900 .019830 .010150 .005950 .00350 ENE D 0.000700 .003270 .005020 .001870 .001400 .00058 E D 0.000000 .001400 .001400 .001870 .000470 .00012 ESE D 0.000000 .001280 .001400 .000700 .000350 .00012 SE D 0.000000 .000580 .001400 .001280 .000580 .00070 SSE D 0.000000 .001520 .002680 .004550 .003850 .00175

$S$ D 0.000000 .000580 .005480 .013760 .012600 .00688 SsW D 0.000120 .002330 .010380 .024380 .020760 .02251 SW D 0.000230 .004200 .010850 .022630 .036160 .04059 WSW D 0.000230 .003970 .007580 .012360 .014580 .02753 W D 0.000230 .001630 .003030 .004200 .002100 .00058 WNW D 0.000230 .002680 .002450 .002570 .000700 .00012 NW D 0.000230 .002330 .003150 .002800 .002920 .00128 NNW D 0.000470 .002680 .003500 .003730 .003500 .00117 $N E 0.000240 .003270 .005600 .005020 .001750 .00012$ NNE E 0.000720 .009680 .011430 .006760 .003270 .00000 NE E 0.000720 .005950 .015160 .010850 .004550 .00000 ENE E 0.000600 .003380 .006530 .003620 .000930 .00000 E E 0.000000 .000930 .001280 .000470 .000120 .00000 ESE E $0.000120 .001050 .001520,000700.000000 .00000$ SE E 0.000120 .000230 .001050 .001050 .000350 .00000 SSE E 0.000000 .000700 .002100 .003380 .001050 .00000 $S$ E 0.000000 .001400 .003730 .008510 .002220 .00000 SSW E 0.000120 .001170 .004670 .010150 .002920 .00000 SW E 0.000240 .001750 .004670 .009910 .003970 .00000 WSW E 0.000360 .001520 .005710 .005480 .002100 .00000 $W \mathrm{E} 0.000360 .001400 .002330 .001750 .000230 .00000$ NW E 0.000360 .002220 .004080 .001400 .001050 .00000 NNW E 0.000720 .004670 .003270 .004200 .001050 .00000 N F 0.001530 .005250 .002100 .000350 .000000 .00000 NNE E 0.002470 .004430 .004670 .001870 .000120 .00000 NE F 0.001650 .003150 .003500 .002920 .000000 .00000 ENE F 0.001300 .003150 .001280 .000470 .000120 .00000 E 0.001770 .001630 .000470 .000000 .000000 .00000 ESE 0.000940 .001630 .000230 .000120 .000000 .00000 SE F 0.000940 .001630 .000700 .000120 .000000 .00000 SSE F 0.001060 .001630 .000230 .000000 .000000 .00000 S F 0.000710 .001630 .000820 .000350 .000000 .00000 SSW F 0.001650 .001520 .000820 .000820 .000000 .00000 SW F 0.001650 .002800 .001520 .000580 .000000 .00000 WSW F 0.001180 .003150 .001050 .000350 .000000 .00000

W F 0.001300 .003150 .001170 .000230 .000000 .00000 WNW F 0.002240 .002800 .001170 .000120 .000000 .00000 NW F 0.002000 .003620 .002100 .000120 .000000 .00000 NNW F 0.001880 .002680 .001630 .001170 .000120 .00000 


\section{Table C-3. STAR File Used for CFA (and RWMC) Ground Level Releases}

N A 0.002700 .001720 .000000 .000000 .000000 .00000 NNE A 0.002110 .003100 .000000 .000000 .000000 .00000 NE A 0.001990 .006090 .000000 .000000 .000000 .00000 ENE A 0.001760 .004940 .000000 .000000 .000000 .00000 A 0.002460 .003450 .000000 .000000 .000000 .00000 ESE A 0.001640 .003560 .000000 .000000 .000000 .00000 SE A 0.001410 .003220 .000000 .000000 .000000 .00000 SSE A 0.001520 .006320 .000000 .000000 .000000 .00000 S A 0.001520 .004370 .000000 .000000 .000000 .00000 SSW A 0.001170 .003790 .000000 .000000 .000000 .00000 SW A 0.000590 .004370 .000000 .000000 .000000 .00000 WS A 0.000590 .002300 .000000 .000000 .000000 .00000

W A 0.000700 .001610 .000000 .000000 .000000 .00000 WNW A 0.000590 .001150 .000000 .000000 .000000 .00000 NW A $0.000230 .001150 .000000,000000.000000 .00000$ NNW A 0.001410 .001490 .000000 .000000 .000000 .00000 N B 0.000230 .001380 .000340 .000000 .000000 .00000 NNE B 0.000460 .001380 .000460 .000000 .000000 .00000 NE 80.000690 .002870 .000570 .000000 .000000 .00000 ENE 0.000230 .000920 .000000 .000000 .000000 .00000 E B 0.000110 .000920 .000920 .000000 .000000 .00000 ESE B 0.000000 .000000 .000570 .000000 .000000 .00000 SE B 0.000110 .000920 .000110 .000000 .000000 .00000 SSE B 0.000110 .001610 .000570 .000000 .000000 .00000 S B 0.000110 .001150 .001030 .000000 .000000 .00000 SSW B 0.000230 .002530 .002640 .000000 .000000 .00000 SW 0.000230 .001490 .002640 .000000 .000000 .00000 WSW B 0.000110 .001150 .001380 .000000 .000000 .00000 B 0.000000 .000690 .001030 .000000 .000000 .00000 WNW B 0.000110 .000110 .000570 .000000 .000000 .00000 NW B 0.000000 .000230 .000340 .000000 .000000 .00000 NNW B 0.000000 .000800 .000460 .000000 .000000 .00000 N C 0.000000 .000920 .000920 .000110 .000000 .00000 NNE C 0.000390 .002410 .002180 .000000 .000000 .00000 NE C 0.000390 .003100 .002640 .000110 .000000 .00000 ENE C 0.000000 .001380 .001610 .000000 .000000 .00000 E C 0.000000 .000230 .000800 .000000 .000000 .00000 ESE C 0.000000 .000230 .000690 .000110 .000000 .00000 SE C 0.000130 .000570 .000340 .000000 .000000 .00000 SSE C 0.000000 .000690 .001030 .000110 .000000 .00000 S. C 0.000000 .000800 .002760 .000230 .000000 .00000 SSW C 0.000130 .001030 .007240 .001030 .000000 .00000 SW C 0.000130 .000460 .008960 .001260 .000000 .00000 WSW C 0.000000 .000690 .004600 .001030 .000000 .00000 W C 0.000000 .000460 .001840 .000230 .000000 .00000 WNW C 0.000390 .000460 .000690 .000230 .000000 .00000 NW C 0.000000 .000110 .000570 .000110 .000000 .00000 NNW C 0.000130 .000000 .000800 .000110 .000000 .00000
N D 0.001170 .007470 .004370 .006670 .002530 .00046 NNE D 0.002100 .030570 .022640 .008850 .002070 .00207 NE D 0.000470 .015170 .023790 .018040 .003100 .00161 ENE D 0.000120 .002410 .004020 .004140 .000000 .00000 E D 0.000230 .001260 .001150 .000110 .000000 .00000 TSE 0.000000 .000920 .000920 .000230 .000000 .00000 SE D 0.000000 .002410 .001840 .000460 .000000 .00000 SSE D 0.000000 .004600 .003450 .002070 .000110 .00000 S D 0.000350 .004370 .010110 .005750 .001490 .00011 SSW D 0.000230 .005170 .015280 .013330 .004250 .00057 SW D 0.000350 .005060 .026660 .055620 .032980 .01218 WSW D 0.000580 .007010 .024250 .041370 .019770 .01896 W D 0.000000 .006550 .008730 .004370 .001380 .00023 WNW D 0.000350 .004020 .003910 .002410 .000340 .00011 NW D 0.000820 .002870 .001840 .001840 .000800 .00011 NNW D 0.000820 .003450 .001490 .001950 .000230 .00011 N E 0.001630 .007240 .003100 .000000 .000000 .00000 NNE E 0.001170 .014130 .011950 .000000 .000000 .00000 NE E 0.001170 .006890 .007700 .000000 .000000 .00000 ENE 0.000120 .002760 .003200 .000000 .000000 .00000 B 0.000120 .0

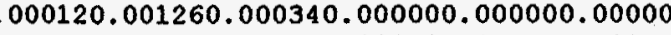
ESE E 0.000230 .000690 .000340 .000000 .000000 .00000 SE E 0.000000 .001150 .000570 .000000 .000000 .00000 SSE E $0.000000 .002990 .002070 .000000,000000.00000$ S E 0.000350 .003910 .003560 .000000 .000000 .00000 SSW E 0.000000 .002760 .002070 .000000 .000000 .00000 0.009420 .000000 .000000 .00000 . WNW E 0.000700 .004140 .000460 .000000 .000000 .00000 NW E 0.000700 .004020 .000570 .000000 .000000 .00000 NNW E 0.000700 .005290 .000920 .000000 .000000 .00000 N F 0.004850 .007930 .000000 .000000 .000000 .00000 NNE $\quad 0.003580 .009650 .000000 .000000 .000000 .00000$ ENE F 0.002080 .002300 .000000 .000000 .000000 .00000 E F 0.002080 .003100 .000000 .000000 .000000 .00000 SE F 0.002200 .003680 .000000 .000000 .000000 .00000 SSE F 0.003810 .005400 .000000 .000000 .000000 .00000 S F 0.003580 .006440 .000000 .000000 .000000 .00000 SSW F 0.004390 .007580 .000000 .000000 .000000 .00000 SW E $0.003010 .007350,000000.000000 .000000 .00000$ WSW F 0.003700 .006780 .000000 .000000 .000000 .00000 W F 0.003350 .007580 .000000 .000000 .000000 .00000 WNW F 0.003930 .007700 .000000 .000000 .000000 .00000 NW F 0.005900 .007120 .000000 .000000 .000000 .00000 NNW F 0.006360 .008850 .000000 .000000 .000000 .00000 
Table C-4. STAR File Used for ICPP Ground Level Releases

N A 0.000810 .000800 .000000 .000000 .000000 .00000 NNE A 0.001610 .003560 .000000 .000000 .000000 .00000 NE A 0.002540 .006650 .000000 .000000 .000000 .00000 ENE A 0.003460 .005850 .000000 .000000 .000000 .00000

E A 0.001960 .004250 .000000 .000000 .000000 .00000 ESE A 0.001960 .003670 .000000 .000000 .000000 .00000 SE A 0.001840 .003440 .000000 .000000 .000000 .00000 SSE A 0.000920 .005850 .000000 .000000 .000000 .00000 $S$ A 0.002070 .006420 .000000 .000000 .000000 .00000 SSW A 0.001500 .005620 .000000 .000000 .000000 .00000 SW A 0.001150 .004470 .000000 .000000 .000000 .00000 WSW A 0.001040 .002520 .000000 .000000 .000000 .00000 W A 0.001380 .000800 .000000 .000000 .000000 .00000 WNW A 0.000810 .000570 .000000 .000000 .000000 .00000 NW A 0.000580 .001030 .000000 .000000 .000000 .00000 NNW A 0.000580 .001150 .000000 .000000 .000000 .00000

N B 0.000230 .000570 .000340 .000000 .000000 .00000 NNE B 0.000110 .001840 .000570 .000000 .000000 .00000 NE B 0.001030 .003210 .000690 .000000 .000000 .00000 ENE B 0.000230 .001150 .001260 .000000 .000000 .00000 E B 0.000000 .001260 .000570 .000000 .000000 .00000 ESE B 0.000000 .000460 .000110 .000000 .000000 .00000 SE B 0.000230 .000460 .000340 .000000 .000000 .00000 SSE B 0.000110 .000920 .000690 .000000 .000000 .00000

$S$ B 0.000230 .003900 .001150 .000000 .000000 .00000 B B 0.000230 .003900 .001150 .000000 .000000 .00000 SW B 0.000000 .002180 .002520 .000000 .000000 .00000 WSW B 0.000000 .000460 .001150 .000000 .000000 .00000 W B 0.000000 .000460 .000460 .000000 .000000 .00000 WNW B 0.000000 .000460 .000340 .000000 .000000 .00000 NW B 0.000000 .000460 .000570 .000000 .000000 .00000 NNW B 0.000110 .000460 .000110 .000000 .000000 .00000 N C 0.000230 .001490 .001260 .000230 .000000 .00000 INE C 0.000570 .002640 .001840 .000340 .000000 .00000 $\mathrm{NE}$ C 0.000800 .004590 .002750 .000110 .000000 .00000 ENE C 0.000110 .001260 .002180 .000000 .000000 .00000 E C 0.000000 .000340 .000460 .000000 .000000 .00000 ESE C 0.000000 .000110 .000230 .000000 .000000 .00000 SE C 0.000110 .000230 .000460 .000000 .000000 .00000 SSE C 0.000000 .000920 .001030 .000110 .000000 .00000 s c 0.000110 .001150 .002750 .000460 .000000 .00000 SSW C 0.000110 .001490 .008720 .001150 .000000 .00000 SW C 0.000110 .001260 .006650 .002070 .000000 .00000 WSW C 0.000000 .000800 .003210 .000230 .000000 .00000 W C 0.000000 .000230 .001380 .000230 .000000 .00000 WW C 0.000000 .000000 .000690 .000000 .000000 .00000 NW 0.000000 .000110 .001030 .000110 .000000 .00000 NNW C 0.000230 .000110 .001150 .000340 .000000 .00000
N D 0.001390 .009870 .006650 .007800 .003900 .00034 NNE D 0.001390 .019960 .016640 .006880 .001610 .00161 NE D 0.001390 .024780 .027540 .014570 .001950 .00080 ENE D 0.000700 .002750 .007800 .002410 .000570 .00034 E D 0.000000 .001150 .001490 .000340 .000000 .00000 ESE D 0.000460 .001030 .000460 .000230 .000000 .00000 SE D 0.000000 .000800 .001030 .000230 .000000 .00000 SSE D 0.000120 .003440 .003900 .000340 .000110 .00000 S D 0.000700 .006540 .007800 .004820 .001490 .00011 SSW D 0.000700 .008600 .023290 .027880 .008030 .00149 SW D 0.000460 .012050 .036480 .058050 .031550 .01721 WSW D 0.000460 .007800 .013880 .010780 .007110 .00390 W D 0.000580 .002180 .003210 .002290 .000920 .00000 WNW D 0.000580 .000690 .002070 .001030 .000230 .00000 NW D 0.000000 .000800 .001840 .000920 .000230 .00000 NNW D 0.000230 .001380 .003900 .006200 .001490 .00034 N E 0.001620 .007570 .002410 .000000 .000000 .00000 NNE E 0.001390 .014570 .007230 .000000 .000000 .00000 NE E 0.001390 .010440 .008720 .000000 .000000 .00000 ENE E 0.000460 .004590 .004020 .000000 .000000 .00000 E E 0.000230 .001380 .000690 .000000 .000000 .00000 ESE E 0.000000 .001720 .000110 .000000 .000000 .00000 SE E 0.000120 .001260 .000460 .000000 .000000 .00000 SSE E 0.000230 .003900 .001950 .000000 .000000 .00000 $S E 0.000580 .004700 .003790 .000000 .000000 .00000$ SSW E 0.000810 .007460 .005620 .000000 .000000 .00000 SW E 0.000230 .008600 .012280 .000000 .000000 .00000 WSW E 0.000700 .005740 .003670 .000000 .000000 .00000 W E 0.000580 .002520 .000920 .000000 .000000 .00000 WNW E 0.000460 .001380 .000340 .000000 .000000 .00000 NW E 0.000350 .001150 .000230 .000000 .000000 .00000 NNW 0.000330 .002290 .000460 .000000 .000000 .00000 N F 0.008940 .008490 .000000 .000000 .000000 .00000 NNE F 0.005250 .010330 .000000 .000000 .000000 .00000 NE E 0.007270 .006880 .000000 .000000 .000000 .00000 ENE F 0.002980 .002870 .000000 .000000 .000000 .00000 E F 0.002030 .002980 .000000 .000000 .000000 .0000 ESE E 0.002620 .002870 .000000 .000000 .000000 .00000 SE F 0.003460 .003330 .000000 .000000 .000000 .00000 SSE F 0.002860 .005160 .000000 .000000 .000000 .00000 S 0.005480 .010100 .000000 .000000 .000000 .00000 SSW 0.005840 .009410 .000000 .000000 .000000 .00000 SW F 0.006680 .007690 .000000 .000000 .000000 .00000 WSW F 0.005010 .007920 .000000 .000000 .000000 .00000 W F 0.004650 .006310 .000000 .000000 .000000 .00000 WNF 0.005130 .005160 .000000 .000000 .000000 .00000 NW F 0.004290 .003790 .000000 .000000 .000000 .00000 NNW F 0.006440 .005970 .000000 .000000 .000000 .00000 
Table C-5. STAR File Used for ICPP Elevated Releases

N A 0.000820 .000690 .000120 .000000 .000000 .00000 NNE A 0.000930 .002780 .000000 .000000 .000000 .00000 NE $A \quad 0.001170 .004160 .000120 .000000 .000000 .00000$ ENE A 0.001400 .005090 .000230 .000000 .000000 .00000 E A 0.001050 .003120 .000230 .000000 .000000 .00000 ESE A 0.000930 .003930 .000230 .000000 .000000 .00000 SE A 0.001050 .002890 .000000 .000000 .000000 .00000 SSE A 0.001170 .005780 .000120 .000000 .000000 .00000 S A 0.001050 .005320 .000120 .000000 .000000 .00000 SSW A 0.001400 .004050 .000230 .000000 .000000 .00000 SW A 0.001400 .003120 .000580 .000000 .000000 .00000 WSW A 0.000350 .001620 .000350 .000000 .000000 .00000 W A 0.001050 .001500 .000120 .000000 .000000 .00000 WNA A 0.000350 .001270 .000000 .000000 .000000 .00000 NW A 0.000470 .000810 .000120 .000000 .000000 .00000 NNW A 0.000820 .000810 .000120 .000000 .000000 .00000 N B 0.000120 .000120 .000350 .000000 .000000 .00000 NNE B 0.000360 .001730 .000580 .000000 .000000 .00000 NE B 0.000360 .001500 .001040 .000120 .000000 .00000 ENE B 0.000360 .001730 .001620 .000000 .000000 .00000 E B 0.000120 .000460 .000810 .000000 .000000 .00000 ESE B 0.000120 .000350 .000230 .000000 .000000 .00000 SE B 0.000120 .001040 .000460 .000000 .000000 .00000 SSE B 0.000240 .000580 .000930 .000000 .000000 .00000 S B 0.000120 .001970 .001850 .000000 .000000 .00000 SSW B 0.000360 .001500 .003590 .000000 .000000 .00000 SW B 0.000000 .000930 .003470 .000000 .000000 .00000 WSW B 0.000000 .000350 .001040 .000000 .000000 .00000 W B 0.000000 .000230 .000810 .000000 .000000 .00000 WNW B 0.000120 .000000 .000580 .000000 .000000 .00000 NW B 0.000000 .000230 .000580 .000000 .000000 .00000 NNW B 0.000000 .000120 .000580 .000000 .000000 .00000 N C 0.000120 .000460 .000580 .000460 .000000 .00000 NNE C 0.000230 .001850 .000810 .001160 .000000 .00000 NE C 0.000460 .003120 .002080 .000230 .000000 .00000 ENE C 0.000350 .001160 .001730 .000230 .000000 .00000 E C 0.000000 .000350 .000580 .000230 .000000 .00000 ESE C 0.000000 .000460 .000460 .000000 .000000 .0000 SE C 0.000000 .000580 .000350 .000350 .000000 .00000 SSE C 0.000120 .000580 .000810 .000350 .000000 .00000 S C 0.000120 .001270 .002310 .001390 .000000 .00000 STW 0.000120 .001500 .005200 .004510 .000000 .00000 SW C 0.000000 .000690 .003930 .003590 .000120 .00000 WSW C 0.000000 .000930 .001620 .001620 .000000 .00000 W C 0.000350 .000120 .001390 .000690 .000000 .00000 WNW C 0.000000 .000000 .000460 .000230 .000000 .00000 NW C 0.000000 .000230 .000460 .000690 .000000 .00000 NNW C 0.000000 .000120 .000810 .000810 .000120 .00000
N D 0.000360 .002200 .003700 .004510 .006010 .00497 NNE D 0.001320 .011220 .016420 .010410 .004390 .00324 NE D 0.001680 .021280 .033650 .017810 .010520 .00173 ENE D 0.000360 .004970 .004630 .003010 .002310 .00104 D 0.000240 .000460 .001160 .000580 .000350 .00000 ESE D 0.000240 .000810 .000580 .000350 .000230 .00000 SE D 0.000120 .001040 .000810 .000580 .000000 .00012 SSE D 0.000120 .003120 .003240 .001160 .000000 .00012 S D 0.000360 .004740 .006590 .004970 .003240 .00139 SSW D 0.000240 .004510 .011100 .020470 .015270 .00671 SW D 0.000360 .003590 .016310 .036310 .052040 .04672 T. 0.000000 .0004 W D 0.00000 .000 WNW D 0.000120 .000230 .001160 .001620 .000690 .00035 NW D 0.000240 .000460 .001850 .001500 .000230 .00035 NNW D 0.000120 .000810 .001730 .003470 .005440 .00278 N E 0.000350 .002430 .002430 .002540 .000580 .00000 NNE E 0.001300 .007520 .014570 .009710 .000930 .00000 NE E 0.000470 .009830 .022320 .026370 .004630 .00035 ENE 0.000590 .002780 .003120 .002540 .001500 .00012 E E 0.000240 .001850 .001040 .000120 .000000 .00000 E E 0.000240 .001850 .001040 .000120 .000000 .00000 ESE E 0.000240 .001270 .000810 .000000 .000000 .00000 SE E 0.000120 .000810 .000690 .000460 .000120 .00000 SSE E 0.000240 .002310 .002780 .001160 .000000 .00000 S E 0.000120 .002660 .003930 .002540 .000580 .00000 SSW E 0.000350 .003120 .004050 .004390 .001040 .00000 SW $E 0.000350 .003240 .007750 .015380 .003700 .00000$ TH 0.000350 .003240 .007750 .015380 .00 W E 0.000350 .001850 .003240 .008330 .000930 .00000 W E 0.000000 .000930 .001850 .001270 .000000 .00000 WNW E 0.000350 .000460 .000930 .000230 .000000 .00000 NNW E 0.000240 .000690 .001270 .000810 .000350 .00000 N F 0.002380 .003820 .001730 .000000 .000000 .00000 NNE F 0.003580 .005780 .007170 .001500 .000120 .00000 NE F 0.001190 .004510 .001620 .000460 .000000 .00000 E F 0.002620 .003240 .001500 .000000 .000000 .00000 ESE F 0.001310 .003120 .000810 .000000 .000000 .00000 SE F 0.002380 .001730 .000230 .000000 .000000 .00000 SSE F 0.001790 .001500 .000460 .000120 .000000 .00000 S F 0.001550 .003590 .000580 .000000 .000000 .00000 SSW F 0.001430 .003930 .001970 .000000 .000000 .00000 SW 0.001430 . WSW E 0.001430 .002660 .001970 .000000 .000000 .00000 W E 0.002150 .003120 .001390 .000000 .000000 .00000 WNW F 0.001790 .002200 .001390 .000000 .000000 .00000 NW E 0.001310 .003010 .001500 .000000 .000000 .00000 NNW F 0.001190 .003820 .001040 .000120 .000000 .00000 
Table C-6. STAR File Used for NRF Ground Level Releases

N A 0.002210 .002530 .000000 .000000 .000000 .00000 NNE A 0.001980 .003330 .000000 .000000 .000000 .00000 NE A 0.001980 .005630 .000000 .000000 .000000 .00000 ENE A 0.001630 .006550 .000000 .000000 .000000 .00000

E A 0.001160 .004250 .000000 .000000 .000000 .00000 ESE A 0.001980 .003910 .000000 .000000 .000000 .00000 SE A 0.002100 .003680 .000000 .000000 .000000 .00000 SSE A 0.001630 .003330 .000000 .000000 .000000 .00000 $S$ A 0.001510 .005750 .000000 .000000 .000000 .00000 SSW A 0.001160 .004940 .000000 .000000 .000000 .00000 SW A 0.001160 .002870 .000000 .000000 .000000 .00000 WSW A 0.001280 .002070 .000000 .000000 .000000 .00000 W A 0.001510 .002530 .000000 .000000 .000000 .00000 WNW A 0.001160 .002070 .000000 .000000 .000000 .00000 NW A 0.001630 .001720 .000000 .000000 .000000 .00000 NNW A 0.001630 .002300 .000000 .000000 .000000 .00000

N B 0.000340 .001150 .000230 .000000 .000000 .00000 NNE B 0.000690 .001720 .000800 .000000 .000000 .00000 NE $B \quad 0.000570 .004370 .001030 .000000 .000000 .00000$ ENE 80.000340 .002640 .001490 .000000 .000000 .00000 E B 0.000340 .000690 .000340 .000000 .000000 .00000 ESE B 0.000000 .000690 .000460 .000000 .000000 .00000 SE B 0.000000 .000690 .000340 .000000 .000000 .00000 SSE B 0.000000 .000800 .000690 .000000 .000000 .00000 S B 0.000000 .002760 .001300 .000000 .000000 .00000 SSW B 0.000110 .003330 .003100 .000000 .000000 .00000 SW B 0.000110 .001380 .001490 .000000 .000000 .00000 WSW B 0.000110 .000570 .000800 .000000 .000000 .00000 W B 0.000000 .000340 .000460 .000000 .000000 .00000 WNW B 0.000000 .000230 .000460 .000000 .000000 .00000 NW B 0.000000 .000570 .001030 .000000 .000000 .00000 NNW 0.000110 .000570 .001030 .000000 .000000 .00000 N C 0.000570 .000800 .001490 .000230 .000000 .00000 NE C 0.000230 .005400 .002070 .000000 .000000 .00000 ENE C 0.000230 .002070 .002300 .000230 .000000 .00000 E C 0.000110 .000460 .000340 .000000 .000000 .00000 ESE C 0.000110 .000340 .000340 .000000 .000000 .00000 SE C 0.000000 .000230 .000800 .000000 .000000 .00000 SSE C 0.000110 .000800 .001030 .000110 .000000 .00000 s c 0.000110 .001030 .003330 .000690 .000000 .00000 SSW C 0.000110 .001380 .011030 .001260 .000000 .00000 SW C 0.000000 .000690 .006430 .000800 .000000 .00000 WSW C 0.000000 .000230 .001840 .000690 .000000 .00000 W C 0.000000 .000000 .001490 .000000 .000000 .00000 WNW C 0.000000 .000000 .000460 .000000 .000000 .00000 NW C 0.000000 .000230 .001150 .000110 .000000 .00000 NNW C 0.000110 .000340 .001380 .000340 .000000 .00000
N D 0.000690 .007930 .007700 .006890 .001260 .00000 NNE D 0.001950 .018040 .022750 .008160 .002640 .00218 NE D 0.000340 .021720 .031480 .011720 .002410 .00034 ENE D 0.000340 .009650 .009650 .002300 .000230 .00023 E D 0.000110 .001610 .001380 .000340 .000000 .00000 ESE D 0.000000 .000570 .001610 .000000 .000000 .00000 SE D 0.000110 .001490 .000800 .000340 .000110 .00000 SSE D 0.000570 .001490 .003680 .002410 .000340 .00011 S D 0.000570 .006550 .012060 .007240 .001610 .00046 SSW D 0.000690 .010110 .025390 .033550 .010800 .00172 SW D 0.000110 .007700 .028610 .045270 .029760 .01666 WSW D 0.000800 .002410 .008620 .011380 .007120 .00609 W D 0.000230 .001150 .003220 .001840 .000690 .00000 WNW D 0.000000 .000460 .001720 .000460 .000230 .00000 NW D 0.000110 .000340 .004830 .004370 .000460 .00011 NNW D 0.000230 .002760 .007240 .016200 .012640 .00184 N E 0.000700 .006780 .003100 .000000 .000000 .00000 NNE 0.002100 .009880 .006550 .000000 .000000 .00000 NE E 0.000700 .009310 .008960 .000000 .000000 .00000 ENE E 0.000350 .004480 .004250 .000000 .000000 .00000 E E 0.000120 .002300 .000920 .000000 .000000 .00000 ESE E 0.000120 .001260 .000460 .000000 .000000 .00000 SE E 0.000350 .001150 .000920 .000000 .000000 .00000 SSE E 0.000350 .001380 .001380 .000000 .000000 .00000 S E 0.000700 .004250 .003330 .000000 .000000 .00000 SSW E 0.001050 .007700 .008040 .000000 .000000 .00000 SW E 0.000470 .005970 .009770 .000000 .000000 .00000 WSW E 0.000700 .003680 .002760 .000000 .000000 .00000 W E 0.000350 .001840 .000690 .000000 .000000 .00000 WNW E 0.000000 .000690 .000460 .000000 .000000 .00000 NW E 0.000120 .000690 .001030 .000000 .000000 .00000 NNW E 0.000470 .002300 .000800 .000000 .000000 .00000 $N$ F 0.007720 .006890 .000000 .000000 .000000 .00000 NNE F 0.006320 .008730 .000000 .000000 .000000 .00000 NE E 0.005970 .012290 .000000 .000000 .000000 .00000 ENE F 0.005850 .006780 .000000 .000000 .000000 .00000 E F 0.003860 .006090 .000000 .000000 .000000 .00000 ESE F 0.003740 .002640 .000000 .000000 .000000 .00000 SE F 0.003280 .003560 .000000 .000000 .000000 .00000 SSE 0.004450 .005290 .000000 .000000 .000000 .00000 $S$ E 0.005850 .006430 .000000 .000000 .000000 .00000 SSW E 0.005030 .005400 .000000 .000000 .000000 .00000 SW E 0.004800 .007120 .000000 .000000 .000000 .00000 WSW F 0.003510 .005170 .000000 .000000 .000000 .00000

W F 0.003510 .003910 .000000 .000000 .000000 .00000 WNW F 0.003510 .003680 .000000 .000000 .000000 .00000 NW F 0.003860 .003100 .000000 .000000 .000000 .00000 NNW E 0.005030 .003220 .000000 .000000 .000000 .00000 
Table C-7. STAR File Used for PBF Ground Level Releases

N A 0.001620 .002860 .000000 .000000 .000000 .00000 NNE $A \quad 0.002540 .004230 .000000 .000000 .000000 .00000$ NE A 0.001040 .006520 .000000 .000000 .000000 .00000 ENE A 0.001960 .004690 .000000 .000000 .000000 .00000 E A 0.001160 .002170 .000000 .000000 .000000 .00000 ESE A 0.001160 .002170 .000000 .000000 .000000 .00000 SE A 0.000920 .001600 .000000 .000000 .000000 .00000 SSE A 0.000810 .003660 .000000 .000000 .000000 .00000 S A 0.001620 .004350 .000000 .000000 .000000 .00000 SSW A 0.001270 .004690 .000000 .000000 .000000 .00000 SW A 0.000810 .004460 .000000 .000000 .000000 .00000 WSW A 0.001620 .003320 .000000 .000000 .000000 .00000 W A 0.001270 .003430 .000000 .000000 .000000 .00000 WNW A 0.001040 .001600 .000000 .000000 .000000 .00000 NW A 0.001500 .001030 .000000 .000000 .000000 .00000 NNW A 0.001850 .002520 .000000 .000000 .000000 .00000 $N$ B 0.000110 .001370 .000570 .000000 .000000 .00000 NNE B 0.000340 .002970 .000230 .000000 .000000 .00000 NE B 0.000110 .002170 .000570 .000000 .000000 .00000 ENE B 0.000110 .001370 .001140 .000000 .000000 .00000 E B 0.000000 .001030 .000920 .000000 .000000 .00000 ESE B 0.000000 .000460 .000110 .000000 .000000 .00000 SE B 0.000000 .000570 .000340 .000000 .000000 .00000 SSE B 0.000000 .001030 .000800 .000000 .000000 .00000 S B 0.0001110 .002290 .002170 .000000 .000000 .00000 SSW B 0.000000 .003320 .002630 .000000 .000000 .00000 SW B 0.000230 .001720 .002630 .000000 .000000 .00000 WSW B 0.000000 .001030 .001600 .000000 .000000 .00000

W B 0.000000 .000340 .000800 .000000 .000000 .00000 WNW B 0.000000 .000460 .000570 .000000 .000000 .00000 NW B 0.000110 .000230 .000340 .000000 .000000 .00000 NNW B 0.000000 .000690 .000570 .000000 .000000 .00000 N C 0.000230 .000230 .001030 .000230 .000000 .00000 NNE C 0.000110 .003430 .001940 .000230 .000000 .00000 NE C 0.000230 .002860 .001600 .000000 .000000 .00000 ENE C 0.000000 .000800 .001600 .000110 .000000 .00000 E C 0.000000 .000570 .000570 .000000 .000000 .00000 ESE C 0.000000 .000230 .000340 .000000 .000000 .00000 SE C 0.000000 .000230 .000570 .000000 .000000 .00000 SSE C 0.000000 .000460 .002170 .000000 .000000 .00000 S C 0.000110 .001260 .004690 .000570 .000000 .00000 SSW C 0.000340 .001600 .009380 .001720 .000000 .00000 SW C 0.000000 .001140 .007660 .000920 .000000 .0000 WSW C 0.000000 .000460 .002860 .000570 .000000 .00000 W C 0.000230 .000800 .002170 .000340 .000000 .00000 WNW C 0.000230 .000000 .001140 .000110 .000000 .00000 NW C 0.000110 .000230 .000340 .000230 .000000 .00000 NNW C 0.000000 .000340 .000690 .000110 .000000 .00000
N D 0.000340 .002970 .004920 .006630 .004120 .00023 NNE D 0.001030 .021160 .024360 .015560 .004120 .00252 NE D 0.000340 .019900 .030420 .023220 .003430 .00114 ENE D 0.000000 .008920 .010290 .003660 .000000 .00000

E D 0.000000 .002290 .002520 .000570 .000000 .00000 ESE D 0.000000 .001140 .001370 .000340 .000000 .00000 SE D 0.000000 .000460 .002400 .003320 .000230 .00000 SSE D 0.000230 .004230 .012240 .008240 .001370 .00000 S D 0.000340 .006630 .015900 .011670 .002750 .00046 SSW D 0.000800 .006860 .017390 .020700 .009610 .00069 SW D 0.000460 .004580 .019900 .041860 .032250 .01544 WSW D 0.000460 .002170 .010980 .021730 .011210 .01281

W D 0.000230 .001490 .003320 .002860 .001140 .00011 WNW D 0.000000 .000800 .001940 .001600 .000000 .00011 NW D 0.000230 .000570 .001260 .000800 .000570 .00011 NNW D 0.000110 .001030 .002290 .004230 .003320 .00046 N E 0.000930 .002970 .000800 .000000 .000000 .00000 NNE E 0.001280 .007890 .005260 .000000 .000000 .00000 NE E 0.001040 .013380 .010750 .000000 .000000 .00000 ENE E 0.000230 .008460 .008810 .000000 .000000 .00000 E E 0.000120 .000920 .001720 .000000 .000000 .00000 ESE E 0.000000 .000920 .000690 .000000 .000000 .00000 SE E 0.000000 .001030 .001830 .000000 .000000 .00000 SSE E 0.000230 .002970 .005150 .000000 .000000 .00000 S E 0.001040 .006060 .004230 .000000 .000000 .00000 SSW E 0.001040 .005380 .004000 .000000 .000000 .00000 SW E 0.000810 .004000 .004120 .000000 .000000 .00000 WSW E 0.000460 .001490 .002630 .000000 .000000 .00000 W E 0.000230 .001030 .000230 .000000 .000000 .00000 WNW E 0.000120 .000570 .000110 .000000 .000000 .00000 NW E 0.000230 .001140 .000230 .000000 .000000 .00000 NNW E 0.000460 .001720 .000110 .000000 .000000 .00000 $N$ F 0.007660 .006060 .000000 .000000 .000000 .00000 ENE F 0.003250 .005830 .000000 .000000 .000000 .00000 E E 0.003250 .002970 .000000 .000000 .000000 .00000 ESE F 0.002670 .001720 .000000 .000000 .000000 .00000 SE F 0.003130 .004800 .000000 .000000 .000000 .00000 SSE E 0.003600 .004800 .000000 .000000 .000000 .00000 F 0.005340 .007210 .000000 .000000 .000000 .00000 SSW F 0.007080 .009490 .000000 .000000 .000000 .00000 SW F 0.007890 .005720 .000000 .000000 .000000 .00000 WSW F 0.006380 .003770 .000000 .000000 .000000 .00000 W E 0.005690 .003890 .000000 .000000 .000000 .00000 WNW F 0.005450 .004800 .000000 .000000 .000000 .00000

NW F 0.005450 .002400 .000000 .000000 .000000 .00000 NNW 0.006150 .005950 .000000 .000000 .000000 .00000 


\section{Table C-8. STAR File Used for TAN Ground Level Releases}

N A 0.002430 .003330 .000000 .000000 .000000 .00000 NNE A 0.003130 .003440 .000000 .000000 .000000 .00000 NE A 0.002090 .011020 .000000 .000000 .000000 .00000 .007920 .000000 .00000 E A 0.003820 .009760 .000000 .000000 .000000 .00000 ESE A 0.003710 .005390 .000000 .000000 .000000 .00000 SE A 0.003130 .008950 .000000 .000000 .000000 .00000 SSE A 0.002090 .005850 .000000 .000000 .000000 .00000 S A 0.001970 .007920 .000000 .000000 .000000 .00000 SSW A 0.002320 .004820 .000000 .000000 .000000 .00000 WSW A 0.001850 .001950 .000000 .000000 .000000 .00000 W A 0.000230 .001720 .000000 .000000 .000000 .00000 WNW A 0.001160 .001720 .000000 .000000 .000000 .00000 NW A 0.000810 .001490 .000000 .000000 .000000 .00000 NNW A 0.001620 .001840 .000000 .000000 .000000 .00000 N B 0.000000 .001150 .000920 .000000 .000000 .00000 NNE B 0.000460 .003440 .001380 .000000 .000000 .00000 NE B 0.001030 .004020 .000690 .000000 .000000 .00000 ENE B 0.000460 .003440 .000800 .000000 .000000 .00000 E B 0.000230 .002300 .001150 .000000 .000000 .00000 ESE B 0.000000 .001030 .000570 .000000 .000000 .00000 SE B 0.000230 .001490 .000690 .000000 .000000 .00000 SSE B 0.000000 .001260 .000460 .000000 .000000 .00000 S B 0.000000 .002750 .001490 .000000 .000000 .00000 SSW B 0.000340 .004130 .002640 .000000 .000000 .00000 SW B 0.000230 .001720 .002520 .000000 .000000 .00000 WSW B 0.000000 .000460 .001490 .000000 .000000 .00000 W B $0.000000,000000.000340 .000000 .000000 .00000$ WNW B 0.000000 .000230 .000000 .000000 .000000 .00000 NW B 0.000110 .000460 .000230 .000000 .000000 .00000 NWT B 0.000340 .000340 .000460 .000000 .000000 .00000 N C 0.000340 .001030 .002300 .000110 .000000 .00000 NNE C 0.000110 .002750 .001840 .000230 .000000 .00000 NE C 0.000460 .006890 .002640 .000110 .000000 .00000 ENE C 0.000340 .005050 .001150 .000110 .000000 .00000 E C 0.000460 .002520 .000920 .000110 .000000 .00000 ESE C 0.000110 .000340 .000460 .000110 .000000 .00000 SE C 0.000230 .001150 .000340 .000110 .000000 .00000 SSE C 0.000000 .000340 .000570 .000110 .000000 .00000 S C 0.000000 .001030 .003210 .000110 .000000 .00000 SSW C 0.000110 .001490 .010670 .000690 .000000 .00000 SW C 0.000110 .001030 .005740 .000460 .000000 .00000 WSW C 0.000340 .000110 .001380 .000690 .000000 .00000 W C 0.000000 .000000 .000800 .000460 .000000 .00000 WN C $0.000000,000000.001030 .000000 .000000 .00000$ NW C 0.000000 .000110 .000340 .000000 .000000 .00000 NNW C 0.000110 .000340 .001610 .000110 .000000 .00000
N D 0.001610 .037640 .016760 .008950 .003330 .00126 NNE D 0.001260 .027200 .018130 .005280 .001840 .00000 NE D 0.001150 .021690 .012400 TNE D 0.000460 .006430 .002300 .000110 .000110 .00000 E D 0.000110 .002300 .001030 .000570 .000000 .00000 ESE D 0.000340 .000920 .000570 .000110 .000000 .00000 SE D 0.000230 .000920 .000570 .000340 .000000 .00000 SSE D 0.000000 .001720 .001950 .000920 .000110 .00000 S D 0.000230 .002300 .004820 .002870 .000570 .00000 SSW D 0.000000 .004710 .014580 .012850 .001950 .00011 SW D 0.000920 .005740 .016300 .028120 .021690 .01056 WSW D 0.000690 .002870 .004940 .007690 .001610 .00126 W D 0.000000 .000460 .000690 .001030 .000000 .00000 WNW D 0.000000 .000000 .000570 .000340 .000000 .00000 NW D 0.000340 .001380 .003330 .002640 .000690 .00023 NNW D 0.000800 .020310 .017670 .024220 .028230 .01733 NE 0.001510 .023410 .004710 .000000 .000000 .00000 NNE 0.001510 .016180 .005740 .000000 .000000 .00000 NE E 0.001160 .006310 .002520 .000000 .000000 .00000 ENE E 0.000230 .002870 .000460 .000000 .000000 .00000 E E 0.000120 .000690 .000110 .000000 .000000 .00000 ESE E 0.000120 .000460 .000340 .000000 .000000 .00000 SE E 0.000350 .000340 .000230 .000000 .000000 .00000 SSE E 0.000120 .001490 .000800 .000000 .000000 .00000 S E 0.000120 .001380 .002300 .000000 .000000 .00000 SSW E 0.000350 .003900 .003330 .000000 .000000 .00000 SW E 0.000350 .003670 .003210 .000000 .000000 .00000 WSW E 0.000580 .001950 .000800 .000000 .000000 .00000 W E 0.000120 .000920 .000690 .000000 .000000 .00000 WNW E 0.000230 .000340 .000230 .000000 .000000 .00000 NW E 0.000580 .002300 .001610 .000000 .000000 .00000 NWW E 0.003020 .016640 .010560 .000000 .000000 .00000 1 0.008710 .018940 .010000 NNE F 0.008830 .019400 .000000 .000000 .000000 .00000 NE F 0.006390 .011130 .000000 .000000 .000000 .00000 ENE F $0.004300 .005390 .000000 .000000,000000.00000$ E F 0.002790 .002180 .000000 .000000 .000000 .00000 ESE F 0.001050 .003440 .000000 .000000 .000000 .00000 SE 0.001050 .001720 .000000 .000000 .000000 .00000 SE 0.001860 .002870 .000000 .000000 .000000 $S$ S 0.002090 .001840 .000000 .000000 .000000 .00000 S E 0.002090 .001840 .000000 .000000 .000000 .00000 SW F 0.003020 .007120 .000000 .000000 .000000 .00000 WSW F 0.003370 .003100 .000000 .000000 .000000 .00000 W F 0.005000 .004710 .000000 .000000 .000000 .00000 WW F 0.007200 .005510 .000000 .000000 .000000 .00000 NW F $0.006970 .006770 .000000 .000000 .000000,00000$ NNW F 0.009060 .012970 .000000 .000000 .000000 .00000 
Table C-9. STAR File Used for TAN Elevated Releases

N A 0.001390 .003100 .000340 .000000 .000000 .00000 NNE A $0.002320 .004130 .000110 .000000,000000.00000$ NE A 0.002900 .006770 .000110 .000000 .000000 .00000 ENE A 0.003020 .007000 .000000 .000000 .000000 .00000 E A 0.002090 .007570 .000340 .000000 .000000 .00000 ESE A 0.002790 .006200 .000000 .000000 .000000 .00000 SE A 0.002670 .006770 .000000 .000000 .000000 .00000 SSE A 0.002090 .007570 .000110 .000000 .000000 .00000 S A 0.001970 .006660 .000340 .000000 .000000 .00000 SSW A 0.000930 .004250 .000340 .000000 .000000 .00000 SW A 0.001740 .002180 .000000 .000000 .000000 .00000 WSW A 0.000700 .002410 .000230 .000000 .000000 .00000 W A 0.000810 .001720 .000000 .000000 .000000 .00000 WNW $A$ 0.001050.001490.000000.000000.000000.00000 NW A $0.000700 .001490 .000110: 000000.000000 .00000$ NNW A 0.001630 .001610 .000000 .000000 .000000 .00000 N B 0.000000 .000460 .001720 .000000 .000000 .00000 NNE B 0.000460 .002410 .001720 .000000 .000000 .00000 NE B 0.000230 .003790 .001380 .000000 .000000 .00000 ENE B 0.000230 .002980 .001150 .000000 .000000 .00000 E B 0.000230 .002300 .001380 .000000 .000000 .00000 ESE B 0.000230 .001150 .001260 .000000 .000000 .00000 SE B 0.000000 .000800 .001260 .000000 .000000 .00000 SSE B 0.000000 .000340 .000920 .000000 .000000 .00000 S B 0.000110 .001490 .001840 .000000 .000000 .00000 SSW B $0.000230,000800.004360 .000000 .000000 .00000$ SW B 0.000230 .000340 .002870 .000000 .000000 .00000 WSW B 0.000000 .000230 .001490 .000000 .000000 .00000 W B 0.000000 .000000 .000340 .000000 .000000 .00000 WNW B 0.000000 .000000 .000000 .000000 .000000 .00000 NW B 0.000110 .000110 .000800 .000000 .000000 .00000 NNW B 0.000000 .000340 .000460 .000000 .000000 .00000 N C 0.000230 .001150 .001840 .000460 .000000 .00000 NNE C 0.000230 .003790 .001380 .000570 .000000 .00000 NE C 0.000570 .006540 .003210 .000340 .000000 .00000 ENE C 0.000340 .004360 .001150 .000110 .000000 .00000 E C 0.000110 .002750 .001260 .000340 .000000 .00000 ESE C 0.000000 .000460 .000460 .000230 .000000 .00000 SE C 0.000230 .000460 .000570 .000110 .000000 .00000 SSE C 0.000110 .000690 .000340 .000230 .000000 .00000 S C 0.000230 .001490 .002410 .001150 .000000 .00000 SSW C 0.000110 .001380 .006540 .004130 .000110 .00000 SW C 0.000000 .001380 .003560 .001720 .000000 .00000 WSW C 0.000000 .000000 .000570 .001380 .000000 .00000 W C 0.000000 .000000 .000920 .000920 .000000 .00000 WNW C 0.000000 .000000 .000690 .000000 .000000 .00000 NW C 0.000000 .000340 .000690 .000230 .000000 .00000 NNW C 0.000000 .000110 .001150 .000000 .000000 .00000
N D 0.000700 .013200 .021920 .010560 .004360 .00161 NNE D 0.000590 .024100 .034550 .008610 .003210 .00080 NE D 0.000940 .019170 .017560 .005620 .001950 .00069 ENE D 0.000120 .006660 .003790 .000690 .000230 .00011 E D 0.000230 .002520 .001610 .000340 .000460 .00000 ESE D 0.000230 .000920 .000800 .000460 .000000 .00000 SE D 0.000230 .001030 .000800 .000570 .000230 .00000 SSE D 0.000000 .000460 .001380 .001610 .000340 .00011 S D 0.000000 .001380 .002870 .004020 .002300 .00080 SSW D 0.000590 .002640 .009300 .016070 .013770 .01366 SW D 0.000350 .002980 .005280 .017790 .017100 .02513 WSW D 0.000000 .000920 .001610 .006080 .003440 .00184 W D 0.000000 .000230 .000570 .001150 .000460 .00011 WNW D 0.000590 .000690 .001610 .000230 .000340 .00000 NW D 0.000820 .002180 .005620 .006540 .003330 .00172 NNW D 0.000230 .004480 .010100 .014690 .022720 .04247

$N$. 0.001050 .010330 .015040 .003560 .000000 .00000 NE E 0.000470 .006080 .007230 .002520 .000230 .00000 ENE E 0.000120 .001260 .002640 .000110 .000000 .00000 E E 0.000000 .001030 .001030 .000110 .000000 .00000 ESE E 0.000000 .000230 .000460 .000000 .000000 .00000 SE E 0.000230 .000230 .000800 .000340 .000000 .00000 SSE E 0.000120 .000340 .000690 .000920 .000000 .00000 $S$ E 0.000000 .000570 .001720 .001610 .000000 .00000 SSW E 0.000230 .000690 .002750 .007230 .001380 .00000 SW E 0.000470 .002180 .003210 .004480 .000110 .00000 WSW E 0.000350 .000570 .001030 .000920 .000110 .00000 W E 0.000000 .000570 .001150 .000000 .000000 .00000 WNW E 0.000230 .001030 .001030 .000000 .000000 .00000 NW E 0.001050 .001720 .002520 .001380 .000110 .00000 NNW E 0.000470 .003900 .007800 .005970 .000570 .00000 NNE F 0.003260 .013080 .008610 .000570 .000000 .00000 NE F 0.002450 .006770 .002070 .000000 .000000 .00000 ENE F 0.001980 .003440 .001260 .000000 .000000 .00000 E F 0.001750 .002070 .000800 .000000 .000000 .00000 ESE F 0.001870 .001840 .000230 .000000 .000000 .00000 SE F 0.000700 .001610 .000110 .000000 .000000 .00000 SSE F 0.001280 .001610 .000800 .000000 .000000 .00000 $S$ F 0.001520 .001150 .000570 .000110 .000000 .00000 SSW F 0.002210 .002410 .001380 .000340 .000000 .00000 SW F 0.001630 .005050 .001490 .000000 .000000 .00000 WSW F 0.001870 .003440 .000570 .000000 .000000 .00000 W E 0.002450 .003900 .000800 .000000 .000000 .00000 WNW F 0.002330 .003560 .000460 .000000 .000000 .00000 NW F 0.003730 .005620 .001380 .000110 .000000 .00000 NNW F 0.003730 .007920 .003100 .000230 .000000 .00000 


\section{Table C-10. STAR File Used for TRA Ground Level and Elevated Releases}

N A 0.000700 .001390 .000000 .000000 .000000 .00000 NNE A 0.002090 .002540 .000000 .000000 .000000 .00000 NE A 0.002210 .004620 .000000 .000000 .000000 .00000 A A 0.002210 .004620 .000000 .000000 .000000 .00000 E A 0.002320 .006590 .000000 .000000 .000000 .00000 ESE A 0.001860 .003700 .000000 .000000 .000000 .00000 SE A 0.001630 .002890 .000000 .000000 .000000 .00000 SSE A 0.001390 .004390 .000000 .000000 .000000 .00000 $S$ A 0.000810 .005320 .000000 .000000 .000000 .00000 SSW A 0.001050 .006710 .000000 .000000 .000000 .00000 SW A 0.001050 .003470 .000000 .000000 .000000 .00000 WSW A 0.000700 .000920 .000000 .000000 .000000 .00000 W A 0.000930 .000690 .000000 .000000 .000000 .00000 WNW A 0.000580 .001160 .000000 .000000 .000000 .00000 NW A 0.000930 .000350 .000000 .000000 .000000 .00000 NNW A 0.000700 .001160 .000000 .000000 .000000 .00000

N B 0.000120 .001040 .000350 .000000 .000000 .00000 NNE B 0.000810 .002200 .000350 .000000 .000000 .00000 NE $B \quad 0.000230 .002310 .000580 .000000 .000000 .00000$ ENE B 0.000460 .002430 .001160 .000000 .000000 .00000 E B 0.000350 .000920 .000350 .000000 .000000 .00000 ESE B 0.000000 .001160 .000460 .000000 .000000 .00000 SE B 0.000120 .000580 .000350 .000000 .000000 .00000 SSE B 0.000000 .002080 .001040 .000000 .000000 .00000 S B 0.000230 .002660 .001040 .000000 .000000 .00000 ST B 0.000230 .002650 .001040 .000000 .000000 .00000 SW B 0.000000 .002310 .003010 .000000 .000000 .00000 WSW B 0.000120 .000920 .001500 .000000 .000000 .00000 W B 0.000000 .000350 .000810 .000000 .000000 .00000 WNW B 0.000120 .000580 .000230 .000000 .000000 .00000 NW B 0.000000 .000120 .000230 .000000 .000000 .00000 NNW B 0.000000 .000230 .000120 .000000 .000000 .00000 N C 0.000120 .000580 .001270 .000120 .000000 .00000 NNE C 0.000230 .001850 .001970 .000460 .000000 .00000 NE C 0.000230 .003350 .003120 .000230 .000000 .00000 ENE C 0.000000 .002540 .002430 .000230 .000000 .00000 E C 0.000350 .000690 .000580 .000120 .000000 .00000 ESE C 0.000230 .000120 .000350 .000000 .000000 .00000 SE C 0.000120 .000350 .000690 .000000 .000000 .00000 SSE C 0.000000 .000350 .000920 .000120 .000000 .00000 s 0.000120 .001160 .002080 .000230 .000000 .00000 SSW C 0.000000 .001620 .009830 .000810 .000000 .00000 SW C 0.000000 .001270 .007400 .001270 .000000 .00000 WSW C 0.000000 .000460 .003470 .000690 .000000 .00000 W C 0.000000 .000350 .001970 .000000 .000000 .00000 WNW C 0.000000 .000000 .001160 .000000 .000000 .00000 NW C 0.000000 .000120 .000920 .000230 .000000 .00000 NNW C 0.000000 .000230 .001500 .000120 .000000 .00000
N D 0.000690 .009830 .005660 .006940 .004050 .00058 NNE D 0.001270 .016650 .019540 .007750 .002080 .00197 NE D 0.001620 .022890 .032600 .016180 .001850 .00081 ENE D 0.000460 .004390 .006940 .002660 .000460 .00023 E D 0.000120 .001500 .001160 .000230 .000000 .00000 ESE D 0.000000 .000460 .000810 .000230 .000000 .00000 SE D 0.000000 .001040 .000810 .000690 .000000 .00000 SSE D 0.000120 .002660 .003700 .001970 .000120 .00012 $S$ D 0.000580 .004510 .009130 .003930 .001500 .00035 SW D 0.000230 .009020 .040460 .065550 .030060 .01584 WSW D 0.000460 .006240 .020690 .019420 .010520 .00890 W D 0.000460 .003350 .004860 .004160 .001390 .00012 WNW D 0.000350 .001160 .002770 .001850 .000920 .00000 NW D 0.000460 .001500 .001850 .001390 .000580 .00035 NNW D 0.000120 .002080 .002660 .002430 .001500 .00023 N E 0.001520 .007860 .002660 .000000 .000000 .00000 NNE E 0.001520 .013070 .002660 .000000 .000000 .00000 NE E 0.001170 .008320 .010290 .000000 .000000 .00000 ENE E 0.000120 .002310 .004280 .000000 .000000 .00000 E E 0.000000 .001160 .000690 .000000 .000000 .00000 ESE E 0.000000 .000920 .000460 .000000 .000000 .00000 SE E $0.000000 .000920 .000580,000000.000000 .00000$ SSE E 0.000120 .001970 .002080 .000000 .000000 .00000 $S$ E 0.000120 .004050 .003010 .000000 .000000 .00000 SW E 0.001050 .005200 .011560 .000000 .000000 .00000 WSW E 0.001170 .004740 .007400 .000000 .000000 .00000 W E 0.000120 .003350 .001160 .000000 .000000 .00000 WNW E 0.000590 .001040 .000580 .000000 .000000 .00000 NW E 0.000590 .001970 .000580 .000000 .000000 .00000 NNW E 0.000590 .001970 .000350 .000000 .000000 .00000 $N$ F 0.007010 .007050 .000000 .000000 .000000 .00000 NNE F 0.007830 .011210 .000000 .000000 .000000 .00000 NE F 0.005030 .005780 .000000 .000000 .000000 .00000 ENE F 0.003740 .004050 .000000 .000000 .000000 .00000 E F 0.003160 .002770 .000000 .000000 .000000 .00000 ESE F 0.002100 .002770 .000000 .000000 .000000 .00000 SE F 0.002460 .002890 .000000 .000000 .000000 .00000 SSE $F 0.003160 .003700 .000000 .000000 .000000 .00000$ $S$ F 0.002690 .006360 .000000 .000000 .000000 .00000 SSW F 0.004910 .006820 .000000 .000000 .000000 .00000 SW F 0.004560 .007750 .000000 .000000 .000000 .00000 WSW F 0.003970 .007400 .000000 .000000 .000000 .00000 W F 0.005030 .005200 .000000 .000000 .000000 .00000 WNW F 0.004910 .005090 .000000 .000000 .000000 .00000 NNW F 0.006200 .006590 .000000 .000000 .000000 .000000 


\section{APPENDIX D}

Input Parameter Values

For CAP-88 Computer Code 


\section{Table D-1. Input Parameter Values For CAP-88 Computer Code}

\begin{tabular}{|c|c|c|}
\hline RADIONUCLIDE-INDEPENDENT PARAMETERS & VALUE & REFERENCE \\
\hline TIME DELAY-INGESTION OF LEAFY VEGETABLES BY MAN (HR) & 24.0 & NRC (1977) \\
\hline TIME DELAY-INGESTION OF PRODUCE BY MAN (HR) & $1,440.0$ & NRC (1977) \\
\hline \multicolumn{3}{|l|}{ AGRICULTURAL PRODUCTIVITY BY UNIT AREA } \\
\hline (PRODUCE OR LEAFY VEG INGESTED BY MAN (KG/SQ METER)) & 2.0 & NRC (1977) \\
\hline RATE OF INGESTION OF PRODUCE BY MAN (KG/YR) & 520.0 & NRC (1977) \\
\hline RATE OF INGESTION OF MILK BY MAN (LITERS/YR) & 310.0 & NRC (1977) \\
\hline RATE OF INGESTION OF MEAT BY MAN (KG/YR) & 110.0 & NRC (1977) \\
\hline RATE OF INGESTION OF LEAFY VEGETABLES BY MAN (KG/YR) & 64.0 & NRC (1977) \\
\hline PERIOD OF LONG-TERM BUILDUP FOR ACTIVITY IN SOIL (YEARS) & 15.0 & NRC (1977) \\
\hline \multicolumn{3}{|l|}{ EFFECTIVE SURFACE DENSITY OF SOIL (KG/SQ. M), DRY WEIGHT. } \\
\hline (ASSUMES 15-CM PLOW LAYER) & 225.0 & DOE (1987) \\
\hline \multicolumn{3}{|l|}{ FRACIION OF RADIOACTIVITY RETAINED ON LEAFY } \\
\hline VEGETABLES AND PRODUCE AFTER WASHING & 1.0 & DOE (1987) \\
\hline \multicolumn{3}{|l|}{ SITE INFORMATION } \\
\hline HEIGHT OF LID & $800(\mathrm{M})$ & \\
\hline RAINFALL RATE & $21.7(C M / Y)$ & \\
\hline AVERAGE AIR TEMPERATURE & 5.8 (DEG C) & $279.0(\mathrm{~K})]$ \\
\hline SURFACE ROUGHNESS LENGTH (Z0) & $0.010(\mathrm{M})$ & \\
\hline
\end{tabular}

\begin{tabular}{|c|c|c|c|c|}
\hline & LOCAL & REGIONAL & IMPORTED & REFERENCF \\
\hline VEGETABLE: & 0.700 & 0.000 & 0.300 & DOE (198 \\
\hline MEAT: & 0.442 & 0.000 & 0.558 & DOE (1987) \\
\hline MILK: & 0.399 & 0.000 & 0.601 & DOE (1987) \\
\hline
\end{tabular}

Note: Variables not listed here are assigned the default values for CAP-88.

DEPOSITION VELOCITIES, SCAVENGING COEFFICIENTS, AND SEITLING VELOCITIES

\begin{tabular}{lcl} 
CATEGORY & $\begin{array}{c}\text { DEPOSITION } \\
\text { VELOCITY } \\
(\mathrm{m} / \mathrm{s})\end{array}$ \\
\cline { 1 - 1 } Noble gases & $\frac{0.0}{\text { Reactive gases }}$ & 0.035 \\
Organic iodine & 0.00018 \\
Particulates & 0.0018
\end{tabular}




\section{REFERENCES}

DOE (U.S. Department of Energy), 1987, Environmental Assessment: Fuel Processing Restoration at the Idaho National Engineering Laboratory, DOE/EA-0306.

EPA (U.S. Environmental Protection Agency), 1989, Risk Assessments Methodology, Environmental Impact Statement, NESHAPS for Radiomuclides, Background Information Document - Volume 1, EPA/520/1-89-005.

ICRP (International Commission on Radiological Protection), 1975, International Commission on Radiological Protection, Task Group Report on Reference Man, ICRP Publication 23, Pergamon Press, NY.

NRC (U.S. Nuclear Regulatory Commission), 1977, Regulatory Guide 1.109 Calculation of Anmual Doses to Man From Routine Releases of Reactor Effluents for the Purpose of Evaluating Compliance With 10 CFR Part 50 Appendix I, Revision 1. 
APPENDIXE

Supplemental Information 


\section{SUPPLEMENTAL INFORMATION}

The following information is provided at the request of DOE Headquarters and is not required as part of the annual NESHAPs reporting requirements.

REQUEST: Provide an estimate of collective effective dose equivalent (person-rem/yr) for 1997 releases.

An estimate of collective effective dose equivalent (person-rem/yr) is provided in the Idaho National Engineering and Environmental Laboratory Site Environmental Report for Calendar Year 1997 (DOE/DD-12082(97)). This collective EDE is calculated using the Mesoscale Diffussion (MDIFF) model and not CAP-88.

REQUEST: Provide information on the status of compliance with subparts $Q$ and $T$ of 40 CFR Part 61 if pertinent.

Subparts $Q$ and T of 40 CFR 61 are not pertinent to the INEEL.

REQUEST: Although exempt from Subpart H, provide information on Rn-220 emission from sources containing U-232 and Th-232 where emissions potentially can exceed 0.1 mrem/yr to the public or $10 \%$ of the nonradon dose to the public.

Not applicable at the INEEL.

REQUEST: Provide information on nondisposal/nonstorage sources of $\mathbf{R n}-222$ emissions where emissions potentially can exceed $0.1 \mathrm{mrem} / \mathrm{yr}$ to the public or $10 \%$ of the nonradon dose to the public.

Not applicable at the INEEL.

REQUEST: For the purpose of assessing facility compliance with the NESHAPs effluent monitoring requirements of Subpart $\mathrm{H}$ under Section 61.93(b), give the number of emission points subject to the continuous monitoring requirement, the number of these emission points that do not comply with the Section $61.93(b)$ requirements, 
and if possible, the cost for upgrades. Describe site periodic confirmatory measurement plans. Indicate the status of the QA program described by Appendix B, Method 114.

During CY 1997, the INEEL had six emission points that were subject to continuous compliance monitoring requirements as required by NESHAPs. All Six emission points comply with 40 CFR 61.93(b) requirements during normal operations.

DOE-ID has implemented an INEEL Periodic Confirmatory Measurements Program. The guidance was issued in 1992. Radiological atmospheric release points were divided into categories for implementing confirmatory measurements. Those emission points with a potential unabated emission greater than or equal to $0.1 \mathrm{mrem} /$ year require continuous compliance monitoring per 40 CFR 61.93. Periodic confirmatory measurements in the form of annual grab samples are required for emission sources where unabated emissions could result in an EDE between 0.01 and $0.1 \mathrm{mrem} / \mathrm{year}$. For an estimated EDE of less than $0.01 \mathrm{mrem} /$ year for a release point, as a minimum the source must be evaluated annually based upon process knowledge and the last 12 months of operation.

In 1997, each contractor performed the required periodic confirmatory measurements. Each vent was characterized and evaluated through continuous monitoring, grab sampling, or process knowledge. The results of the confirmatory measurements are on file with each contractor. Six radiological release points at the INEEL require continuous monitoring, approximately 45 additional release points were confirmed with either grab sampling or continuous sampling (not required by 40 CFR 61.93), and the remaining release points were confirmed through process knowledge and the last 12 months of operation. The New Waste Calcining Facility (NWCF) cell ventilation stack was redesigned this year (1997) to be a continuous compliance monitored stack.

Quality Assurance requirements specific to NESHAPs are specified in the INEEL Environmental Monitoring Plan. As a minimum, each INEEL facility with an emission potential of 0.1 $\mathrm{mrem} / \mathrm{yr}$ (assuming no allowance for pollution control equipment) is required to implement the NESHAPs QA requirements. To verify compliance, contractor QA plans have been reviewed against the NESHAPs QA requirements. 